\title{
PARFUME Theory and Model Basis Report
}

G. K. Miller

D. A. Petti

J. T. Maki

D. L. Knudson

September 2009
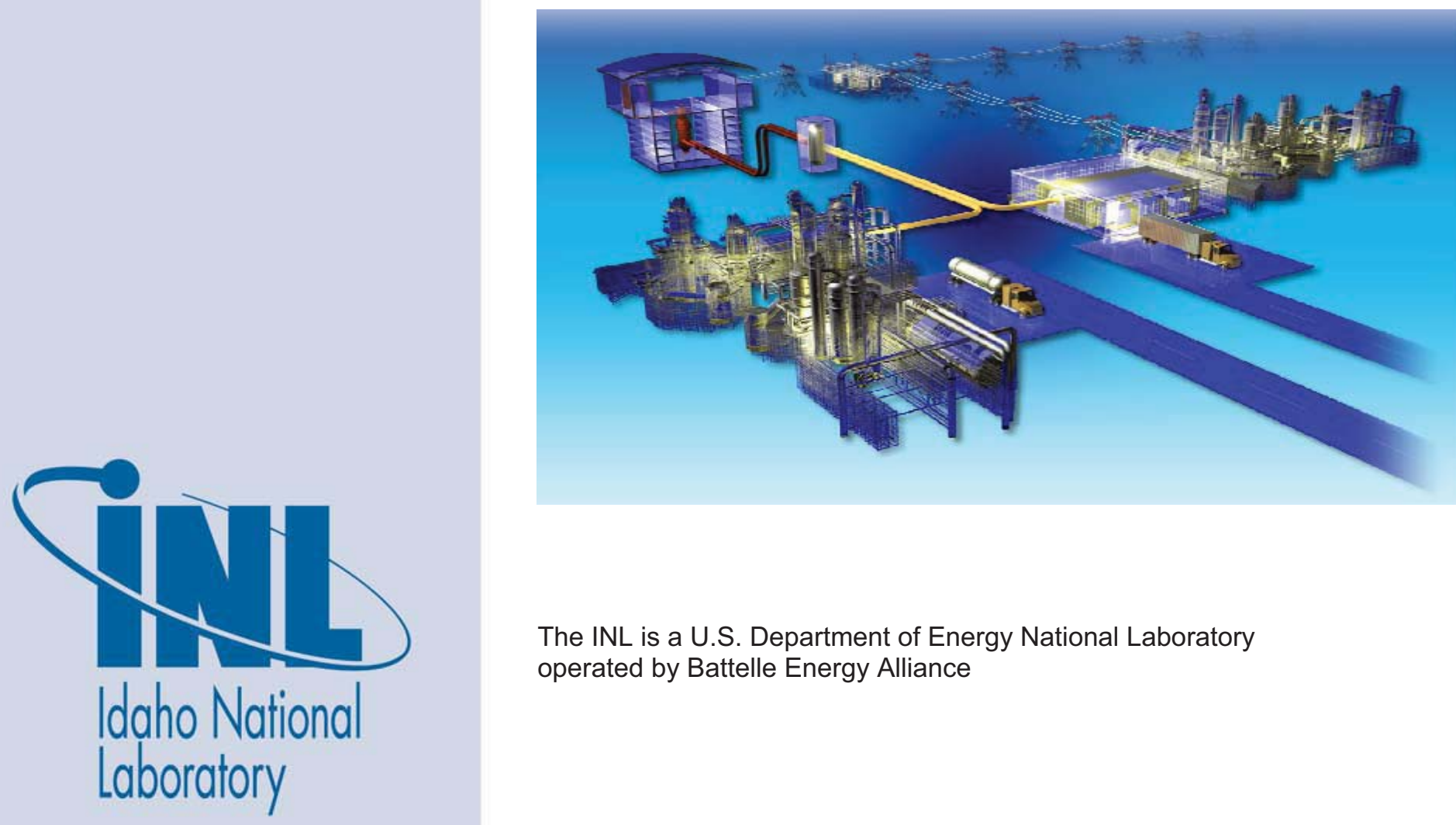

The INL is a U.S. Department of Energy National Laboratory operated by Battelle Energy Alliance 
INL/EXT-08-14497

\title{
PARFUME Theory and Model Basis Report
}

\author{
G. K. Miller \\ D. A. Petti \\ J. T. Maki \\ D. L. Knudson
}

September 2009

\section{Idaho National Laboratory \\ Next Generation Nuclear Plant Project \\ Idaho Falls, Idaho 83415}

http://www.inl.gov

Prepared for the

U.S. Department of Energy

Office of Nuclear Energy

Under DOE Idaho Operations Office

Contract DE-AC07-05ID14517 


\section{DISCLAIMER}

This information was prepared as an account of work sponsored by an agency of the U.S. Government. Neither the U.S. Government nor any agency thereof, nor any of their employees, makes any warranty, express or implied, or assumes any legal liability or responsibility for the accuracy, completeness, or usefulness of any information, apparatus, product, or process disclosed, or represents that its use would not infringe privately owned rights. References herein to any specific commercial product, process, or service by trade name, trademark, manufacturer, or otherwise, does not necessarily constitute or imply its endorsement, recommendation, or favoring by the U.S. Government or any agency thereof. The views and opinions of authors expressed herein do not necessarily state or reflect those of the U.S. Government or any agency thereof. 

Next Generation Nuclear Plant Project

PARFUME Theory and Model Basis Report

INL/EXT-08-14497

September 2009

Approved by:

John I. Mali

J. T. Maki

Principal Investigator

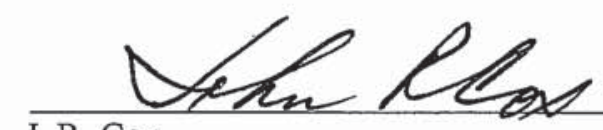

J. R. Cox

NGNP Deputy Director

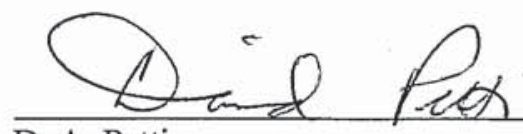

D. A. Patti NGNP Director

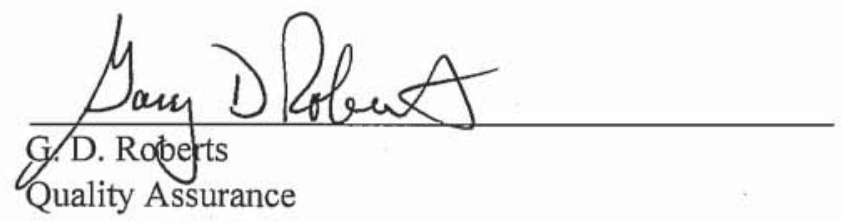

$9-10-09$

Date

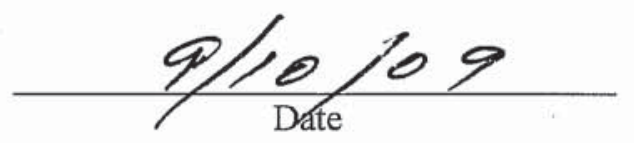

$\frac{9-10-09}{\text { Date }}$

$\frac{9 / 10 / 2009}{\text { Date }}$ 



\begin{abstract}
The success of gas reactors depends upon the safety and quality of the coated particle fuel. The fuel performance modeling code PARFUME simulates the mechanical, thermal and physico-chemical behavior of fuel particles during irradiation. This report documents the theory and material properties behind various capabilities of the code, which include: 1) various options for calculating CO production and fission product gas release, 2) an analytical solution for stresses in the coating layers that accounts for irradiation-induced creep and swelling of the pyrocarbon layers, 3) a thermal model that calculates a time-dependent temperature profile through a pebble bed sphere or a prismatic block core, as well as through the layers of each analyzed particle, 4) simulation of multi-dimensional particle behavior associated with cracking in the IPyC layer, partial debonding of the IPyC from the SiC, particle asphericity, and kernel migration (or amoeba effect), 5) two independent methods for determining particle failure probabilities, 6) a model for calculating release-to-birth ratios of gaseous fission products that accounts for particle failures and uranium contamination in the fuel matrix, and 7) the evaluation of an accident condition, where a particle experiences a sudden change in temperature following a period of normal irradiation. The accident condition entails diffusion of fission products through the particle coating layers and through the fuel matrix to the coolant boundary. This document represents the initial version of the PARFUME Theory and Model Basis Report. More detailed descriptions will be provided in future revisions.
\end{abstract}




\section{CONTENTS}

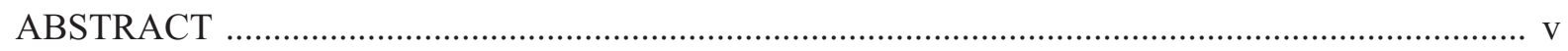

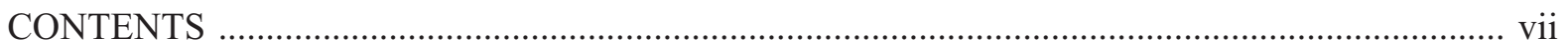

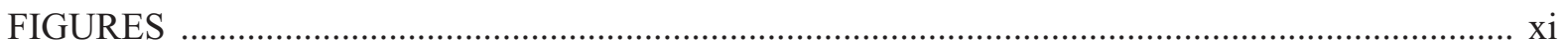

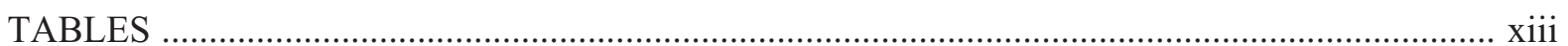

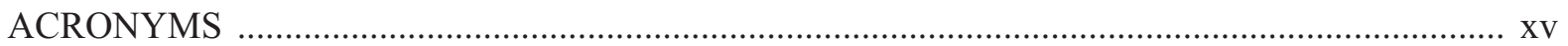

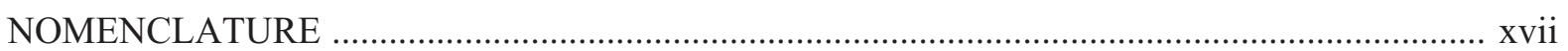

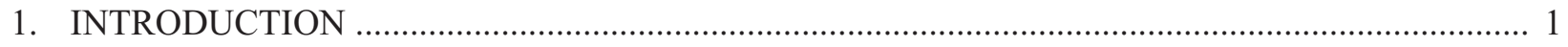

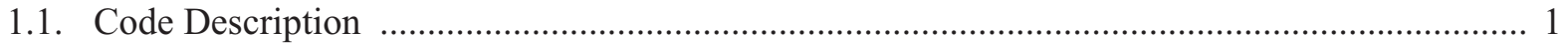

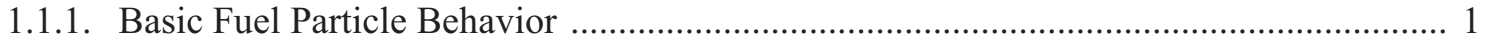

1.1.2. Basic Fuel Element Behavior ................................................................................... 3

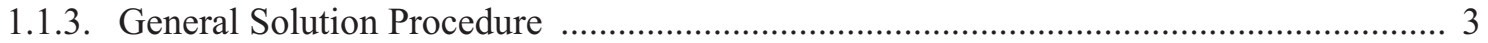

1.1.3.1. Compute Fuel Element "Macro" Temperature Profile ....................................... 3

1.1.3.2. Compute Fuel Particle "Micro" Temperature Profile ......................................... 5

1.1.3.3. Compute Particle Stress Distribution ................................................................ 5

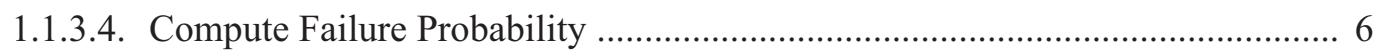

1.1.3.5. Compute Fission Product Diffusion ................................................................ 6

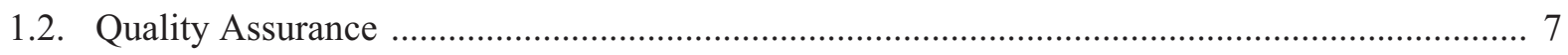

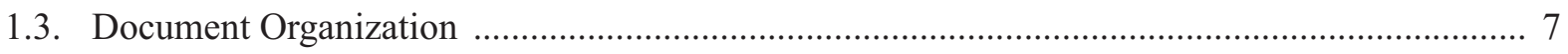

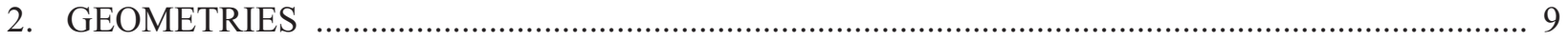

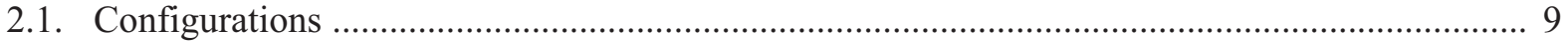

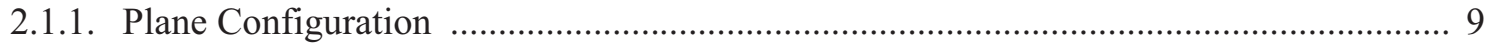

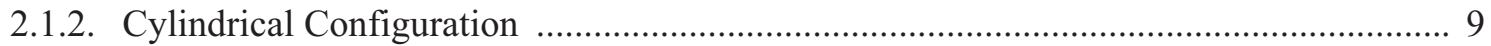

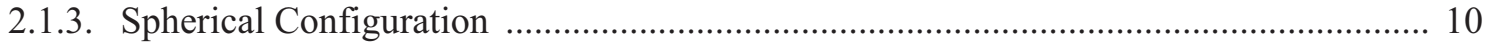

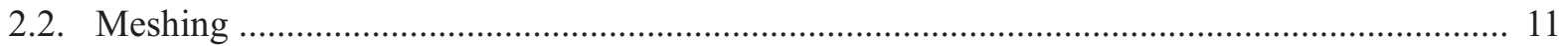

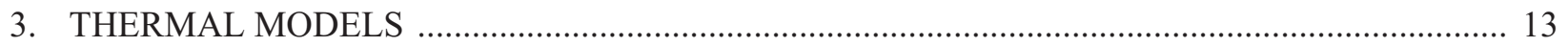

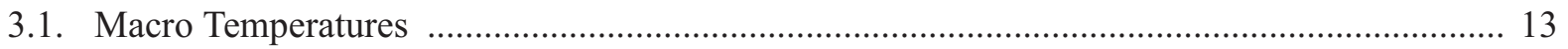

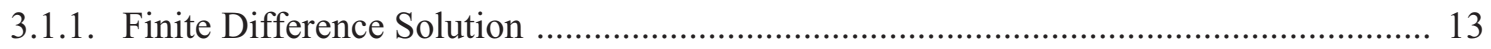

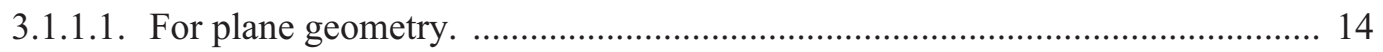

3.1.1.2. For cylindrical geometry. ........................................................................ 15

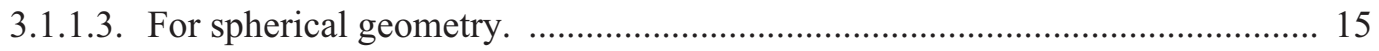

3.1.2. Macro Temperature Initialization …................................................................... 16

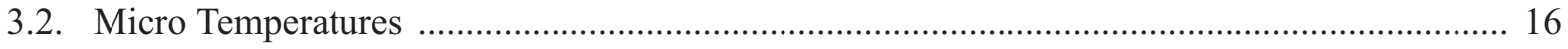




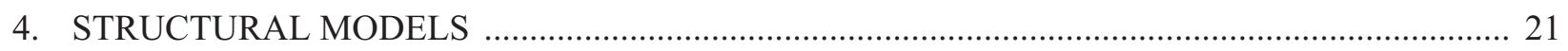

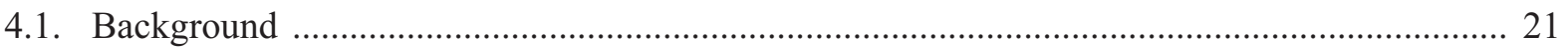

4.2. Stress Distribution Theory and Derivation ............................................................................ 22

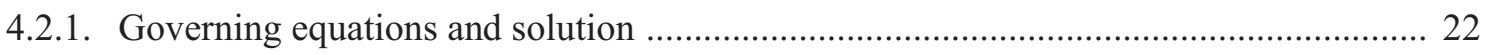

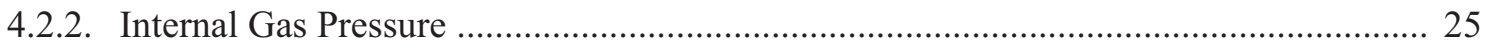

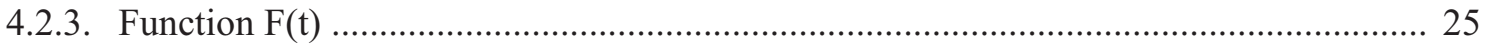

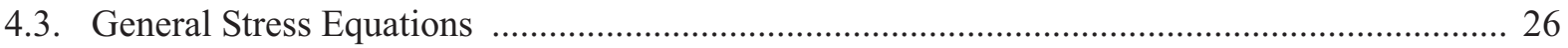

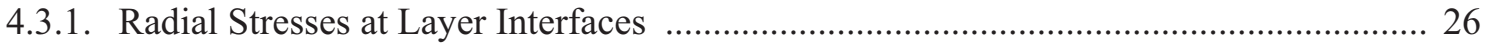

4.3.2. General Equations for Radial and Tangential Stresses .............................................. 28

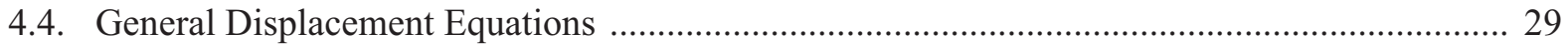

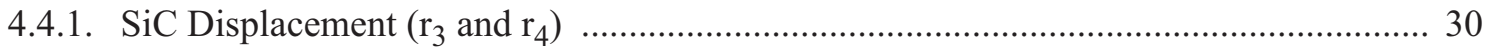

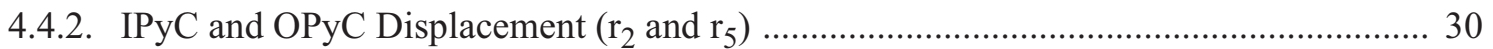

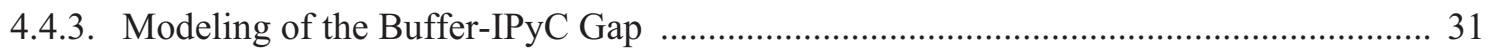

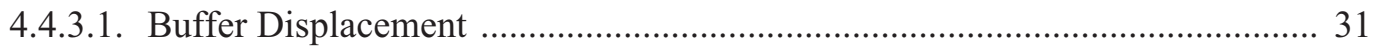

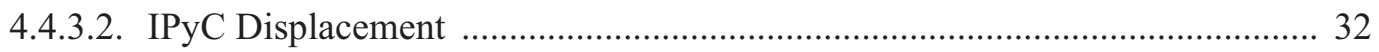

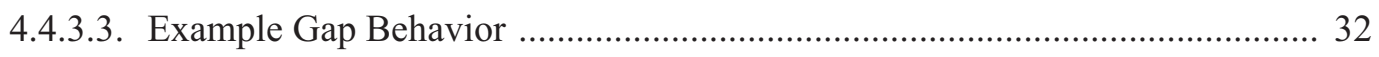

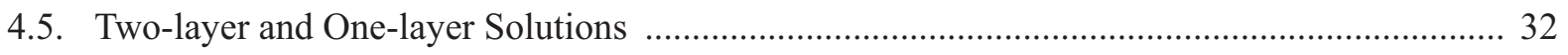

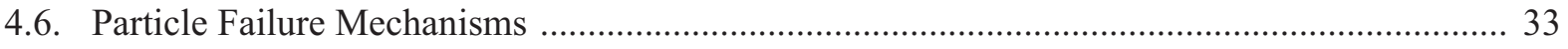

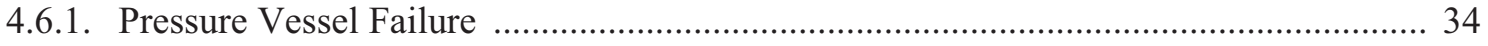

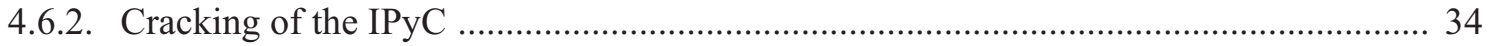

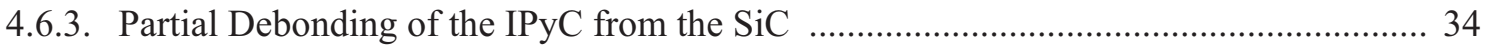

4.6.4. Pressure Vessel Failure of an Aspherical Particle ....................................................... 36

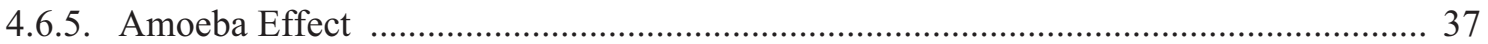

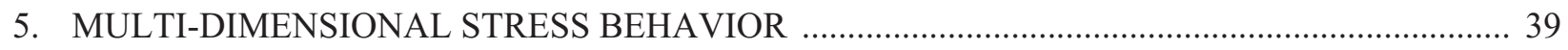

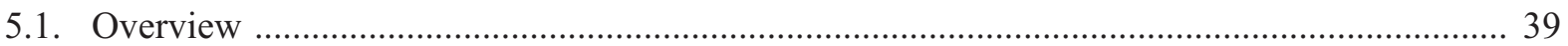

5.2. Statistical Approach for Determining Stresses .................................................................... 39

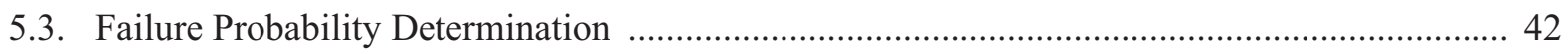

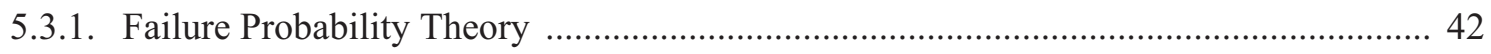

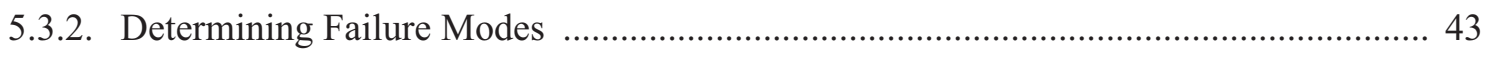

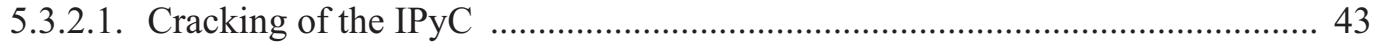

5.3.2.2. Partial Debonding of the IPyC from the $\mathrm{SiC}$................................................ 44

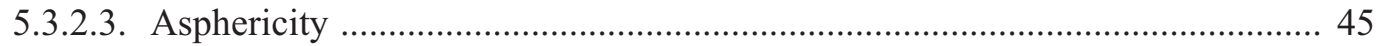

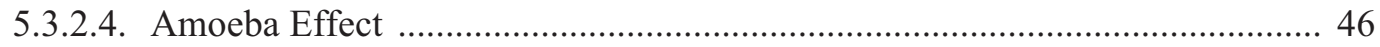

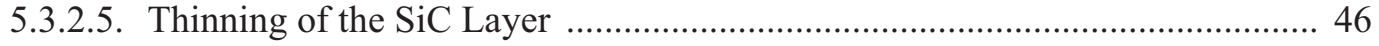


5.4. Solution Schemes for Predicting Failure ............................................................................ 47

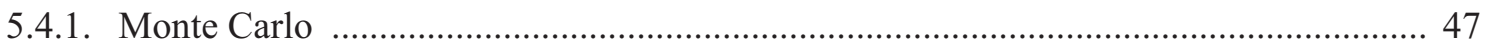

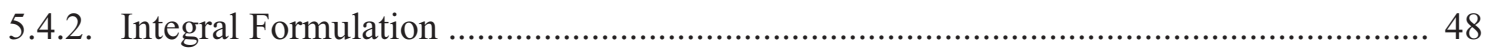

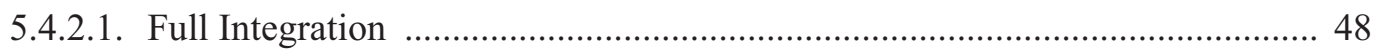

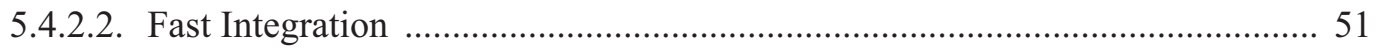

5.5. Resolution of Failure Probabilities in the Integration Method .............................................. 55

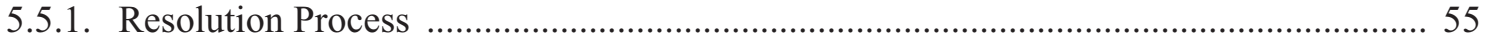

5.5.2. Accumulation of Failure Probabilities Through Time ................................................ 57

5.6. Linking Particle Failures with the Diffusion Calculations .................................................... 58

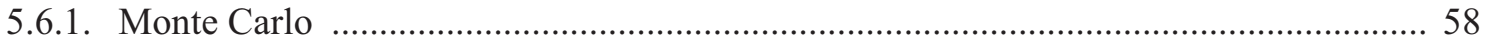

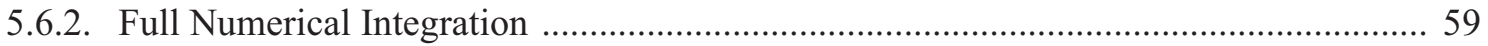

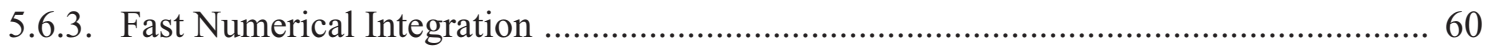

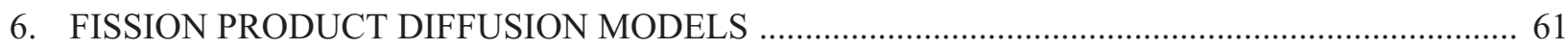

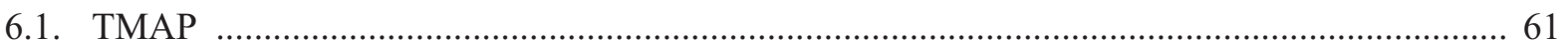

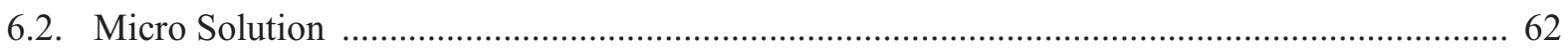

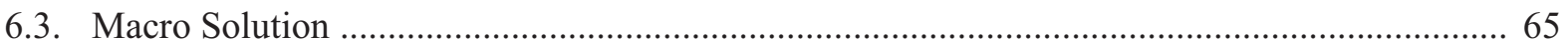

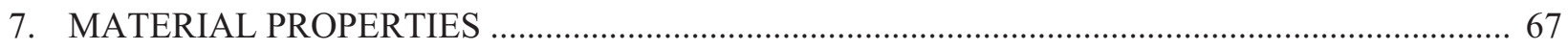

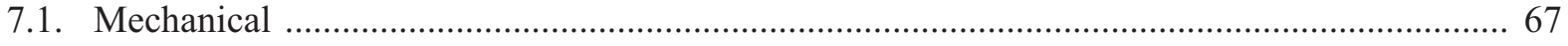

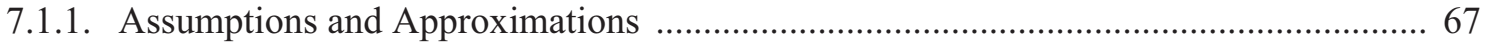

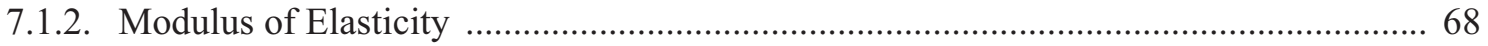

7.1.2.1. Pyrocarbons (IPyC and OPyC layers) ............................................................ 68

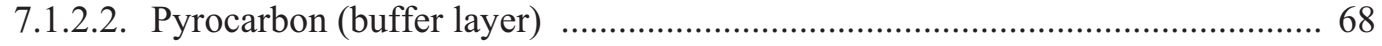

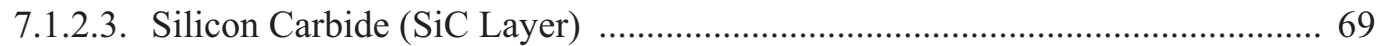

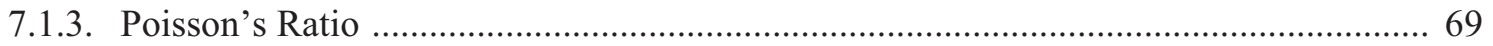

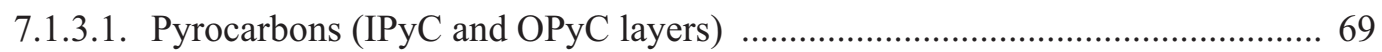

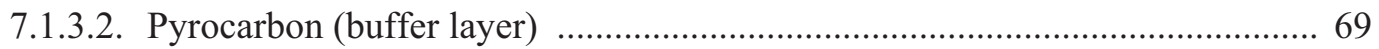

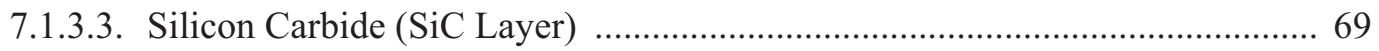

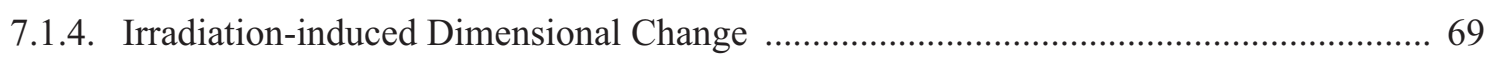

7.1.4.1. Pyrocarbons (IPyC and OPyC layers) .......................................................... 69

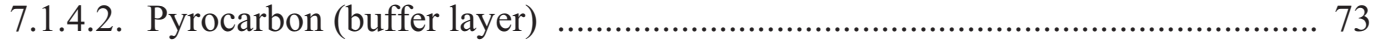

7.1.4.3. Silicon Carbide ( $\mathrm{SiC}$ Layer) ……................................................................. 74

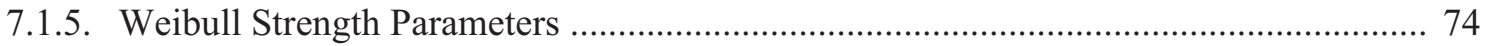

7.1.5.1. Pyrocarbons (IPyC and OPyC layers) ........................................................... 74

7.1.5.2. Pyrocarbon (buffer layer) …….................................................................. 75 
7.1.5.3. Silicon Carbide (SiC Layer) …………...................................................... 75

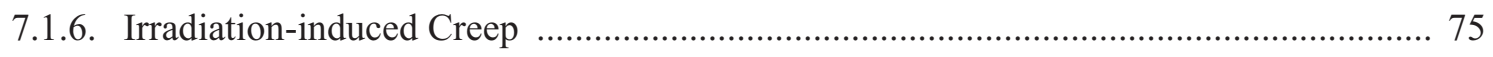

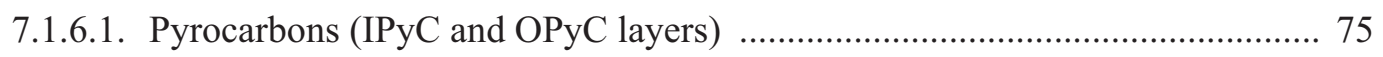

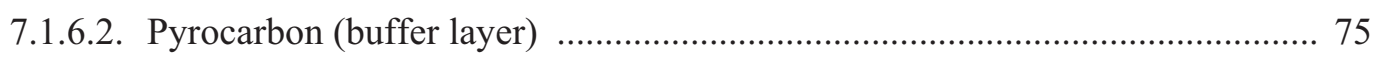

7.1.6.3. Silicon Carbide (SiC Layer) ………........................................................ 75

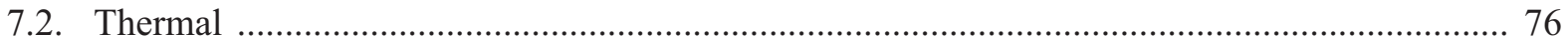

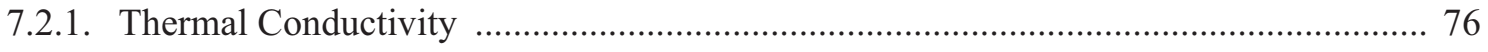

7.2.1.1. Pyrocarbons (IPyC and OPyC layers) ……................................................... 76

7.2.1.2. Pyrocarbon (buffer layer) ………….............................................................. 76

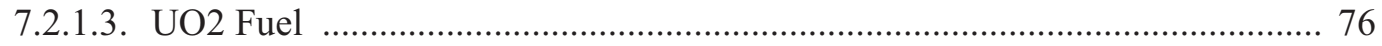

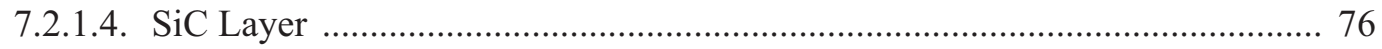

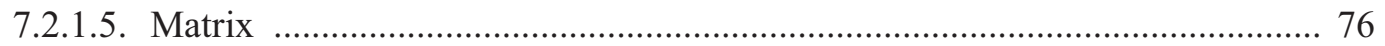

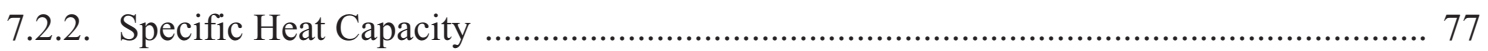

7.2.2.1. Pyrocarbons (IPyC and OPyC layers) …….................................................. 77

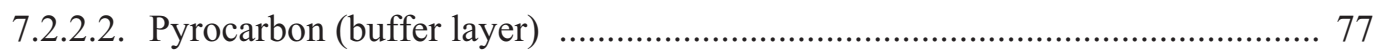

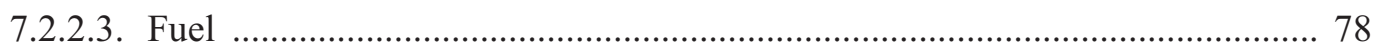

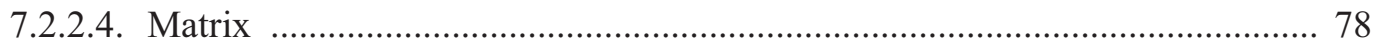

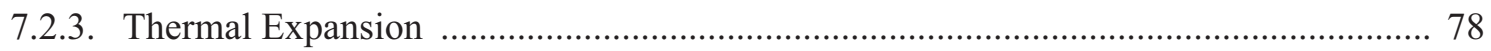

7.2.3.1. Pyrocarbons (IPyC and OPyC layers) …….................................................. 78

7.2.3.2. Pyrocarbon (buffer layer) ……………...................................................... 79

7.2.3.3. Silicon Carbide (SiC Layer) …………........................................................ 79

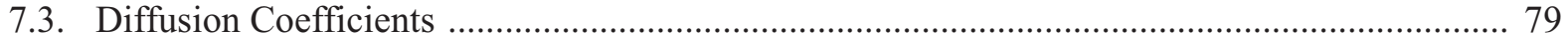

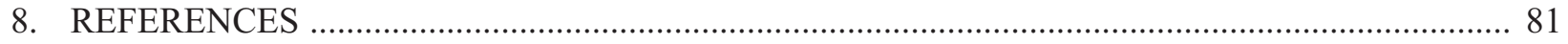

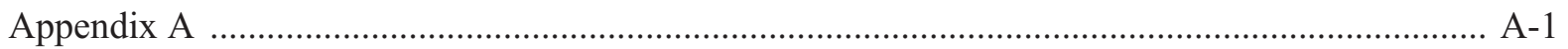




\section{FIGURES}

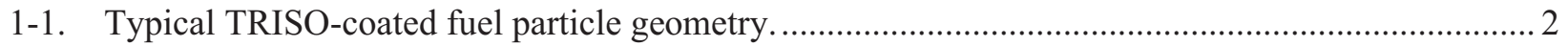

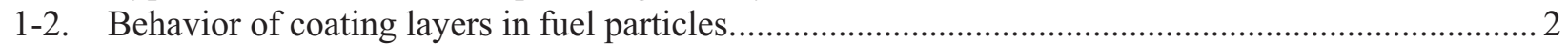

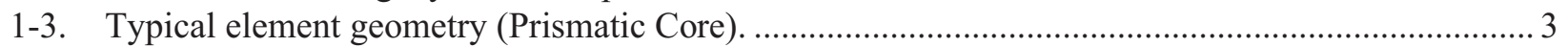

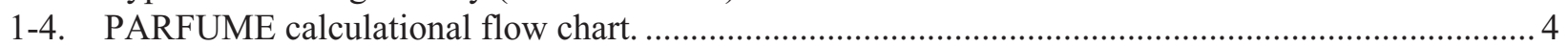

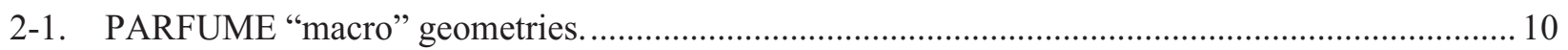

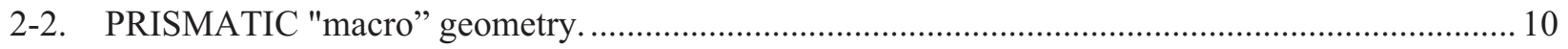

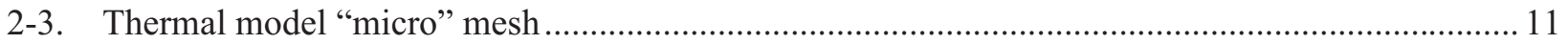

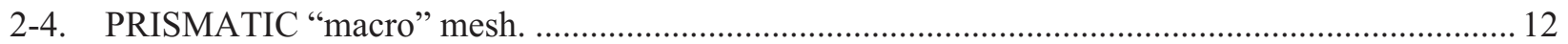

3-1. Finite difference notation used in the macro thermal model......................................................... 14

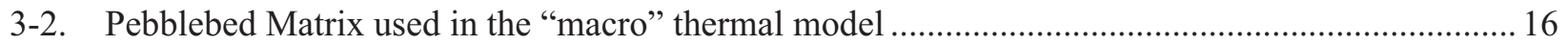

3-3. Fuel particle notation used in the "micro" thermal model. ......................................................... 18

3-4. Kernel centerline temperatures as functions of particle power and burnup...................................2 20

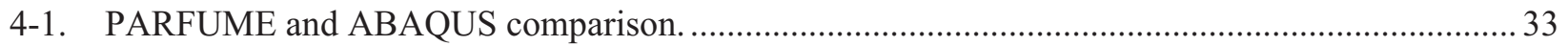

4-2. Finite element model for fuel particle having radial crack in IPyC layer........................................ 35

4-3. Stress history in $\mathrm{SiC}$ layer (near crack tip) for cracked particle. .................................................. 35

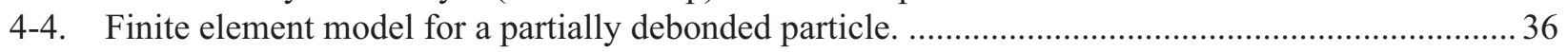

4-5. Inner $\mathrm{SiC}$ layer stress histories at two points that experience debonding...................................... 37

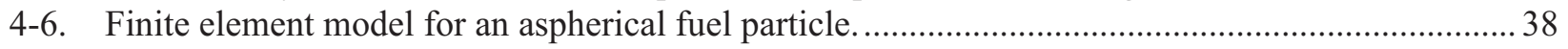

4-7. Stress histories for a faceted and spherical fuel particle. ..................................................... 38

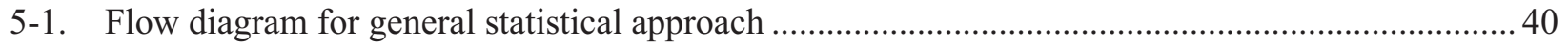

5-2. Generating an $\mathrm{h}$ function for variations in IPyC density .............................................................. 41

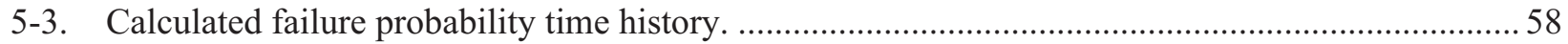

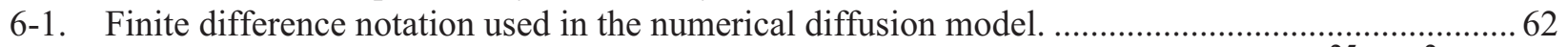

7-1. Irradiation Strain vs. Preirradiation Density for PyC irradiated at $1100{ }^{\circ} \mathrm{C}$ to $3.7 \times 10^{25} \mathrm{n} / \mathrm{m}^{2} \ldots \ldots . .73$ 


\section{TABLES}

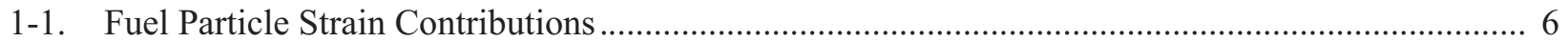

5-1. Number of integration points required for the three integration methods. .................................. 54

7-1. SiC Modulus of Elasticity as a Function of Temperature ............................................................. 69

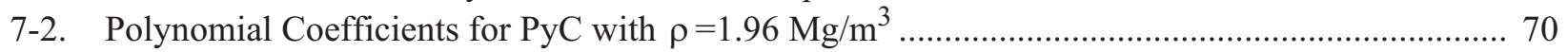

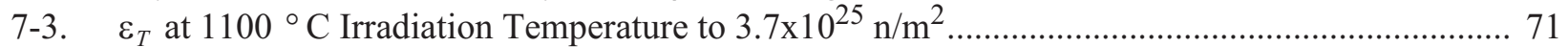

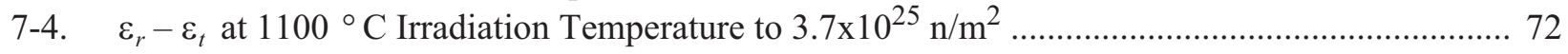

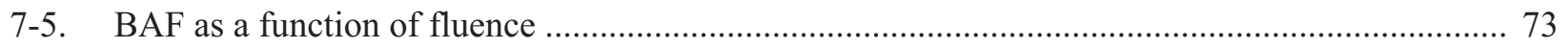

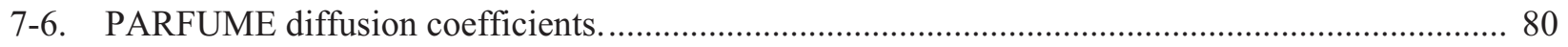




\section{ACRONYMS}

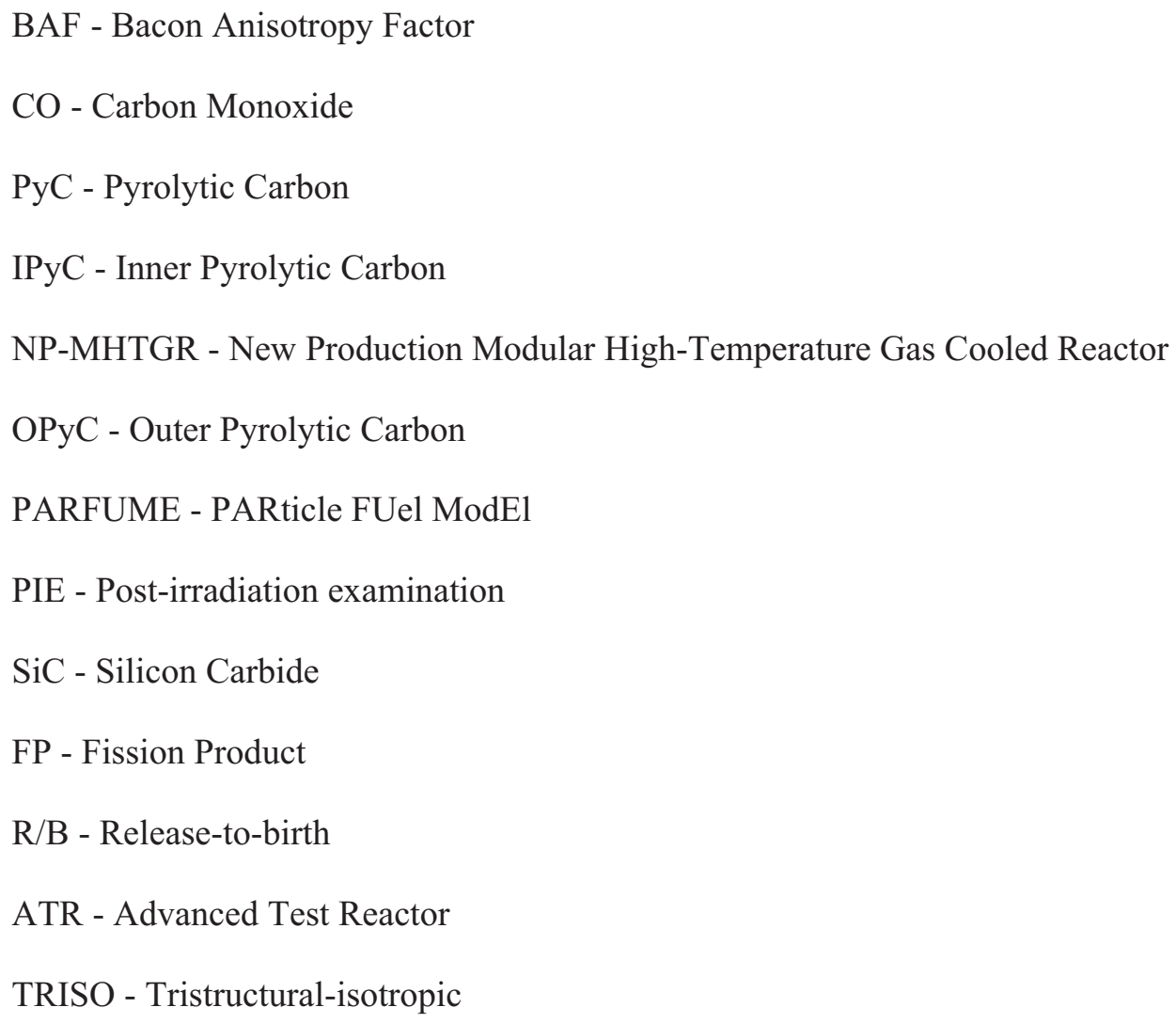




\section{NOMENCLATURE}

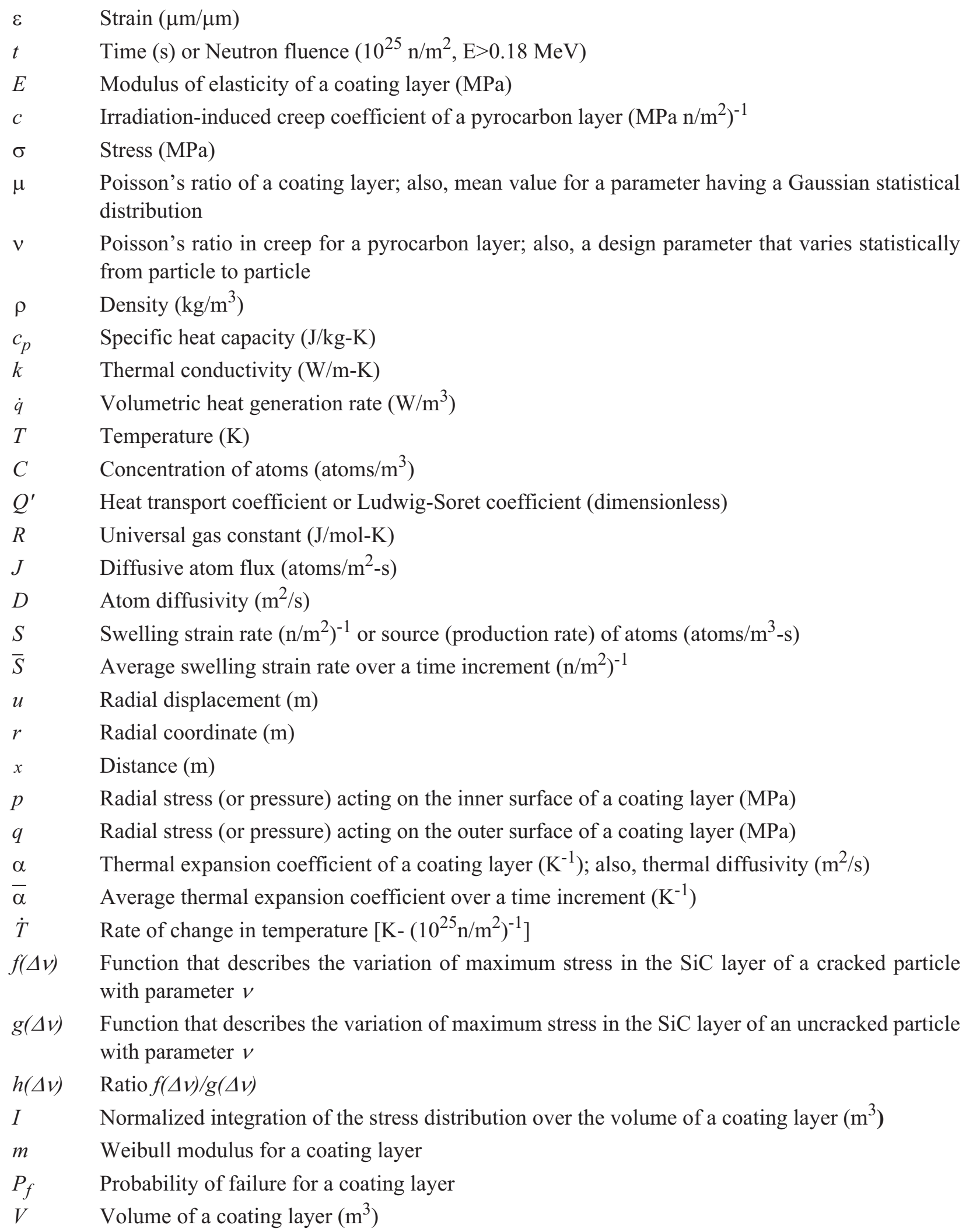


$\sigma \quad$ Stress in a coating layer (MPa)

$\sigma_{c} \quad$ Maximum principal stress in the volume of a coating layer ( $\left.\mathrm{MPa}\right)$

$\sigma_{u} \quad$ Stress in a coating layer for a normal spherical particle (MPa)

$\sigma_{c v} \quad$ Stress in the SiC layer for a multidimensional particle having all parameters set at mean values for a particle batch (MPa)

$\sigma_{u v} \quad$ Stress in the $\mathrm{SiC}$ layer for an intact spherical particle having all parameters set at mean values for a particle batch $(\mathrm{MPa})$

$\sigma_{o} \quad$ Weibull characteristic strength for a coating layer $\left(\mathrm{MPa}-\mathrm{m}^{3 / \mathrm{m}}\right)$

$\sigma_{m s} \quad$ Effective Weibull mean strength for a coating layer (MPa)

$\sigma_{i} \quad \mathrm{i}=1,2,3$, Principal stress components in three orthogonal directions ( $\left.\mathrm{MPa}\right)$

$\Delta v \quad$ Variation in parameter $v$ from its mean value

$\lambda \quad$ Strength for a coating layer in a random particle as sampled from a Gaussian distribution (MPa)

$\lambda_{s} \quad$ Mean strength for a coating layer having a Gaussian strength distribution (MPa)

$\theta \quad$ Latitude angle (radians)

$\phi \quad$ Azimuth angle (radians)

Subscripts

$r \quad$ radial

$t \quad$ tangential

I IPyC layer

$S \quad$ SiC layer

O OPyC layer

$a \quad$ inner surface of a coating layer

$b \quad$ outer surface of a coating layer

B buffer

k kernel 


\section{INTRODUCTION}

PARFUME, the "PARticle FUel ModEl," is being developed as an advanced gas-cooled reactor fuel performance modeling and analysis code. This report presents the theory used to develop the code, including theoretical descriptions of numerical and analytical models.

The coating layers of a fuel particle, which surround the fuel kernel and buffer, consist of an inner pyrolytic carbon (IPyC) layer, a silicon carbide (SiC) layer, and an outer pyrolytic carbon (OPyC) layer. These layers act as a pressure vessel for fission product gases as well as a barrier to the migration of other fission products. The quality of the fuel can be characterized by how well the number of failures of the particles during reactor operation is minimized. Therefore, the PARFUME code has been developed to determine the failure probability of a population of fuel particles, accounting for all viable mechanisms that can lead to particle failure. The code accounts for these calculated particle failures in determining the diffusion of fission products from the fuel through the particle coating layers, and through the fuel matrix to the coolant boundary.

Coated particle fuel exhibits statistical variations in physical dimensions and material properties from particle to particle due to the nature of its fabrication process. Its behavior is also inherently multidimensional, further complicating development of the model. The objective in developing PARFUME is to physically describe both the mechanical and physico-chemical behavior of the fuel particle under irradiation, while capturing the statistical nature of the fuel. Several mechanisms have been identified that can potentially lead to particle failure, including cracking of the IPyC during irradiation, debonding of the IPyC from the $\mathrm{SiC}$ layer during irradiation, buildup of internal fission gas pressure, kernel/SiC interaction resulting from the amoeba effect, and thinning of the $\mathrm{SiC}$ layer due to fission product/SiC interactions. This report describes the theory behind the current capabilities of the code that are used ultimately to determine fuel particle performance.

This document represents the initial version of the PARFUME Theory and Model Basis Report. More detailed discussions will be provided in future revisions.

\subsection{Code Description}

\subsubsection{Basic Fuel Particle Behavior}

A typical TRISO-coated particle is shown in Figure 1-1. Several physical phenomena influence the behavior of the particles, including fission gas production and irradiation effects. For example, fission gas pressure builds up in the kernel and buffer regions, while the IPyC, SiC, and OPyC act as structural layers to retain this pressure. The basic behavior modeled in PARFUME is shown schematically in Figure 1-2. The IPyC and OPyC layers both shrink and creep due to irradiation of the particle, while the $\mathrm{SiC}$ response is essentially limited to elastic behavior. The pressure generally increases as irradiation of the particle progresses, thereby contributing to a tensile hoop stress in the $\mathrm{SiC}$ layer. Countering the effect of the pressure load is the shrinkage of the IPyC during irradiation, which pulls inward on the SiC. Likewise, shrinkage of the OPyC causes it to push inward on the SiC. Failure of the particle is expected to occur if the stress in the $\mathrm{SiC}$ layer reaches the fracture strength of the $\mathrm{SiC}$. Failure of the $\mathrm{SiC}$ results in an instantaneous release of elastic energy that should be sufficient to cause simultaneous failure of the pyrocarbon layers. These effects are described using material, thermal, and physico-chemical models. 


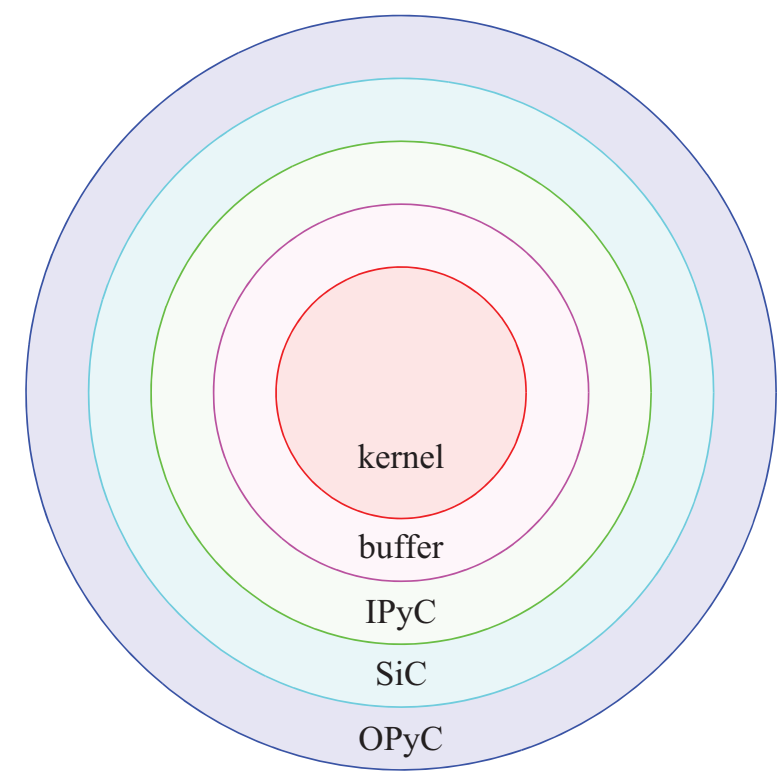

Figure 1-1. Typical TRISO-coated fuel particle geometry.

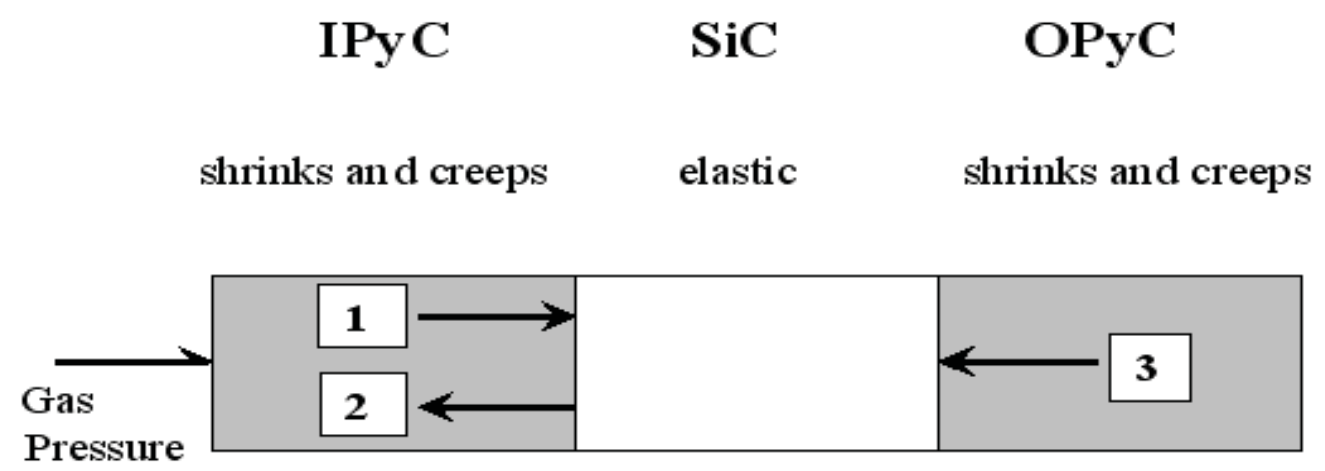

1 Gas pressure is transmitted through the IPyC

2 IPyC shrinks, pulling a way from the $\mathrm{SiC}$

3 OPyC shrinks, pushing in on $\mathrm{SiC}$

Figure 1-2. Behavior of coating layers in fuel particles. 


\subsubsection{Basic Fuel Element Behavior}

The fuel particles, described in Section 1.1.1, are embedded in a spherical, cylindrical, or plate-type graphite matrix; collectively, the fuel particles and matrix are referred to as a fuel element. A fuel element is subject to a temperature distribution that results from the heat generated in the fuel particles and conditions at the element boundaries. A typical prismatic core fuel element is shown in Figure 1-3. The prismatic core consists of a graphite moderator with coolant channels and fuel compacts containing thousands of randomly distributed fuel particles.

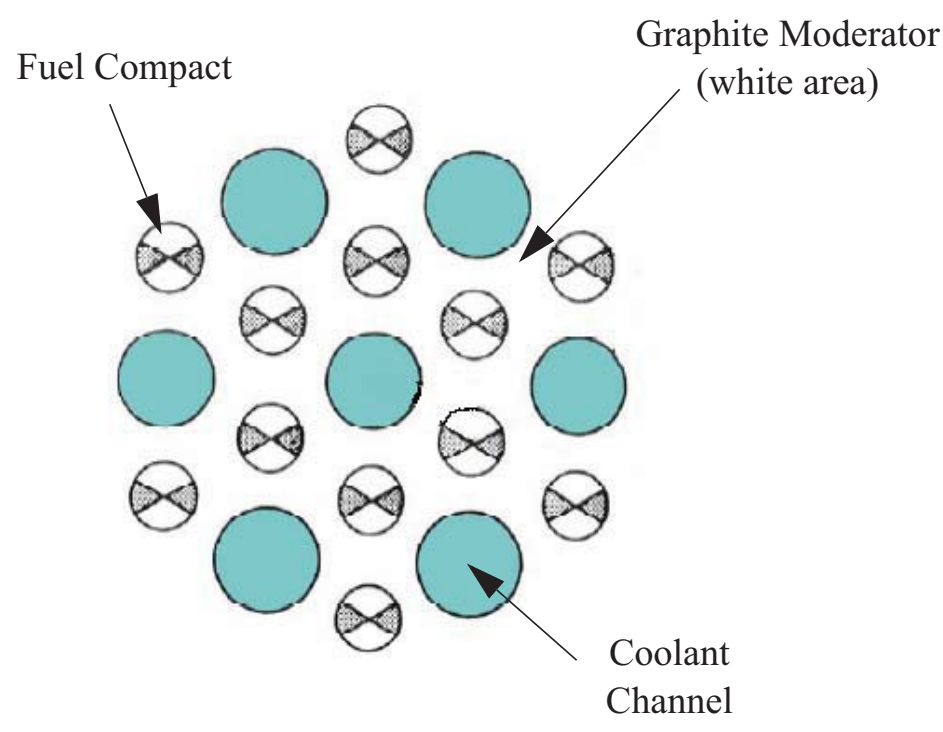

Figure 1-3. Typical element geometry (Prismatic Core).

\subsubsection{General Solution Procedure}

The general solution procedure used by PARFUME consists of the basic processes depicted in the flow chart of Figure 1-4. A general description of each process is given below, while detailed descriptions are provided in the Sections identified in Figure 1-4.

\subsubsection{Compute Fuel Element "Macro" Temperature Profile}

Once the fuel element geometry and temperature boundary conditions are identified, the general heat conduction equation, represented by Equation (1-1), is used to compute the macro temperature distribution throughout the fuel element. The volumetric heat generation rate consists of the total heat generation rate of all the particles.

$\rho c_{p} \frac{\partial T}{\partial t}=k \bullet \nabla^{2} T+\dot{q}$ 


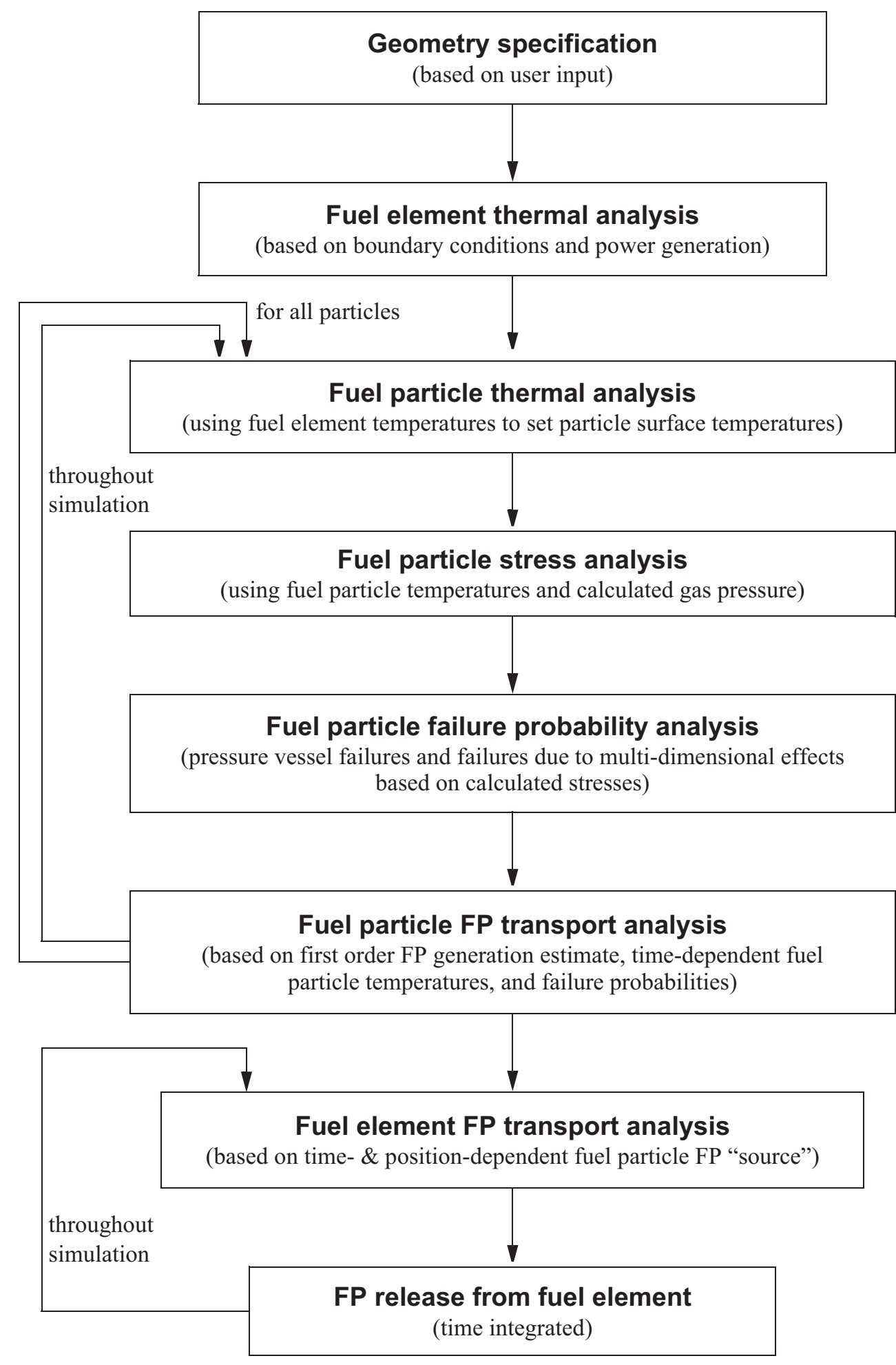

Section 2

Section 3

Section 4

Section 5

\section{Section 6}

Figure 1-4. PARFUME calculational flow chart. 


\subsubsection{Compute Fuel Particle "Micro" Temperature Profile}

Once the macro temperatures for the fuel element are determined, the general heat conduction equation is used to compute the micro temperature profile (using a quasi-steady state assumption). Specifically, the term on the left-hand side of Equation (1-1) (time rate of change of temperature) is assumed to be zero, resulting in Poisson's equation. Therefore, two boundary conditions are required, i.e. the surface temperature of the particle surface and the spatial temperature gradient at the geometric line of symmetry. The particle surface temperature is obtained from the "macro" temperature distribution computed from Equation (1-1), while the spatial temperature gradient at the line of symmetry is set equal to zero. The micro temperature profile accounts for all deformations in the kernel, buffer, and coating layers of the particle as well as the potential for development of a gap between the buffer and the IPyC.

\subsubsection{Compute Particle Stress Distribution}

Once a particle temperature profile is determined, the particle stress distribution is calculated to evaluate whether or not the particle fails. Currently, stress distribution calculations are limited to the buffer, IPyC, $\mathrm{SiC}$, and OPyC though the effect of kernel swelling is included. Strain contributions from several sources are included.

The system of equations used to compute the stress distribution in a spherical particle include constitutive relationships (describing elastic, irradiation-induced, and thermal strain), strain-displacement equations, and the equilibrium stress equation. The two component strain equations (i.e., constitutive relationships) take into account elastic, irradiation-induced creep, irradiation-induced swelling, and thermal strain. The radial strain-rate equation, consisting of four strain-rate terms, is shown in Equation (1-2). Note that the pressure contribution to strain as the result of fission gases and $\mathrm{CO}$ is accounted for in the displacement and stress relationships presented in Section 4.

$\frac{\partial \varepsilon_{r}}{\partial t}=\frac{1}{E}\left(\frac{\partial \sigma_{r}}{\partial t}-2 \mu \frac{\partial \sigma_{t}}{\partial t}\right)+c\left(\sigma_{r}-2 v \sigma_{t}\right)+S_{r}+\alpha_{r} \dot{T}$

Table 1-1 summarizes the strain contributions associated with each fuel particle structure. Because creep and swelling in the $\mathrm{SiC}$ layer are small relative to that of $\mathrm{PyC}$ and because of uncertainty in these properties for $\mathrm{SiC}$, the $\mathrm{SiC}$ strains do not currently include contributions from creep or swelling. The fuel particle kernel is not considered to deform structurally, though a model is included in PARFUME to predict kernel volumetric changes over time.

The stress state in a fuel particle is dependent on the internal gas pressure that exists during either normal reactor operation or an accident condition. Gas pressures are calculated according to the RedlichKwong equation of state and account for the generation of $\mathrm{CO}$ and the release of noble fission product gases. 
Table 1-1. Fuel Particle Strain Contributions

\begin{tabular}{|c|c|c|c|c|}
\hline Fuel Particle Component & $\begin{array}{c}\text { Elastic } \\
\text { Strain }\end{array}$ & $\begin{array}{c}\text { Creep } \\
\text { Strain }\end{array}$ & $\begin{array}{c}\text { Swelling } \\
\text { Strain }\end{array}$ & $\begin{array}{c}\text { Thermal } \\
\text { Strain }\end{array}$ \\
\hline kernel & No & No & Yes & No \\
buffer & Yes & Yes & Yes & Yes \\
IPyC & Yes & Yes & Yes & Yes \\
SiC & Yes & No & No & Yes \\
OPyC & Yes & Yes & Yes & Yes \\
\hline
\end{tabular}

\subsubsection{Compute Failure Probability}

Once stresses have been determined, they are used in conjunction with Weibull statistics to determine particle failure probabilities in the fuel performance model. Assuming that the fuel particle failures follow a Weibull statistical distribution, the failure probability (for example of the $\mathrm{SiC}$ layer) is computed by inserting the calculated stress into Equation (1-3), where the characteristic strength $\left(\sigma_{o}\right)$ and Weibull modulus $(m)$ are determined from experimental data.

$P_{f}=1-e^{-\int_{V}\left(\frac{\sigma}{\sigma_{o}}\right)^{m} d V}$

\subsubsection{Compute Fission Product Diffusion}

In the fission product diffusion calculations, PARFUME estimates fission product gas release due to both recoil and diffusion. It also incorporates the release of fission product gases from failed particles and from uranium contamination in the fuel matrix material.

Once the fission product generation is determined, then the fission product transport calculations are performed. The simulation of fission product transport via diffusion from the fuel through the particle coating layers to the surrounding fuel element graphite matrix, and finally to the coolant boundary is accomplished using the following fundamental transport equation of Equation (1-4), where the flux is driven by fission product concentration gradients and temperature gradients as shown in Equation (1-5).

$\frac{\partial C}{\partial t}=-\nabla \bullet J+S$

$J=-D\left(\nabla C+\frac{Q^{\prime} C}{R T^{2}} \nabla T\right)$

Similar to the temperature profile analyses, "micro" and "macro" diffusion analyses are performed. The micro analysis is based on a model having five different materials (kernel, buffer, IPyC, SiC, and 
OPyC). The macro solution is based on a model having two materials, the graphite containing fuel particles and the surrounding graphite without fuel particles.

Results from all of the diffusion analyses are integrated over time to produce a total fission product release from the fuel element. This is done for each of the fission products under consideration.

\subsection{Quality Assurance}

A Software Quality Assurance Plan will be developed and implemented to assure that the final software application will satisfy quality requirements.

\subsection{Document Organization}

Section 2 of this report describes the various reactor geometries that are treated in the code. Section 3 describes the finite difference thermal models that are used to calculate temperature distributions in the fuel matrix and the quasi-steady state models used to calculate temperature distributions through the fuel particles. Section 4 presents the analytical solution that is used to calculate stresses and displacements in the coating layers of a fuel particle and the various failure mechanisms that are considered in the code. Section 5 presents the statistical method that is used to estimate stresses in particles that undergo multidimensional behavior and describes algorithms that are used to evaluate particle failure due to the various failure mechanisms. It also presents methods used to determine particle failure probabilities, which include the Monte Carlo method, a full integration numerical method, and a fast integration method. Finally, it explains how these failure probability determinations are linked to the fission product diffusion calculations. Section 6 describes the fission product diffusion models used to calculate diffusion through particle coating layers and through the fuel matrix. Section 7 presents material properties that are used in the code for the thermal, mechanical, and diffusion models. 


\section{GEOMETRIES}

The basic fuel particle geometry, described in Section 1.1.1, consists of a kernel of $\mathrm{UO}_{2}$ or UCO surrounded by a buffer and three structural layers (IPyC, $\mathrm{SiC}, \mathrm{OPyC})$. The particles are embedded in a spherical, cylindrical, or plate-type graphite matrix. Collectively, the fuel particles and matrix are referred to as a fuel element. Note that the spherical graphite matrix containing fuel particles is called a "pebble;" approximately 15,000 coated particles are contained within one pebble.

Some definitions:

- fuel particle: kernel + buffer $+\mathrm{IPyC}+\mathrm{SiC}+\mathrm{OPyC}$

- fuel matrix: graphite + embedded fuel particles

- fuel element: fuel matrix + surrounding (unfueled) graphite shell

- fuel elements represent the "macro" level

- fuel particles represent the "micro" level

\subsection{Configurations}

Within PARFUME, three different "macro" fuel element geometries (i.e., plane, cylindrical, and spherical) may be simulated in one dimension as indicated in Figure 2-1. Each macroscopic geometry is modeled by averaging spherical fuel particles, void regions (i.e., coolant channels), and the graphite matrix. This modeling technique is necessary in order to capture the physical behavior of the fuel elements while eliminating the complexities (i.e., computational requirements) of modeling the detailed composition of the fuel element.

\subsubsection{Plane Configuration}

A plane geometry option (i.e., PLANEGEOM) is provided to allow simulation of plate-type fuel. It is assumed that the plate will consist of a center fueled region bounded on both sides by a non-fueled region. The centerline of the plate therefore represents a line of symmetry for both heat and mass transfer. Although plate-type fuel has become unpopular over the years, some reactors such as the Advanced Test Reactor (ATR), continue to use plate-type fuel. Therefore, the plane geometry option is included in PARFUME for completeness.

\subsubsection{Cylindrical Configuration}

A cylindrical geometry option is provided to allow simulation of a unit cell from a prismatic reactor assembly. As an approximation, an appropriate fraction of six fuel compacts represent an equivalent fueled region in the form of a ring. The outer edge of that ring is symmetric relative to both heat and mass transfer. An unfueled region representing the matrix graphite communicates with the fueled region on the inside edge and the coolant channel on the outside edge. Figure 2-2 depicts the basic geometry used to develop the cylindrical fuel element geometry and the transformed geometry. 


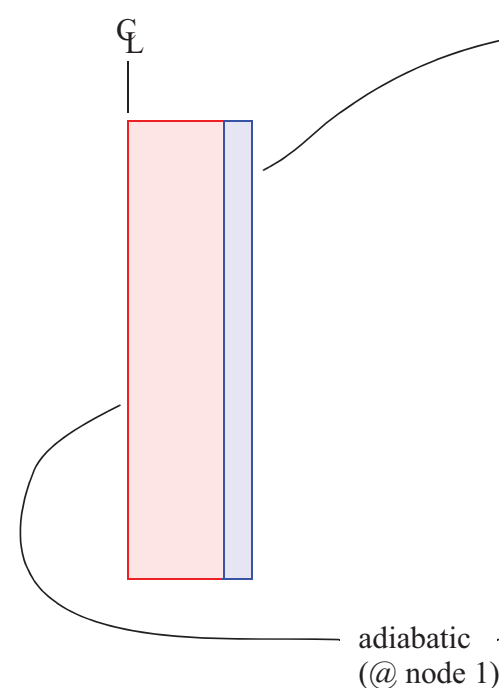

(a) PLANEGEOM

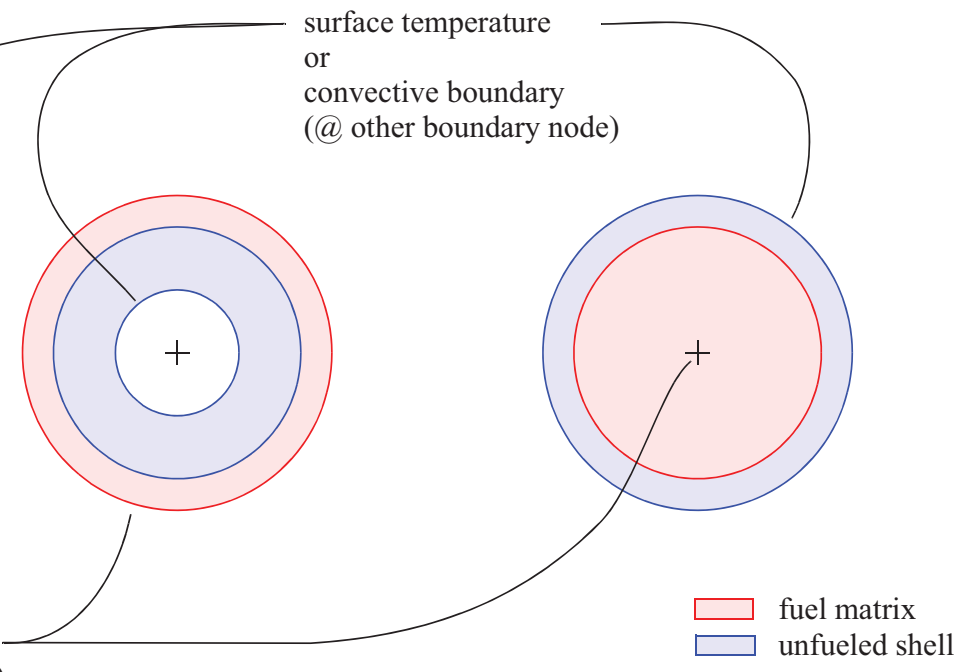

(b) PRISMATIC (c) PEBBLEBED

Figure 2-1. PARFUME "macro" geometries.
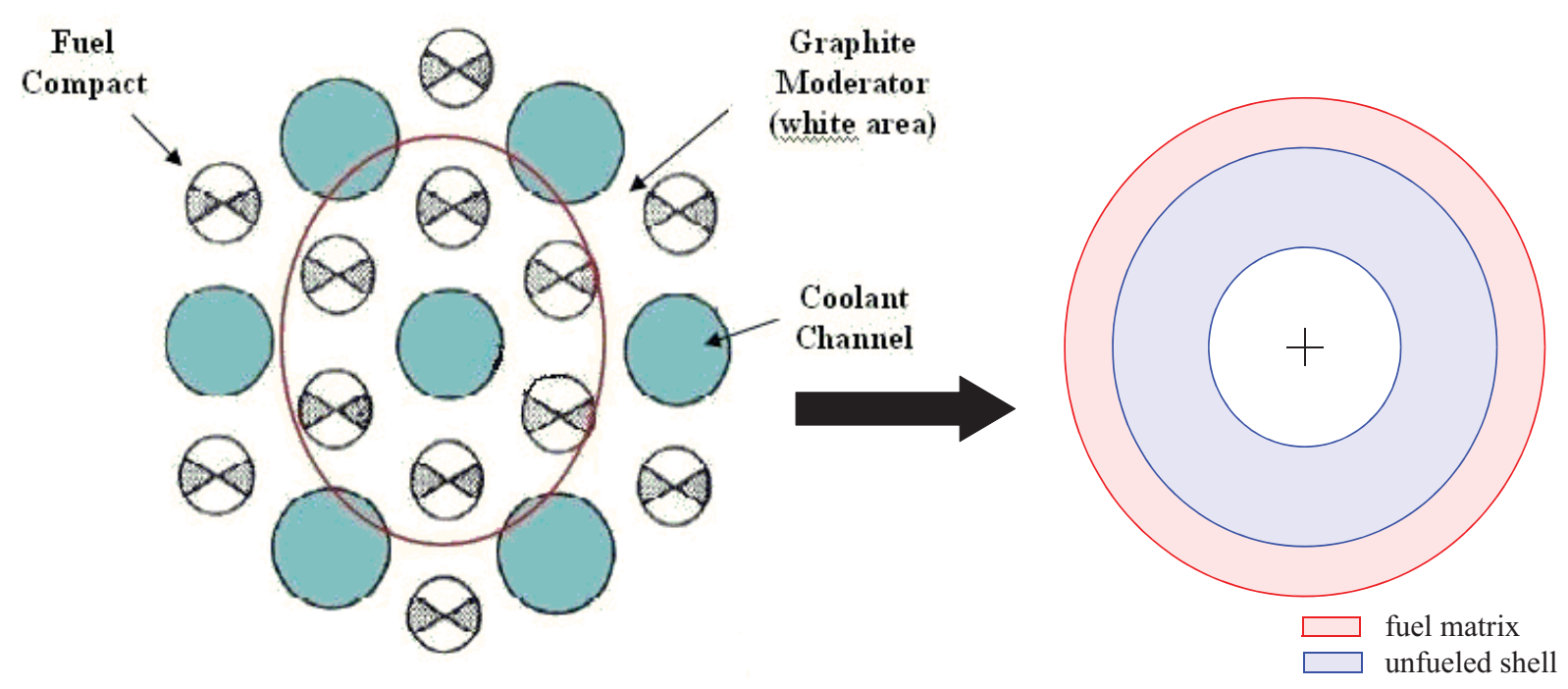

Figure 2-2. PRISMATIC "macro" geometry.

\subsubsection{Spherical Configuration}

A spherical geometry option is provided to allow simulation of a single sphere from a pebble bed reactor. The center of the sphere represents a line of symmetry relative to heat and mass transfer. An unfueled 
region at the outer portion of the sphere communicates with the fueled region on one surface and the coolant on the other surface.

\subsection{Meshing}

The computational mesh represents an important step in the modeling and simulation process; in fact, the accuracy of the results is influenced by the mesh and associated boundary conditions. PARFUME requires generation of two 1-D meshes for heat and mass transfer analyses: the fuel particle "micro" mesh and the fuel element "macro" mesh. An example fuel particle "micro" mesh is presented in Figure 2-3.

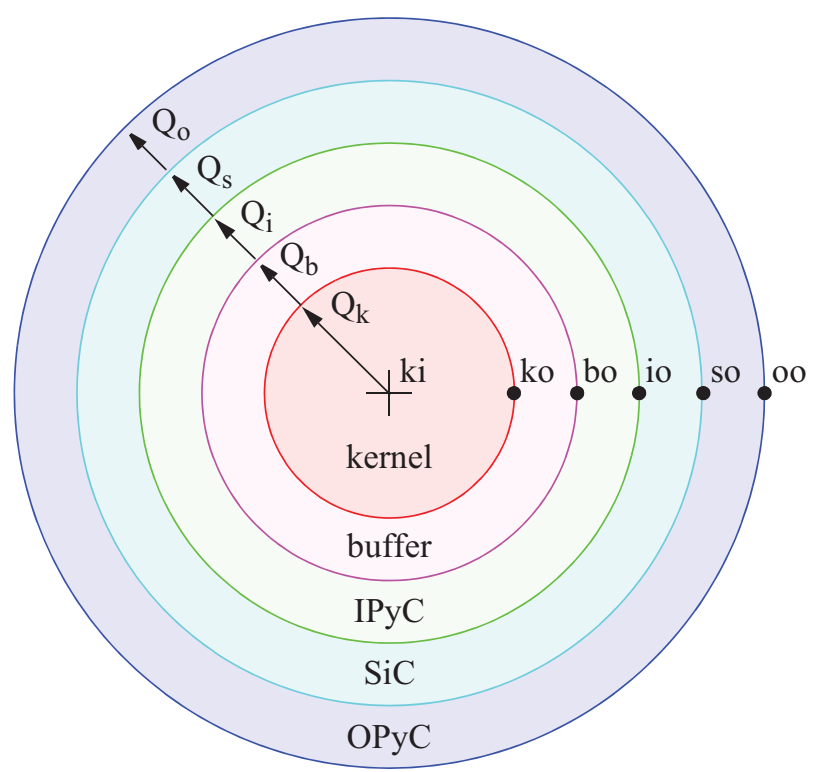

Figure 2-3. Thermal model "micro" mesh

The code currently provides automatic meshing for all analyzed fuel particles. This entails placing nodes on the inner and outer surface of each layer followed by the division of the layer into elements that are nominally $5 \mu \mathrm{m}$ wide. (Obviously, element widths will vary because the material layers will not normally be even increments of $5 \mu \mathrm{m}$.) All interior nodes are then assumed to lie at the midpoint of each element.

The fuel element "macro" mesh provides the user with more flexibility with respect to specifying the number of nodes. Referring to Figure 2-4, a line of symmetry relative to both heat and mass transfer exists in each geometry. Node 1 is always placed on that line of symmetry. Each geometry is then divided into intervals consistent with the number of nodes specified by the user. The last node always aligns with a boundary that, for thermal calculations, may have either a surface temperature specification or a convective surface specification. In diffusion calculations, this boundary has a surface concentration specification. 


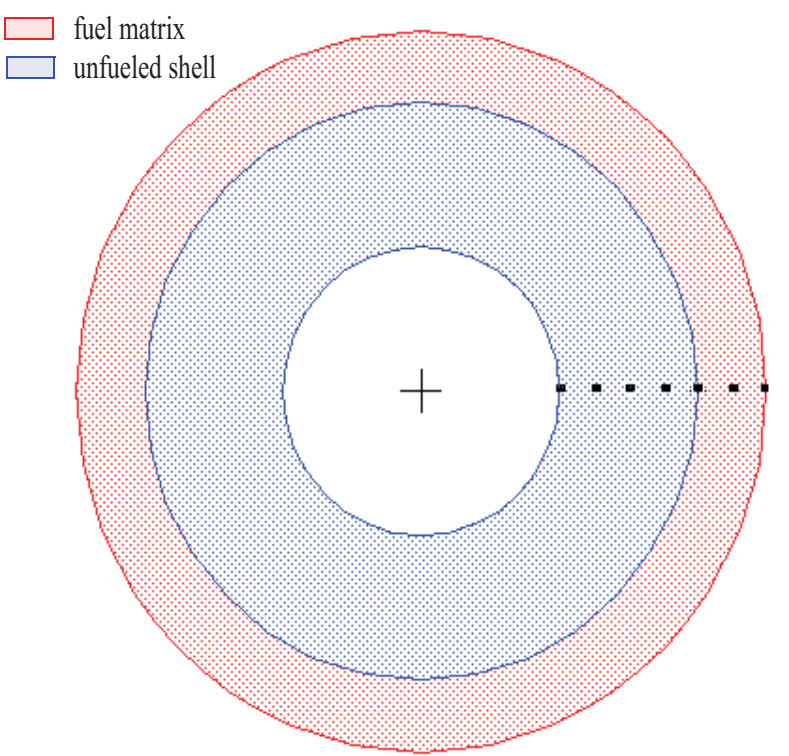

Figure 2-4. PRISMATIC "macro" mesh. 


\section{THERMAL MODELS}

The PARFUME thermal model for the fuel elements is based on a finite difference heat conduction approach with internal heat generation capabilities. It is used to calculate temperatures that are required for determining thermal response of individual fuel particles. Fuel particle results feed a number of temperature-dependent models including calculations for internal pressures, fission product release, and stresses in the coating layers. Temperatures from the fuel element model are also used to evaluate migration of the kernel toward the $\mathrm{SiC}$ layer in the direction of the global temperature gradient (the amoeba effect).

Thermal modeling in PARFUME begins with calculation of a temperature profile through a pebble bed sphere, a prismatic block, or a planar geometry, depending on the reactor type specified by the user. The temperature profile is based on a number of other user inputs including the fuel element geometry, the number of fuel particles within the element, burnup specifications for the irradiation of interest, and the fuel element boundary temperature (i.e., the surface temperature for a pebble bed sphere or the coolant channel surface temperature for a prismatic block). Time-dependent burnup and/or boundary temperature specifications are allowed. The boundary temperature can be provided by the user or can be read from results of a reactor system analysis, for example a neutronic or thermal-hydraulic analysis, or experimental data. The fuel element profiles account for material property dependence on temperature and/or fluence as appropriate.

The resulting time-dependent fuel element temperature profiles, or the 'macro' gradients, are then used to calculate time-dependent fuel particle temperature profiles, or 'micro' gradients, for each particle analyzed. Each micro gradient is based on a particle surface temperature, which is derived from the macro gradients consistent with the statistically determined particle positions. Because fuel particles are very small, the micro gradients are calculated using a quasi-steady state approach (i.e., time rate of temperature change within the particle is approximated as zero). However, material property dependencies on temperature, pressure, and/or fluence are treated as appropriate.

The capability to predict the potential development of a gap between the buffer and the IPyC is an important feature of the thermal model because such a gap can significantly affect the micro temperature distribution within the particle (i.e., micro temperature gradients). Accordingly, the thermal model simulates all of the major factors in gap development including the net effects of kernel swelling; shrinkage and creep in the buffer, IPyC, and OPyC layers; and the associated kernel/buffer contact pressure. Furthermore, the model accounts for changes in gap conductivity with changes in particle geometry and gap gas composition, pressure, and temperature.

\subsection{Macro Temperatures}

\subsubsection{Finite Difference Solution}

One-dimensional, second order temperature solutions are currently implemented for plane, cylindrical, and spherical geometries, which enables simulation of plate fuel, prismatic reactors, and pebble bed reactors, respectively. Heat conduction equations for these geometries are given by:

$\rho c_{p} \frac{\partial T}{\partial t}=\frac{\partial}{\partial x}\left(k \frac{\partial T}{\partial x}\right)+\dot{q}$ 
$\rho c_{p} \frac{\partial T}{\partial t}=\frac{1}{r} \frac{\partial}{\partial r}\left(k r \frac{\partial T}{\partial r}\right)+\dot{q}$

and

$\rho c_{p} \frac{\partial T}{\partial t}=\frac{1}{r^{2}} \frac{\partial}{\partial r}\left(k r^{2} \frac{\partial T}{\partial r}\right)+\dot{q}$

Equations (3-1) through (3-3) can be re-written as fully-implicit (backward) difference equations in tridiagonal form for any interior node "i" as shown in Figure 3-1.

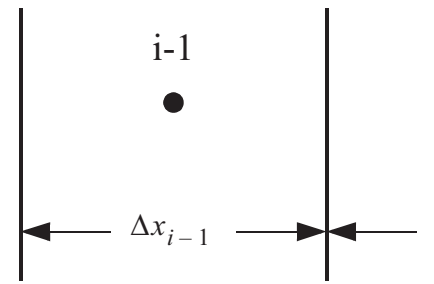

a

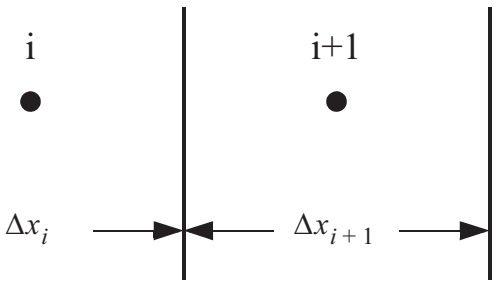

b

Figure 3-1. Finite difference notation used in the macro thermal model.

\subsubsection{For plane geometry.}

Equation (3-1) can be written in difference form as:

$\rho c \frac{\Delta x}{\Delta t} \Delta T=\dot{q} \Delta x+k\left(\frac{\Delta T}{\Delta x}\right)$

Expanding Equation (3-4) for node "i” yields:

$\rho c \frac{\Delta x_{i}}{\Delta t}\left(T_{i}^{n}-T_{i}^{o}\right)=\dot{q}_{i} \Delta x_{i}+\left(\frac{k_{i-1}+k_{i}}{2}\right)\left(\frac{T_{i-1}^{n}-T_{i}^{n}}{\frac{\Delta x_{i-1}}{2}+\frac{\Delta x_{i}}{2}}\right)-\left(\frac{k_{i}+k_{i+1}}{2}\right)\left(\frac{T_{i}^{n}-T_{i+1}^{n}}{\frac{\Delta x_{i}}{2}+\frac{\Delta x_{i+1}}{2}}\right)$

After simplifying and expanding all temperature terms, Equation (3-5) becomes:

$\rho c \frac{\Delta x_{i}}{\Delta t} T_{i}^{n}-\rho c \frac{\Delta x_{i}}{\Delta t} T_{i}^{o}=\dot{q}_{i} \Delta x_{i}+\frac{k_{i-1}+k_{i}}{\Delta x_{i-1}+\Delta x_{i}} T_{i-1}^{n}-\frac{k_{i-1}+k_{i}}{\Delta x_{i-1}+\Delta x_{i}} T_{i}^{n}-\frac{k_{i}+k_{i+1}}{\Delta x_{i}+\Delta x_{i+1}} T_{i}^{n}+\frac{k_{i}+k_{i+1}}{\Delta x_{i}+\Delta x_{i+1}} T_{i+1}^{n}$

Finally, unknown temperatures at the new timestep (denoted by " $n$ ") can be equated to known values. The resulting equation, which is now in tri-diagonal form compatible with PARFUME, is given by:

$-\frac{k_{i-1}+k_{i}}{\Delta x_{i-1}+\Delta x_{i}} T_{i-1}^{n}+\left[\rho_{i} c_{i} \frac{\Delta x_{i}}{\Delta t}+\left(\frac{k_{i-1}+k_{i}}{\Delta x_{i-1}+\Delta x_{i}}\right)+\left(\frac{k_{i}+k_{i+1}}{\Delta x_{i}+\Delta x_{i+1}}\right)\right] T_{i}^{n}-\frac{k_{i}+k_{i+1}}{\Delta x_{i}+\Delta x_{i+1}} T_{i+1}^{n}=q_{i}^{\prime} \Delta x_{i}+\rho_{i} c_{i} \frac{\Delta x_{i}}{\Delta t} T_{i}^{o}$ 


\subsubsection{For cylindrical geometry.}

Equations (3-4) through (3-7) can be re-cast in cylindrical geometry as:

$$
\begin{aligned}
& \rho c \frac{r \Delta r}{\Delta t} \Delta T=\dot{q} r \Delta r+k\left(\frac{r \Delta T}{\Delta r}\right) \\
& \rho c \frac{r_{i} \Delta r_{i}}{\Delta t}\left(T_{i}^{n}-T_{i}^{o}\right)=\dot{q}_{i} r_{i} \Delta r_{i}+\left(\frac{k_{i-1}+k_{i}}{2}\right)\left(\frac{r_{i-1}+r_{i}}{2}\right)\left(\frac{T_{i-1}^{n}-T_{i}^{n}}{\left.\Delta r_{i-1}+\frac{\Delta r_{i}}{2}\right)}-\left(\frac{k_{i}+k_{i+1}}{2}\right)\left(\frac{r_{i}+r_{i+1}}{2}\right)\left(\frac{T_{i}^{n}-T_{i+1}^{n}}{\Delta r_{i}} \frac{\Delta r_{i+1}}{2}\right)\right. \\
& \rho c \frac{r_{i} \Delta r_{i}}{\Delta t} T_{i}^{n}-\rho c \frac{r_{i} \Delta r_{i}}{\Delta t} T_{i}^{o}=\dot{q}_{i} r_{i} \Delta r_{i}+\frac{\left(k_{i-1}+k_{i}\right)\left(r_{i-1}+r_{i}\right)}{2\left(\Delta r_{i-1}+\Delta r_{i}\right)} T_{i-1}^{n}-\frac{\left(k_{i-1}+k_{i}\right)\left(r_{i-1}+r_{i}\right)}{2\left(\Delta r_{i-1}+\Delta r_{i}\right)} T_{i}^{n} \\
& -\frac{\left(k_{i}+k_{i+1}\right)\left(r_{i}+r_{i+1}\right)}{2\left(\Delta r_{i}+\Delta r_{i+1}\right)} T_{i}^{n}+\frac{\left(k_{i}+k_{i+1}\right)\left(r_{i}+r_{i+1}\right)}{2\left(\Delta r_{i}+\Delta r_{i+1}\right)} T_{i+1}^{n} \\
& -\frac{\left(k_{i-1}+k_{i}\right)\left(r_{i-1}+r_{i}\right)}{2\left(\Delta r_{i-1}+\Delta r_{i}\right)} T_{i-1}^{n}+\left[\rho c \frac{r_{i} \Delta r_{i}}{\Delta t}+\frac{\left(k_{i-1}+k_{i}\right)\left(r_{i-1}+r_{i}\right)}{2\left(\Delta r_{i-1}+\Delta r_{i}\right)}+\frac{\left(k_{i}+k_{i+1}\right)\left(r_{i}+r_{i+1}\right)}{2\left(\Delta r_{i}+\Delta r_{i+1}\right)}\right] T_{i}^{n} \\
& -\frac{\left(k_{i}+k_{i+1}\right)\left(r_{i}+r_{i+1}\right)}{2\left(\Delta r_{i}+\Delta r_{i+1}\right)} T_{i+1}^{n}=\dot{q}_{i} r_{i} \Delta r_{i}+\rho c \frac{r_{i} \Delta r_{i}}{\Delta t} T_{i}^{o}
\end{aligned}
$$

\subsubsection{For spherical geometry.}

Equations (3-4) through (3-7) can be re-cast in spherical geometry as:

$$
\begin{aligned}
& \rho c \frac{r^{2} \Delta r}{\Delta t} \Delta T=\dot{q} r^{2} \Delta r+k\left(\frac{r^{2} \Delta T}{\Delta r}\right) \\
& \rho c \frac{r_{i}^{2} \Delta r_{i}}{\Delta t}\left(T_{i}^{n}-T_{i}^{o}\right)=\dot{q}_{i} r_{i}^{2} \Delta r_{i}+\left(\frac{k_{i-1}+k_{i}}{2}\right)\left(\frac{r_{i-1}^{2}+r_{i}^{2}}{2}\right)\left(\frac{T_{i-1}^{n}-T_{i}^{n}}{\Delta r_{i-1}+\frac{\Delta r_{i}}{2}}\right)-\left(\frac{k_{i}+k_{i+1}}{2}\right)\left(\frac{r_{i}^{2}+r_{i+1}^{2}}{2}\right)\left(\frac{T_{i}^{n}-T_{i+1}^{n}}{\Delta r_{i}} \frac{\Delta r_{i+1}}{2}\right) \\
& \rho c \frac{r_{i}^{2} \Delta r_{i}}{\Delta t} T_{i}^{n}-\rho c \frac{r_{i}^{2} \Delta r_{i}}{\Delta t} T_{i}^{o}=\dot{q}_{i} r_{i}^{2} \Delta r_{i}+\frac{\left(k_{i-1}+k_{i}\right)\left(r_{i-1}^{2}+r_{i}^{2}\right)}{2\left(\Delta r_{i-1}+\Delta r_{i}\right)} T_{i-1}^{n}-\frac{\left(k_{i-1}+k_{i}\right)\left(r_{i-1}^{2}+r_{i}^{2}\right)}{2\left(\Delta r_{i-1}+\Delta r_{i}\right)} T_{i}^{n} \\
& -\frac{\left(k_{i}+k_{i+1}\right)\left(r_{i}^{2}+r_{i+1}^{2}\right)}{2\left(\Delta r_{i}+\Delta r_{i+1}\right)} T_{i}^{n}+\frac{\left(k_{i}+k_{i+1}\right)\left(r_{i}^{2}+r_{i+1}^{2}\right)}{2\left(\Delta r_{i}+\Delta r_{i+1}\right)} T_{i+1}^{n} \\
& -\frac{\left(k_{i-1}+k_{i}\right)\left(r_{i-1}^{2}+r_{i}^{2}\right)}{2\left(\Delta r_{i-1}+\Delta r_{i}\right)} T_{i-1}^{n}+\left[\rho c \frac{r_{i}^{2} \Delta r_{i}}{\Delta t}+\frac{\left(k_{i-1}+k_{i}\right)\left(r_{i-1}^{2}+r_{i}^{2}\right)}{2\left(\Delta r_{i-1}+\Delta r_{i}\right)}+\frac{\left(k_{i}+k_{i+1}\right)\left(r_{i}^{2}+r_{i+1}^{2}\right)}{2\left(\Delta r_{i}+\Delta r_{i+1}\right)}\right] T_{i}^{n} \\
& -\frac{\left(k_{i}+k_{i+1}\right)\left(r_{i}^{2}+r_{i+1}^{2}\right)}{2\left(\Delta r_{i}+\Delta r_{i+1}\right)} T_{i+1}^{n}=\dot{q}_{i} r_{i}^{2} \Delta r_{i}+\rho c \frac{r_{i}^{2} \Delta r_{i}}{\Delta t} T_{i}^{o}
\end{aligned}
$$


Based on the notation shown in Figure 3-2, Equation (3-15) and Fourier's law (for spherical coordinates) can be used to determine the temperature distribution within the fuel matrix, given the input for the temperature of the exterior surface of the fuel element.

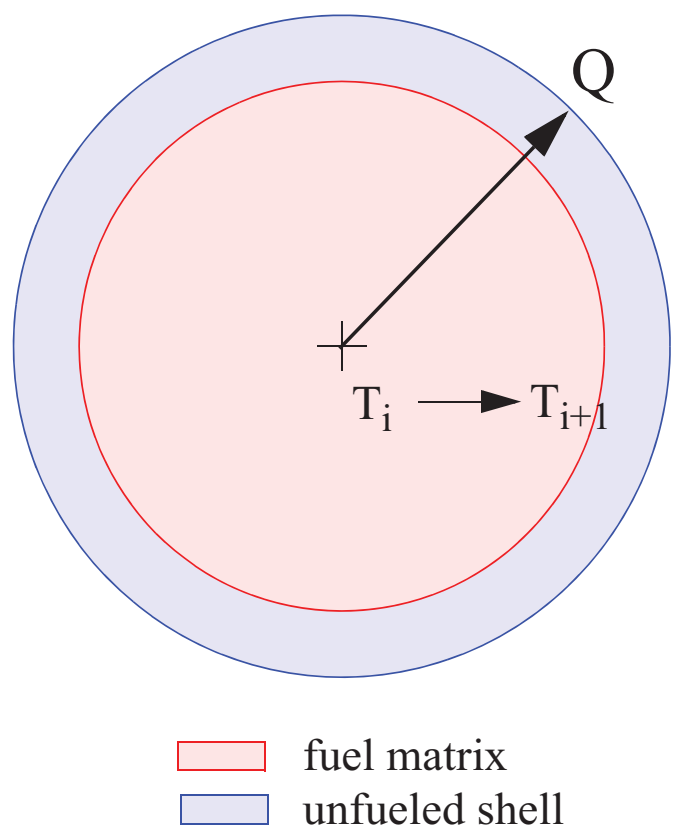

Figure 3-2. Pebblebed Matrix used in the "macro" thermal model

\subsubsection{Macro Temperature Initialization}

The fuel matrix temperature can be initialized by specifying a constant temperature or a temperature distribution. A constant temperature would be specified if, for example, there is interest in analyzing particles undergoing post irradiation heating tests in a furnace operating at a constant temperature. The temperature distribution approach would be used if the user desires to manually input various temperatures at discrete locations within the matrix or if a temperature data profile is available from either experimental data or a systems level code calculation. Note that a file extension ".abq" is required by PARFUME if experimental or "systems level" temperature data are provided.

\subsection{Micro Temperatures}

Temperatures within the TRISO-coated fuel particle are calculated based on a time-dependent exterior surface (OPyC) temperature and a quasi steady-state approach. The exterior surface temperature is derived from the macro temperature solution for the particle position of interest. The quasi steady-state approach 
was adopted as an explicit first-order approximation to minimize calculation time. At this point, a more refined transient temperature solution does not appear to be needed. The temperature solution at the particle centerline and at the interface of each particle layer was developed assuming:

- $\quad$ spherical symmetry (i.e., no particle defects or failures),

- particle materials are isotropic,

- particle material thermal properties are dependent on temperature only,

- the gap between the buffer and the IPyC, if it develops, can be treated as a conducting medium like all other material layers,

- the contact resistance between particle layers is negligible, and

- internal (volumetric) heat generation, if any, exists only in the kernel layer.

The general conduction equation in spherical coordinates with an internal heat source as applicable to this model is given by:

$\frac{\partial T}{\partial t}=\alpha\left\{\frac{1}{r^{2}} \frac{\partial}{\partial r}\left(r^{2} \frac{\partial T}{\partial r}\right)+\frac{1}{r^{2} \sin \theta} \frac{\partial}{\partial \theta}\left(\sin \theta \frac{\partial T}{\partial \theta}\right)+\frac{1}{r^{2} \sin ^{2} \theta} \frac{\partial^{2} T}{\partial \phi^{2}}\right\}+\frac{\dot{q}}{\rho c_{p}}$

The assumptions of spherical symmetry and quasi steady-state allow simplification of Equation (3-16) to read:

$0=\left\{\frac{1}{r^{2}} \frac{d}{d r}\left(r^{2} \frac{d T}{d r}\right)\right\}+\frac{\dot{q}}{k}$

The solution of Equation (3-17) is given by Carslaw and Jaeger ${ }^{1}$ as:

$T=\frac{\dot{q}}{6 k}\left(R^{2}-r^{2}\right)+T_{R}$

where

$R \quad=$ outer radius of the sphere $(\mathrm{m})$ and

$T_{R} \quad=$ temperature $(\mathrm{K})$ at radius $\mathrm{R}$.

Equation (3-18) can be applied to calculate any temperature (T) at any radius in the kernel. The calculation of temperatures on the outer surface of the kernel, and all other radii beyond the kernel, rely on the quasi steady-state assumption that implies:

$\dot{q}_{k} V_{k}=Q_{k}=Q_{b}=Q_{\text {gap }}=Q_{i}=Q_{s}=Q_{o}$

where

$\dot{q}_{k}=$ internal (volumetric) heat generation in the kernel layer $\left(\mathrm{W} / \mathrm{m}^{3}\right)$,

$V_{k}=$ kernel volume $\left(\mathrm{m}^{3}\right)$,

$Q_{k}=$ heat flow through the kernel layer (W),

$Q_{b}=$ heat flow through the buffer layer (W), 
$Q_{\text {gap }}=$ heat flow (W) through the buffer/IPyC gap (if it develops),

$Q_{i} \quad=$ heat flow through the IPyC layer (W),

$Q_{s} \quad=$ heat flow through the $\mathrm{SiC}$ layer $(\mathrm{W})$, and

$Q_{o} \quad=$ heat flow through the OPyC layer (W).

Based on the notation shown in Figure 3-3, Equation (3-19) and Fourier's law (for spherical coordinates) can be used to determine the temperature at the $\mathrm{SiC} /$ OPyC interface, given the input for the temperature of the exterior surface of the TRISO-coated fuel particle. Specifically,

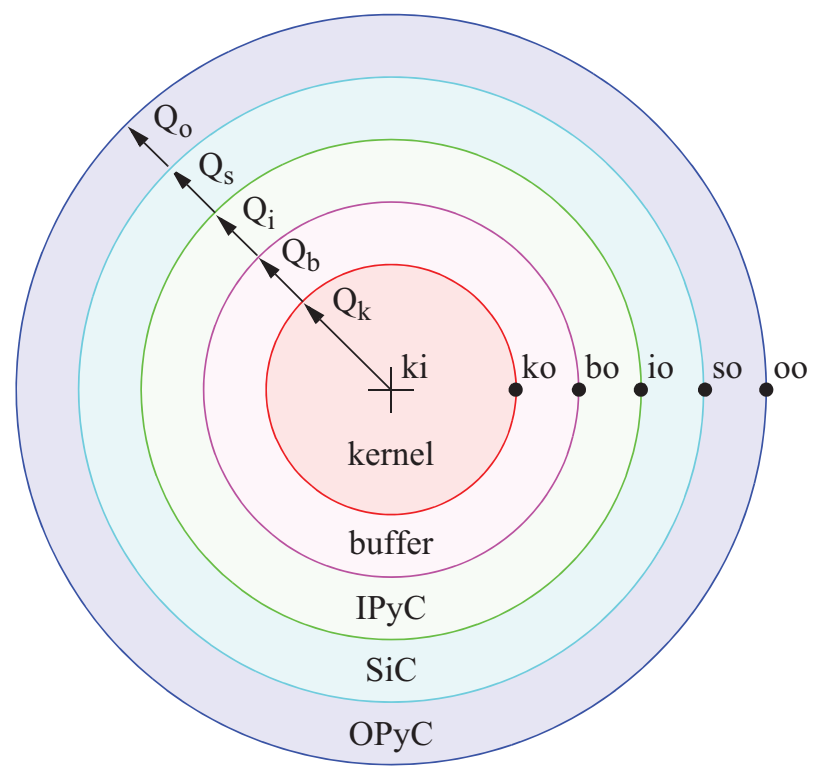

Figure 3-3. Fuel particle notation used in the "micro" thermal model.

$Q=Q_{k}$

$T_{i-1}=T_{i}+\frac{\dot{q}_{k} V_{k}\left(r_{i}-r_{i-1}\right)}{4 \pi r_{i-1} r_{i} k}$

Note that in instances where computational nodes are placed at the interface of each fuel particle layer (i.e., Figure 3-3), with no interior layer nodes, Equation (3-21) reduces to:

$T_{s o}=T_{o o}+\frac{\dot{q}_{k} V_{k}\left(r_{o o}-r_{s o}\right)}{4 \pi r_{s o} r_{o o} k_{o}}$

where

$r_{\text {so }}=$ radius at the outer edge of the $\mathrm{SiC}$ layer,

$r_{o o}=$ radius at the outer edge of the OPyC layer,

$k_{o}=$ conductivity of the OPyC layer, 
$T_{\text {so }} \quad=$ temperature at the outer edge of the $\mathrm{SiC}$ layer, and

$T_{o o}=$ temperature at the outer edge of the OPyC layer.

Equation (3-21) permits the direct (explicit) calculation for the temperature at the outer edge of the SiC layer. Equations similar to those given in (3-20) and (3-21) can be solved sequentially to allow explicit calculation of temperatures at the outer edge of the IPyC layer $\left(T_{i o}\right)$, at the outer edge of the buffer/IPyC gap, if one develops, at the outer edge of the buffer layer $\left(T_{b o}\right)$, and at the outer edge of the kernel layer $\left(T_{k o}\right)$. Given those results, the temperature at the kernel centerline $\left(T_{k i}\right)$ can be calculated with use of Equation (3-18) given by:

$$
T_{k i}=\frac{\dot{q}_{k}}{6 k_{k}} r_{k o}^{2}+T_{k o}
$$

where

$k_{k}=$ conductivity of the kernel layer and

$r_{k o}=$ radius at the outer edge of the kernel layer.

This approach is used to calculate fuel particle temperatures of interest using "old time" values for conductivity. The resulting temperatures are then used to update conductivities and refined temperatures are calculated. This process is repeated until temperatures converge.

It should be noted that the thermal conductivity of the buffer-to-IPyC gap, if one develops, is calculated based on gap temperature, pressure, and gas composition. Temperature, pressure, and gas composition are calculated in the code as functions of time. Correlations used to calculate the gap conductivity were based on coding extracted from the MATPRO Library. ${ }^{2}$

An example of results from the thermal model are shown in Figure 3-4, where kernel centerline temperatures were calculated as functions of particle power and burnup. In this case, fuel particles with a diameter of $780 \mu \mathrm{m}$ were assumed to contain UCO kernels with a diameter of $350 \mu \mathrm{m}$. A volume average irradiation temperature of $1250{ }^{\circ} \mathrm{C}$ was also assumed. This effectively sets the temperature on the outer surface of each particle. The increase in kernel centerline temperatures as shown was due primarily to increases in the size of the gap between the buffer and the IPyC as a result of buffer shrinkage with increasing fast fluence and power being generated in the particle. At high particle powers that might be expected in very accelerated irradiations, the model predicts that the kernel centerline temperature can be as much as $200{ }^{\circ} \mathrm{C}$ higher even though the average temperature is $1250^{\circ} \mathrm{C}$. 


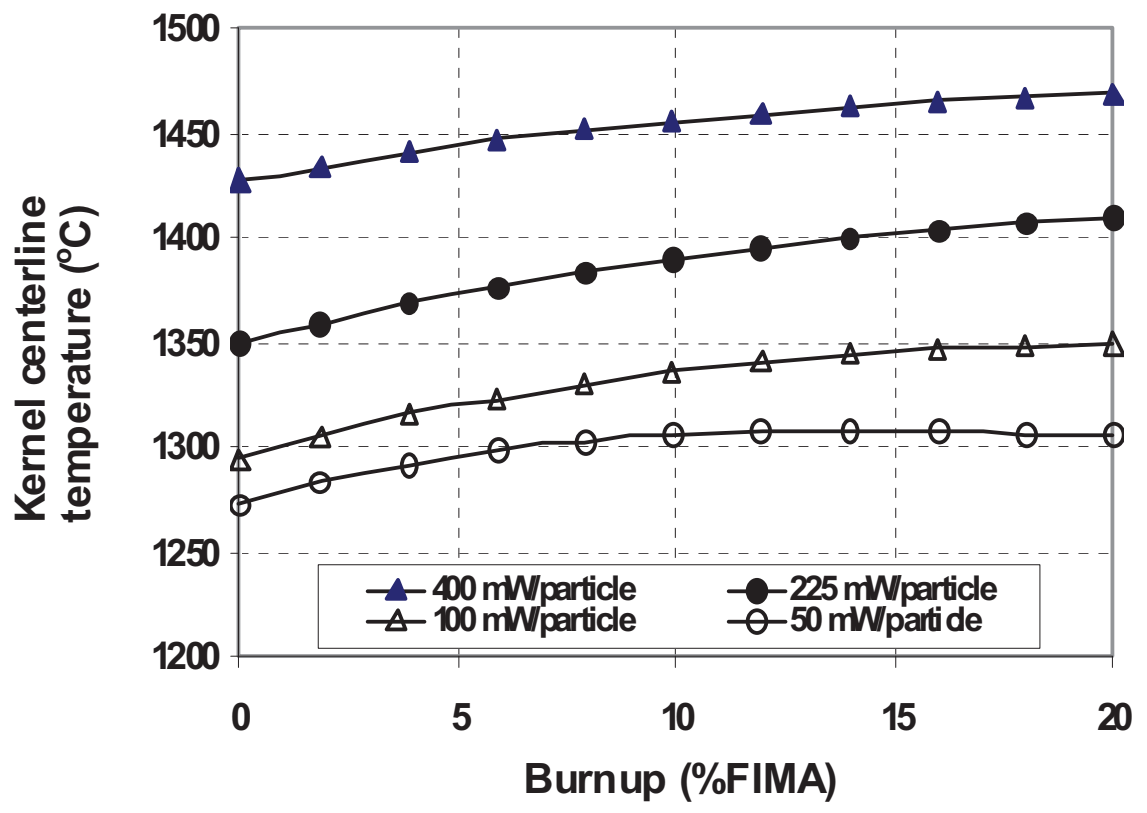

Figure 3-4. Kernel centerline temperatures as functions of particle power and burnup. 


\section{STRUCTURAL MODELS}

A key element of the PARFUME program is a closed form solution that calculates stresses and displacements in the coating layers of a one-dimensional (symmetrical) spherical particle. ${ }^{3,4}$ This solution accounts for the irradiation-induced creep and swelling in the pyrocarbon layers in addition to the elastic behavior of the three layers of a TRISO-coated particle. To treat situations where the particle temperature varies throughout irradiation, the solution allows for time-varying temperatures and material properties and includes the effect of differential expansion among the layers. ${ }^{4}$

\subsection{Background}

Early models of coated fuel particles used iterative numerical procedures to include the effects of pyrocarbon creep and swelling in determining stresses in the coating layers. ${ }^{5,6}$ Iterative procedures, though, are cumbersome to apply when treating statistical variations in Monte Carlo sampling of large particle populations. Bongartz simplified the stress analysis with a closed-form solution based on the assumption of a rigid SiC layer, which enhanced the speed of Monte Carlo calculations ${ }^{7}$. Miller and Bennett subsequently derived a closed-form solution for a three-layer particle that allows for elastic deformation in the $\mathrm{SiC}$ layer $^{3}$ and is well suited for Monte Carlo simulations.

The solution of Miller and Bennett includes stresses that result from irradiation-induced creep and swelling of the PyC layers, internal pressure due to fission gas release, external ambient pressure, and elastic behavior of all three coating layers. As originally formulated, it solved for stresses at a point in time in a single step that starts at the beginning of irradiation. Though this makes for a very efficient solution, it is subject to a number of limitations. It does not allow material properties, such as the elastic moduli of the coating layers, to change with time (or fluence). Nor does it address situations where the irradiation temperature changes with time. A changing temperature significantly affects the stress evolution over time, and induces differential thermal expansion stresses in the layers. A further limitation is the simplifying assumption that Poisson's ratio in creep for the pyrocarbons is 0.5 . The stresses and displacements in the coating layers are sensitive to this parameter, and experimental evidence suggests that the actual value could start at 0.5 but decrease suddenly with irradiation. ${ }^{8}$ It is desirable, therefore, to allow this parameter to assume any realistic value that could change with time.

An updated solution ${ }^{4}$, as described below, removes all of the limitations described above. Additionally, the solution is presented in a manner that would enable its application to a particle having any number of coating layers, not just the three layers of a TRISO-coated particle. The basic approach used is to resolve the solution into time increments, using stresses calculated at the end of an increment as initial conditions for the following increment. The solution remains closed-form, and is used in PARFUME to calculate stresses and displacements in TRISO-coated fuel particles. Applying the solution incrementally through irradiation allows the material properties and irradiation temperature to change with time. The solution allows Poisson's ratio in creep for the pyrocarbon layers to be set to any realistic value that can change with time. With these capabilities, it has been demonstrated to perform efficiently in particle failure probability determinations. 


\subsection{Stress Distribution Theory and Derivation}

\subsubsection{Governing equations and solution}

As in Reference 3, stress-strain relationships for the two components (radial and tangential) of strain in the spherical geometry of a TRISO particle, including Poisson effects, are as follows (see Nomenclature for definitions):

$\frac{\partial \varepsilon_{r}}{\partial t}=\frac{1}{E}\left(\frac{\partial \sigma_{r}}{\partial t}-2 \mu \frac{\partial \sigma_{t}}{\partial t}\right)+c\left(\sigma_{r}-2 v \sigma_{t}\right)+S_{r}+\alpha_{r} \dot{T}$

$\frac{\partial \varepsilon_{t}}{\partial t}=\frac{1}{E}\left((1-\mu) \frac{\partial \sigma_{t}}{\partial t}-\mu \frac{\partial \sigma_{r}}{\partial t}\right)+c\left[(1-v) \sigma_{t}-v \sigma_{r}\right]+S_{t}+\alpha_{t} \dot{T}$

The four terms on the right-hand side of Equations (4-1) and Equation (4-2) represent the strains modeled in PARFUME for the pyrocarbon layers:

- The first term represents elastic strain caused by radial and tangential stress components.

- The second term represents irradiation-induced creep strain resulting from the stress components.

- The third term represents irradiation-induced swelling strain.

- The fourth term represents strain caused by thermal expansion.

The strains due to anisotropic thermal expansion accommodate temperature changes that may occur during irradiation. These equations incorporate the secondary creep (creep strain rate is proportional to the stress) that characterizes the pyrocarbon material.

The strain-displacement relationships and equilibrium requirements for a spherical system complete the description of the behavior of the pyrocarbon layers: ${ }^{9}$

$\varepsilon_{r}=\frac{\partial u}{\partial r}$

$\varepsilon_{t}=\frac{u}{r}$

$\frac{\partial \sigma_{r}}{\partial r}+\frac{2}{r}\left(\sigma_{r}-\sigma_{t}\right)=0$ 
The same equations describe behavior of the SiC layer except that creep and swelling terms are generally omitted. Note that if the swelling or thermal expansion in a layer is isotropic, the radial and tangential strain components can be set equal.

The following series solution is initially assumed:

$u(r, t)=\sum_{i=0}^{\infty} u_{i}(r) t^{i}$

$\sigma_{r}(r, t)=\sum_{i=0}^{\infty} \sigma_{r i}(r) t^{i}$

$\sigma_{t}(r, t)=\sum_{i=0}^{\infty} \sigma_{t i}(r) t^{i}$

where $i$ is the term number and is an exponent on time $t$.

The $t^{0}$ term is included in these summations to accommodate the presence of internal or external pressures at time zero. In Reference 3, the simplification of setting Poisson's ratio in creep ( $v$ ) equal to 0.5 was made, which would make the pyrocarbons incompressible as they creep. Material properties used in the PARFUME code for the coating layers are generally obtained from Reference 25, which recommends the use of 0.5 for $v$ but acknowledges that some other sources prescribe a lower value. Calculations have shown that a lower value can significantly lower the stresses in the coating layers.

Therefore, this derivation is modified to allow any value (from 0 to 0.5 ) for this parameter. With the incremental solution derived here, the parameter $v$ can be varied as desired throughout irradiation. Incorporating this generalization into the derivation modifies Equations (11) and (12) of Reference 3 and results in the following:

$\frac{d^{2} u_{i}}{d r^{2}}+\frac{2 d u_{i}}{r d r}-\frac{2}{r^{2}} u_{i}=\frac{2}{E r}(1-2 \mu) f_{i}+\frac{2}{r} c(1-2 v) \frac{f_{i-1}}{i}$

$f_{i+1}=-\frac{c E(1-v)}{(1-\mu)} \frac{f_{i}}{(i+1)}+\frac{E\left[\left(S_{r}\right)_{i}-\left(S_{t}\right)_{i}+\left(\alpha_{r} \dot{T}\right)_{i}-\left(\alpha_{t} \dot{T}\right)_{i}\right]}{(1-\mu)(i+1)}$, where $f_{0}=0$ 
In these expressions, the functions for swelling and thermal expansion strain rates have been expanded into series, such as $S_{r}=\sum\left(S_{r}\right)_{i} t^{i}$. As before, a function $F(t)$ is defined as follows:

$$
F(t)=\sum_{i=1}^{\infty} f_{i} t^{i}
$$

This function includes the necessary physics for determining stresses that are affected by creep, swelling, and thermal expansion deformation of the coating layers.

The displacement equation of Reference 3 becomes:

$u_{i}=A_{i} r+\frac{B_{i}}{r^{2}}+\left[\frac{2(1-2 \mu)}{3 E} f_{i}+\frac{2 c(1-2 v)}{3 i} f_{i-1}\right] r \ln r$

while the following equation remains unchanged:

$\sigma_{t i}(r)-\sigma_{r i}(r)=\frac{3\left(r_{2} r_{3}\right)^{3}}{r^{3}\left(r_{2}{ }^{3}-r_{3}{ }^{3}\right)}\left(p_{i}-q_{i}-\frac{2}{3} f_{i} \ln \frac{r_{2}}{r_{3}}\right)+\frac{f_{i}}{3}$

Following the process of Reference 10, Equations (4-12) and (4-13) can be used to develop a closedform analytical solution, which evolves from simplification of the series solution, and solves for radial displacement at any radial location in a spherical shell that exhibits swelling and creep in addition to normal elastic behavior:

$u(r, t)=K_{1} p+K_{2} q+K_{3} \int p c d t+K_{4} \int q c d t+K_{5} \int\left(S_{r}+\alpha_{r} \dot{T}\right) d t+K_{6} \int\left(S_{t}+\alpha_{t} \dot{T}\right) d t+K_{7} F(t)$

$F(t)=\left[F\left(t^{n-1}\right)-a_{0}\right] e^{-\frac{c E(1-v)}{1-\mu}\left(t-t_{n-1}\right)}+a_{0}$

where the coefficients $K_{i}$, which are dependent on the geometry and properties of the layer and on the radius $r$, are given by Equations A-1 through A-7 of the Appendix, and the analytical formulation for $F(t)$ is presented in Section 4.2.3. It is noted that the coefficient $K_{7}$ vanishes at the layer surfaces. The stresses 
(or pressures) $p$ and $q$ acting at the layer surfaces are treated as positive outward. If the layer does not creep or swell (such as the SiC layer), this equation reduces to that of a pressurized thick elastic shell.

The fact that the series solution of Equations (4-6) through (4-8) evolves into a closed form solution for the displacement in Equation (4-14) and for stresses in Equations (4-31) and (4-32) below suggests that the solution could be developed using a different mathematical approach. It may be feasible, for example, that with appropriate manipulations the solution could be developed using the LaPlace transform method. The key though is to produce Equations (4-14), (4-31), and (4-32) by whichever means, since these give the general solution at any time for the displacement and stresses in a single layer. From these, the solution for any multi-layered particle can be derived. It is noted that the contribution to displacement from the integral terms of Equation (4-14) can grow steadily with time as energy is imparted to the layer. In a single layer, these displacements could grow without bound until failure is reached. Deformations in the threelayer coating system of a TRISO-coated particle, though, are controlled by the restraint of the stiff SiC layer.

\subsubsection{Internal Gas Pressure}

It is evident from Equation (4-14) that the displacements, and therefore stresses, in a fuel particle are dependent on the internal gas pressure.

Particle internal gas pressures are calculated according to the Redlich-Kwong equation of state. ${ }^{11}$ Parameters utilized by this equation of state are derived from the critical temperature and pressure of each gas specie ${ }^{12}$ occupying void volume within the particle. PARFUME considers the generation of CO and the release of the noble fission product gases, xenon and krypton, in this pressure calculation.

PARFUME calculates fission product gas release due to both recoil and diffusion. Direct fission recoil from the kernel to the buffer is accounted for by geometrical considerations and fission fragment ranges derived from compiled experimental data ${ }^{13}$. Diffusive release is calculated according to the Booth equivalent sphere diffusion model ${ }^{14}$ which utilizes an effective diffusion coefficient formulated by Turnbull. ${ }^{15}$ This effective diffusion coefficient accounts for intrinsic, thermal and radiation-enhanced diffusion.

One of three algorithms may be chosen for calculating CO production: the General Atomics (GA) model, Proksch model, or HSC model. The GA model is a simple temperature dependent correlation ${ }^{16}$ that is used primarily for comparison to historic evaluations. The Proksch model is also used primarily for comparison to historic German evaluations. ${ }^{17}$ The final model, referred to as the "HSC model" in this report, is a detailed model derived from thermochemical free energy minimization calculations performed by the HSC code. ${ }^{18}$ This CO production model considers burnup, temperature, uranium enrichment, and fuel composition in the calculation. Input to the HSC code consisted of elemental fission product inventories generated by the MOCUP ${ }^{19}$ code which couples the MCNP ${ }^{20}$ and ORIGEN2 ${ }^{21}$ codes.

\subsubsection{Function $F(t)$}

The function $F(t)$ contains the physics required to appropriately capture effects of irradiation-induced creep and swelling deformation. There is a function $F(t)$ for each of the pyrocarbon layers. If the SiC layer is treated as an isotropic elastic medium, then its $F(t)$ becomes zero. 
Substituting Equation (4-10) into (4-11) and differentiating with respect to $t$ gives the following equation for the function $F(t)$ :

$\frac{d F}{d t}+\frac{c E(1-v)}{1-\mu} F=\frac{E}{1-\mu}\left(\overline{S_{r}}+\overline{\alpha_{r} \dot{T}}-\overline{S_{t}}-\overline{\alpha_{t} \dot{T}}\right)$

The overlines in Equation (4-16) serve to indicate that swelling and thermal expansion strain rates are numerically averaged over the time increment, and are treated as constants through the increment. The general solution to this differential equation is the closed-form function

$F(t)=\left[F\left(t^{n-1}\right)-a_{0}\right] e^{-\frac{c E(1-v)}{1-\mu}\left(t-t_{n-1}\right)}+a_{0}$

where $a_{0}$ for time increment $n$ is:

$a_{0}=\frac{\overline{S_{r}}+\overline{\alpha_{r} \dot{T}}-\overline{S_{t}}-\overline{\alpha_{t} \dot{T}}}{c(1-v)}$

\subsection{General Stress Equations}

\subsubsection{Radial Stresses at Layer Interfaces}

The radial stresses at the $\mathrm{IPyC} / \mathrm{SiC}$ and $\mathrm{SiC} / \mathrm{OPyC}$ interfaces must be determined so that general expressions for radial and tangential stresses, Equations (4-31) and (4-32) below, can be developed. The radial stresses at the interfaces are solved by equating displacements at the interfaces and differentiating the resulting equations with respect to $t$. This results in two simultaneous differential equations as follows:

$\frac{d \sigma_{r O}}{d t}-B_{2} \sigma_{r O}-B_{1} \sigma_{r I}=x(t)$

$\frac{d \sigma_{r I}}{d t}-B_{3} \sigma_{r I}-B_{4} \sigma_{r O}=y(t)$

where the quantities $B_{i}$ are determined from Equations A-8 through A-12 of the Appendix. 
With the functions $x(t)$ and $y(t)$ expressed as follows, which is a suitable representation over a time increment:

$x(t)=x_{0}+x_{1} t$

$y(t)=y_{0}+y_{1} t$

the solution to Equations (4-19) and (4-20) becomes:

$\sigma_{r O}=D_{1} e^{m_{1} t}+D_{2} e^{m_{2} t}+v_{0}+v_{1} t$

$\sigma_{r I}=\frac{\left(m_{1}-B_{2}\right)}{B_{1}} D_{1} e^{m_{1} t}+\frac{\left(m_{2}-B_{2}\right)}{B_{1}} D_{2} e^{m_{2} t}+w_{0}+w_{1} t$

where $x_{0}, x_{1}, y_{0}, y_{1}, m_{1}, m_{2}, v_{0}, v_{1}, w_{0}$, and $w_{1}$ are given by Equations A-13 through A-21. These quantities are constant during a time increment, but change from one increment to the next. In this solution, the SiC layer is assumed to not creep or swell.

Equations (4-19) and (4-20) apply to a three-layer system where there are two interface surfaces. This method of solution, though, can be applied as well to a system with any number of coating layers. The result is a set of simultaneous differential equations of the form of Equations (4-19) and (4-20), where there is an equation for each interface surface. These can be solved using matrix analysis, which yields a set of eigenvalues and eigenvectors for the system. The eigenvalues for the two-equation system above are $m_{1}$ and $m_{2}$.

As discussed above, the solution is applied in time increments, which means that coefficients $D_{1}$ and $D_{2}$ for each increment are determined from the initial conditions for that increment. At the start of irradiation $(t=0)$, the initial values for internal pressure $p$ and external pressure $q$ are applied. At time zero, all integral terms in Equations (4-33) through (4-36) vanish, and the equations are readily solved to give the following for the radial interface stresses at the start of irradiation:

$\sigma_{r O}(0)=\frac{a_{1} c_{1} p-d_{2}\left(b_{1}-a_{2}\right) q}{b_{2} c_{1}-\left(c_{2}-d_{1}\right)\left(b_{1}-a_{2}\right)}$

$\sigma_{r I}(0)=\frac{a_{1}\left(c_{2}-d_{1}\right) p-d_{2} b_{2} q}{\left(b_{1}-a_{2}\right)\left(c_{2}-d_{1}\right)-c_{1} b_{2}}$ 
These become the initial conditions for determining the coefficients $D_{1}$ and $D_{2}$ for the first increment. In subsequent time increments, the radial stresses at the end of an increment become the initial conditions for the next increment. Using Equations (4-23) and (4-24), then, the coefficients for the general time increment $n$ are determined to be:

$D_{1}=\frac{\left(m_{2}-B_{2}\right) X-B_{1} Y}{m_{2}-m_{1}} e^{-m_{1} t_{n-1}}$

$D_{2}=\frac{\left(m_{1}-B_{2}\right) X-B_{1} Y}{m_{1}-m_{2}} e^{-m_{2} t_{n-1}}$

where

$X=\sigma_{r O}\left(t_{n-1}\right)-v_{0}-v_{1} t_{n-1}$

$Y=\sigma_{r I}\left(t_{n-1}\right)-w_{0}-w_{1} t_{n-1}$

and $t^{n-1}$ denotes the time $t$ at the end of the previous increment $n-1$. In applying these equations, all material properties, swelling strain rates, thermal expansion strain rates, and known internal and external pressures are numerically averaged over the time increment.

\subsubsection{General Equations for Radial and Tangential Stresses}

Equations (4-23) and (4-24) give the radial contact stresses at the inside and outside surfaces of the SiC layer. Once these equations have been solved, together with $F(t)$ from Equation (4-17), it is possible to determine radial or tangential stresses at any radial location in the coating layers. Note that tangential stresses are needed to determine whether the coating layers fail.

As shown in Reference 10, the following general expressions for radial and tangential stresses in a coating layer can then be developed:

$\sigma_{r}(r, t)=\frac{r_{a}{ }^{3}\left(r_{b}{ }^{3}-r^{3}\right)}{r^{3}\left(r_{b}{ }^{3}-r_{a}{ }^{3}\right)} p-\frac{r_{b}{ }^{3}\left(r_{a}{ }^{3}-r^{3}\right)}{r^{3}\left(r_{b}{ }^{3}-r_{a}{ }^{3}\right)} q-\frac{2}{3}\left[\frac{r_{a}{ }^{3}\left(r_{b}{ }^{3}-r^{3}\right) \ln r_{a}-r_{b}{ }^{3}\left(r_{a}{ }^{3}-r^{3}\right) \ln r_{b}}{r^{3}\left(r_{b}{ }^{3}-r_{a}{ }^{3}\right)}-\ln r\right] F(t)$ 


$$
\begin{aligned}
& \sigma_{t}(r, t)=-\frac{r_{a}^{3}\left(2 r^{3}+r_{b}^{3}\right)}{2 r^{3}\left(r_{b}^{3}-r_{a}^{3}\right)} p+\frac{r_{b}^{3}\left(2 r^{3}+r_{a}^{3}\right)}{2 r^{3}\left(r_{b}^{3}-r_{a}^{3}\right)} q \\
& +\frac{1}{3}\left[\frac{r_{a}{ }^{3}\left(r_{b}^{3}+2 r^{3}\right) \ln r_{a}-r_{b}{ }^{3}\left(r_{a}^{3}+2 r^{3}\right) \ln r_{b}}{r^{3}\left(r_{b}{ }^{3}-r_{a}^{3}\right)}+2 \ln r+1\right] F(t)
\end{aligned}
$$

where $r_{a}$ and $r_{b}$ are the inner and outer radii of the layer, respectively, and $p$ and $q$ are the radial stresses acting on the inner and outer surfaces of the layer, respectively. Unlike the $F(t)$ of Reference 10, that of Equation (4-17) allows Poisson's ratio in creep ( $v$ ) to be set to any value.

\subsection{General Displacement Equations}

Equation (4-14) is the basic equation used in PARFUME to calculate radial displacement. Displacements are calculated at the radial locations $r_{2}, r_{3}, r_{4}$, and $r_{5}$ so that new values can be determined for these radii at the end of each time increment; radial displacement is not calculated at $r_{1}$ since it is assumed that the inner portion of the buffer moves with the kernel. The updated radii are needed in the thermal and fission product transport analyses of the particle, and are be used in the stress solution.

Equation (4-14) is applied below to the four shell surfaces located at the two interfaces $\left(r=r_{3}, r_{4}\right)$ of the TRISO-coated particle. Note that the IPyC and OPyC layers are assumed to exhibit secondary creep and anisotropic swelling, and all three layers are allowed to exhibit anisotropic thermal expansion.

$\underline{\text { IPyC outer surface }}$

$u_{I}=a_{1} p+a_{2} \sigma_{r I}+a_{3} \int p c_{I} d t+a_{4} \int \sigma_{r I} c_{I} d t+a_{5} \int\left(S_{r I}+\alpha_{r I} \dot{T}_{I}\right) d t+a_{6} \int\left(S_{t I}+\alpha_{t I} \dot{T}_{I}\right) d t$

$\underline{\text { SiC inner and outer surfaces }}$

$u_{I}=b_{1} \sigma_{r I}+b_{2} \sigma_{r O}+b_{3} \int \alpha_{r S} \dot{T}_{S} d t+b_{4} \int \alpha_{t S} \dot{T}_{S} d t$

$u_{O}=c_{1} \sigma_{r I}+c_{2} \sigma_{r O}+c_{3} \int \alpha_{r S} \dot{T}_{S} d t+c_{4} \int \alpha_{t S} \dot{T}_{S} d t$ 
$\underline{\text { OPyC inner surface }}$

$u_{O}=d_{1} \sigma_{r O}+d_{2} q+d_{3} \int \sigma_{r O} c_{O} d t+\mathrm{d}_{4} \int q c_{O} d t+\mathrm{d}_{5} \int\left(S_{r O}+\alpha_{r O} \dot{T}_{O}\right) d t+\mathrm{d}_{6} \int\left(S_{t O}+\alpha_{t O} \dot{T}_{O}\right) d t$

where the coefficients $a_{j}, b_{j}, c_{j}$, and $d_{j}$ are determined by substituting the appropriate radii and material properties into the expressions for $K_{i}$.

\subsection{1. $\mathrm{SiC}$ Displacement $\left(\mathbf{r}_{3}\right.$ and $\left.\mathbf{r}_{4}\right)$}

Once the radial stresses $\sigma_{r O}$ and $\sigma_{r I}$ are determined, then the displacements at the inner and outer surfaces of the $\mathrm{SiC}$ are determined from Equations (4-34) and (4-35).

Because of the integrations in these equations, the displacements at $r_{3}$ and $r_{4}$ are most readily determined by differentiating through with respect to $t$, and calculating the displacements incrementally. For example, Equations (4-34) and (4-35) become:

$\Delta u_{3}=b_{1} \Delta \sigma_{r I}+b_{2} \Delta \sigma_{r O}+b_{3} \alpha_{r S} \dot{T}_{S} \Delta t+b_{4} \alpha_{t S} \dot{T}_{S} \Delta t$

$\Delta u_{4}=c_{1} \Delta \sigma_{r I}+c_{2} \Delta \sigma_{r O}+c_{3} \alpha_{r S} \dot{T}_{S} \Delta t+c_{4} \alpha_{t S} \dot{T}_{S} \Delta t$

\subsubsection{IPyC and OPyC Displacement ( $r_{2}$ and $\left.r_{5}\right)$}

The displacements at $r_{2}$ and $r_{5}$ are obtained from Equations (4-33) and (4-36) except that the coefficients are modified appropriately and the equations are applied incrementally. These become

$\Delta u_{2}=a_{1}{ }^{\prime} \Delta p+a_{2}{ }^{\prime} \Delta \sigma_{r I}+\left(a_{3}{ }^{\prime} p+a_{4}{ }^{\prime} \sigma_{r I}\right) c_{I} \Delta t+a_{5}{ }^{\prime}\left(S_{r I}+\alpha_{r I} \dot{T}_{I}\right) \Delta t+a_{6}{ }^{\prime}\left(S_{t I}+\alpha_{t I} \dot{T}_{I}\right) \Delta t$

$\Delta u_{5}=d_{1}{ }^{\prime} \Delta \sigma_{r O}+d_{2}{ }^{\prime} \Delta q+\left(d_{3}{ }^{\prime} \sigma_{r O}+d_{4}{ }^{\prime} q\right) c_{O} \Delta t+d_{5}{ }^{\prime}\left(S_{r O}+\alpha_{r O} \dot{T}_{O}\right) \Delta t+\mathrm{d}_{6}{ }^{\prime}\left(S_{t O}+\alpha_{t O} \dot{T}_{O}\right) \Delta t$

The coefficients $a_{i}{ }^{\prime}$ and $d_{i}{ }^{\prime}$ are calculated from Equations A-1 through A-6 using dimensions appropriate for the IPyC inner surface and OPyC outer surface, respectively. 


\subsubsection{Modeling of the Buffer-IPyC Gap}

An important aspect of the fuel particle behavior is the development of a gap between the buffer and IPyC layers (i.e., at $r_{2}$ ) during irradiation. The gap is formed because the irradiation-induced shrinkage of the porous buffer exceeds that of the IPyC layer. A significant consequence of this gap is that the low thermal conductivity of the gap region (which is occupied by fission products) contributes to an increase in the kernel temperature. The kernel temperature affects fission gas release, which in turn affects the gas pressure acting on the coating layers and fission product transport through the particle layers and fuel matrix. Because the magnitude of the increase in kernel temperature depends on the size of the gap, PARFUME calculates the gap size throughout irradiation. This is done by calculating a displacement for the outer surface of the buffer layer in addition to displacement $u_{2}$ of the IPyC inner surface.

\subsubsection{Buffer Displacement}

In this calculation, the buffer is assumed to shrink and creep due to irradiation and the fuel kernel is assumed to swell throughout irradiation. It is currently assumed in PARFUME for purposes of this calculation that the inner surface of the buffer moves with the kernel, and that the buffer remains intact throughout irradiation. Application of Equation (4-14) to the inner surface of the buffer gives the following:

$p_{B}=\frac{1}{a_{1 B}}\left[u_{k}-a_{3 B} \int p_{B} c_{B} d t-r_{1} \int\left(S_{B}+\alpha_{B} \dot{T}\right) d t\right]$

where $p_{B}$ is the radial contact stress at the interface of the kernel and buffer; $u_{k}$ is the known displacement of the kernel surface; and $c_{B}, S_{B}$, and $\alpha_{B}$ are creep, shrinkage, and expansion properties for the buffer. The coefficients $a_{1 B}$ and $a_{3 B}$ are obtained by substituting appropriate values into Equations A-1 and A-3.

Because of its porosity, the buffer is assumed to experience no deformation due to the gas pressure. Thus, there is no pressure applied to its outer surface. Also, because the buffer is assumed to be isotropic (per

Reference ${ }^{25}$, for low-density pyrocarbon), the radial components of swelling and thermal expansion strain rate $(S, \alpha \dot{T})$ are set equal to the corresponding tangential components.

The pressure $p_{B}$ can be determined from Equation (4-41) by differentiating through with respect to $t$, and solving the resulting differential equation. This gives the following for the pressure at any time during time increment $n$ :

$p_{B}(t)=\left\{p_{B}\left(t_{n-1}\right)-\frac{1}{a_{3 B} c_{B}}\left[\frac{d u_{k}}{d t}-r_{1}\left(S_{B}+\alpha_{B} \dot{T}\right)\right]\right\} e^{-\frac{a_{3 B}}{a_{1 B} c\left(t-t_{n-1}\right)}}+\frac{1}{a_{3 B} c_{B}}\left[\frac{d u_{k}}{d t}-r_{1}\left(S_{B}+\alpha_{B} \dot{T}\right)\right]$

where $t^{n-1}$ is the time at the start of the increment, and the derivative $d u_{k} / d t$ is treated as a constant through the time increment. Using this contact pressure, the displacement at the outer surface of the buffer $\left(u_{2 B}\right)$ is then determined incrementally from 
$\Delta u_{2 B}=b_{1 B} \Delta p_{B}+b_{3 B} p_{B} c_{B} \Delta t+r_{2 B}\left(S_{B}+\alpha_{B} \dot{T}\right) \Delta t$

where $b_{1 B}$ and $b_{3 B}$ are determined from Equations A-1 and A-3 using dimensions at the outer surface of the buffer, and $r_{2 B}$ is the outer radius of the buffer.

\subsubsection{IPyC Displacement}

The displacement of the IPyC is initially formulated by applying Equation (4-14) in time increments as shown below:

$\Delta u=a_{1} \Delta p+a_{2} \Delta p+a_{3} p c \Delta t+a_{4} q c \Delta t+a_{5}\left(S_{r}+\alpha_{r} T\right) \Delta t+a_{6}\left(S_{t}+\alpha_{r} T\right) \Delta t$

The displacement of the IPyC is determined from Equation (4-44) setting $p$ equal to the internal gas pressure and $q$ equal to the calculated radial stress at the interface between the IPyC and SiC layers.

\subsubsection{Example Gap Behavior}

To demonstrate that PARFUME produces accurate results for the buffer/IPyC gap (assuming accuracy of the assumptions), a finite element analysis was performed using the ABAQUS program. ${ }^{22}$ In this analysis, the buffer was assumed to remain intact and sustain a tangential stress. The Poisson's ratio in creep for the PyC and buffer layers was changed from 0.4 to 0.5 , since the algorithm for creep behavior in ABAQUS permits only this value. As shown in Figure 4-1, results show close agreement for the two analyses

\subsection{Two-layer and One-layer Solutions}

Situations arise in fuel particle evaluations that require stress analysis of a two-layer or one-layer shell. For example, a detachment of the IPyC from the $\mathrm{SiC}$ results in both two-layer ( $\mathrm{SiC} / \mathrm{OPyC})$ and one-layer (IPyC) shells. In such cases, an assessment is made as to whether the IPyC shell can sustain the internal pressure on its own. If it fails, then the internal pressure is applied directly to the two-layer (SiC/OPyC) shell.

Stresses and displacements for a two-layer shell are obtained in the same manner as used for the threelayer shell, i.e., by equating displacements at the interface between layers to solve for the radial stress at the interface. This contact stress, together with the known internal and external pressures, is used to determine displacements at the layer surfaces. The tangential stresses in the layers are determined using Equation (4-32) [and Equation (4-17) for $F(t)$ ]. 
Buffer/IPyC Gap

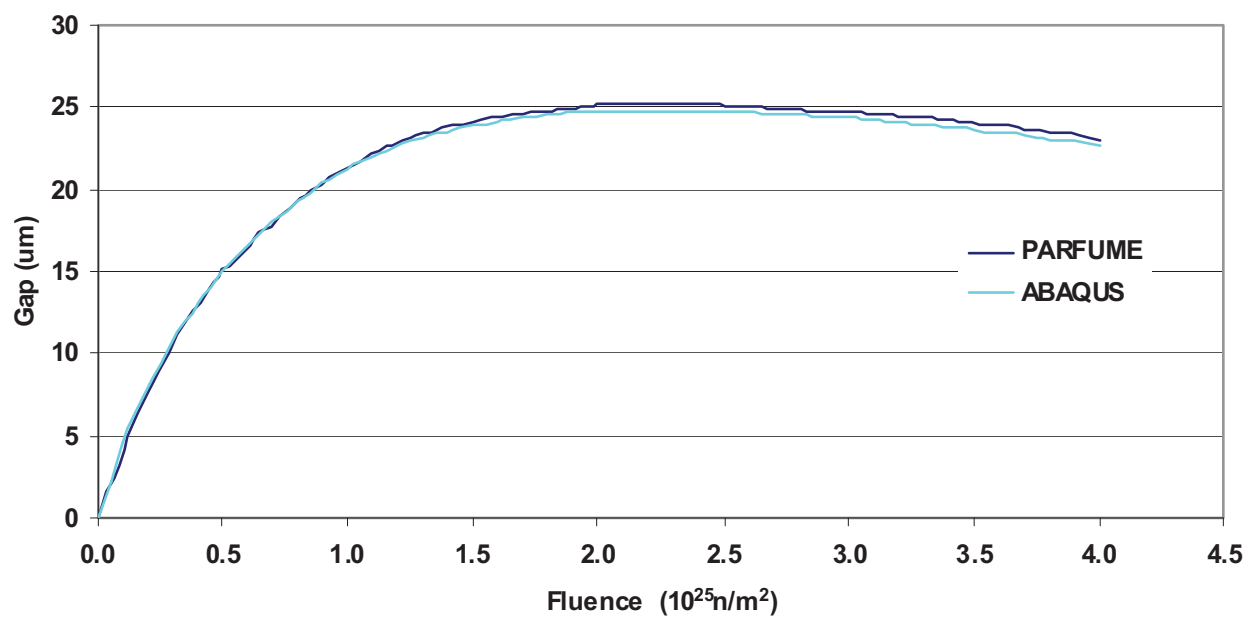

Figure 4-1. PARFUME and ABAQUS comparison.

The radial stress at the $\mathrm{SiC} / \mathrm{OPyC}$ interface for a particle having a debonded $\mathrm{IPyC}$ is as follows:

$\sigma_{r O}=D_{1}{ }^{\prime} e^{m_{1}{ }^{\prime} t}-\frac{x_{0}{ }^{\prime}+x_{1}{ }^{\prime}}{m_{1}{ }^{\prime}}-\frac{x_{1}{ }^{\prime}}{m_{1}{ }^{\prime}} t$

while the radial stress at the $\mathrm{IPyC} / \mathrm{SiC}$ interface for a particle having a debonded $\mathrm{OPyC}$ is:

$\sigma_{r I}=D_{2}{ }^{\prime} e^{m_{2}{ }^{\prime} t}-\frac{y_{0}{ }^{\prime}+y_{1}{ }^{\prime}}{m_{2}{ }^{\prime}}-\frac{y_{1}{ }^{\prime}}{m_{2}{ }^{\prime}} t$

where the primed quantities are determined from Equations A-22 through A-29.

Since there is no radial interface stress to calculate in a one-layer particle, the known internal and external pressures acting on the layer are used directly to determine displacements and tangential stresses.

\subsection{Particle Failure Mechanisms}

The solution described above for stresses in a TRISO-coated particle is one-dimensional, relying on perfect spherical symmetry. Failure of a one-dimensional particle occurs if the internal pressure is high 
enough that the tangential stress in the $\mathrm{SiC}$ layer reaches the $\mathrm{SiC}$ strength for that particle. PARFUME also considers multi-dimensional behavior that has been observed in post-irradiation examination (PIE) of US fuel particles and may contribute to particle failures, such as 1) cracking of the IPyC layer, 2) partial debonding of the IPyC from the SiC layer, 3) an aspherical geometry, and 4) the amoeba effect.

\subsubsection{Pressure Vessel Failure}

A traditional failure mechanism addressed in PARFUME as well as other fuel performance codes is the pressure vessel failure of a one-dimensional spherical particle. The particle is one-dimensional because of perfect symmetry in the tangential and azimuthal directions. Early during irradiation, the shrinkage of the pyrocarbon layers puts the $\mathrm{SiC}$ layer in compression. As irradiation progresses, the irradiation-induced creep of the pyrocarbon layers tends to relieve some of this compressive stress. Additionally, the buildup of fission gas pressure tends to put the coating layers in tension. If the gas pressure increases enough, the tangential stress in the $\mathrm{SiC}$ layer could eventually become tensile. A traditional pressure vessel failure is expected to occur if the tangential stress reaches a value that exceeds the strength of the $\mathrm{SiC}$ for that particle.

\subsubsection{Cracking of the IPyC}

One form of multi-dimensional behavior that is modeled in PARFUME is a radial shrinkage crack in the IPyC. The crack in the IPyC is typical of those observed in post irradiation examination (PIE) of the New Production Modular High-Temperature Gas Cooled Reactor (NP-MHTGR) fuel particles. During irradiation, shrinkage of the initially intact IPyC layer induces a significant tensile stress in that layer. If the stress exceeds the tensile strength of the IPyC layer, then a radial crack develops in the IPyC. The radial crack creates local tensile stresses in the SiC layer that could lead to particle failure. An axisymmetric finite element model used in an ABAQUS ${ }^{22}$ analysis of a cracked three-layer geometry is shown in Figure 4-2. Figure 4-3 plots a time history for the maximum calculated principal stress at a point in the $\mathrm{SiC}$ layer near the tip of the crack. The stress at this point increases as the pyrocarbon layers shrink during irradiation, but eventually peaks as creep in the pyrocarbon layers overcomes the shrinkage effect. The evaluation of particles for potential failure due to a cracked IPyC is discussed in Section 5.3.2.1.

\subsubsection{Partial Debonding of the IPyC from the SiC}

A second form of multi-dimensional behavior modeled in PARFUME is partial debonding between the IPyC and the $\mathrm{SiC}$, which has also been observed in PIE of the NP-MHTGR fuel particles. During irradiation, shrinkage of the IPyC layer induces a radial tensile stress at the interface between the IPyC and $\mathrm{SiC}$ layers. If the stress exceeds the bond strength between layers, then debonding of the IPyC from the $\mathrm{SiC}$ occurs. The debonding process is not likely to be an instantaneous detachment over the entire surface of the interface. Rather, it begins at an initiation point from which the layers progressively unzip during irradiation. An axisymmetric finite element model used in ABAQUS analysis for the debonded geometry is shown in Figure 4-4. The model plotted is a deformed shape as it appears part way through irradiation, after the unzipping process has begun.

In an ABAQUS analysis of a debonded particle, the IPyC and $\mathrm{SiC}$ are initially assumed to be debonded at an arbitrary point in the model. Continued debonding, if it occurs, then progresses from this point. The criterion used is that the next node ahead of the crack tip debonds when the local stress across the interface 


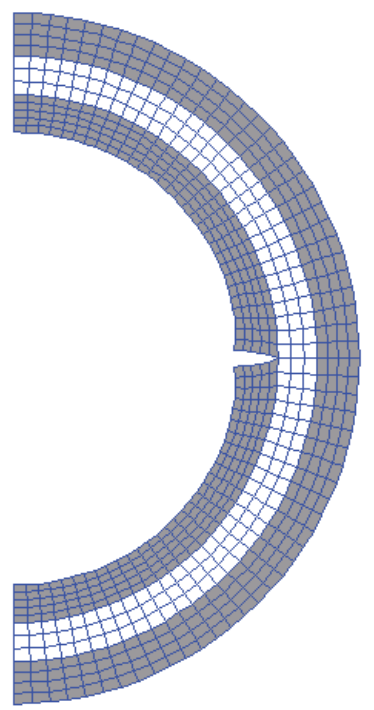

Figure 4-2. Finite element model for fuel particle having radial crack in IPyC layer.

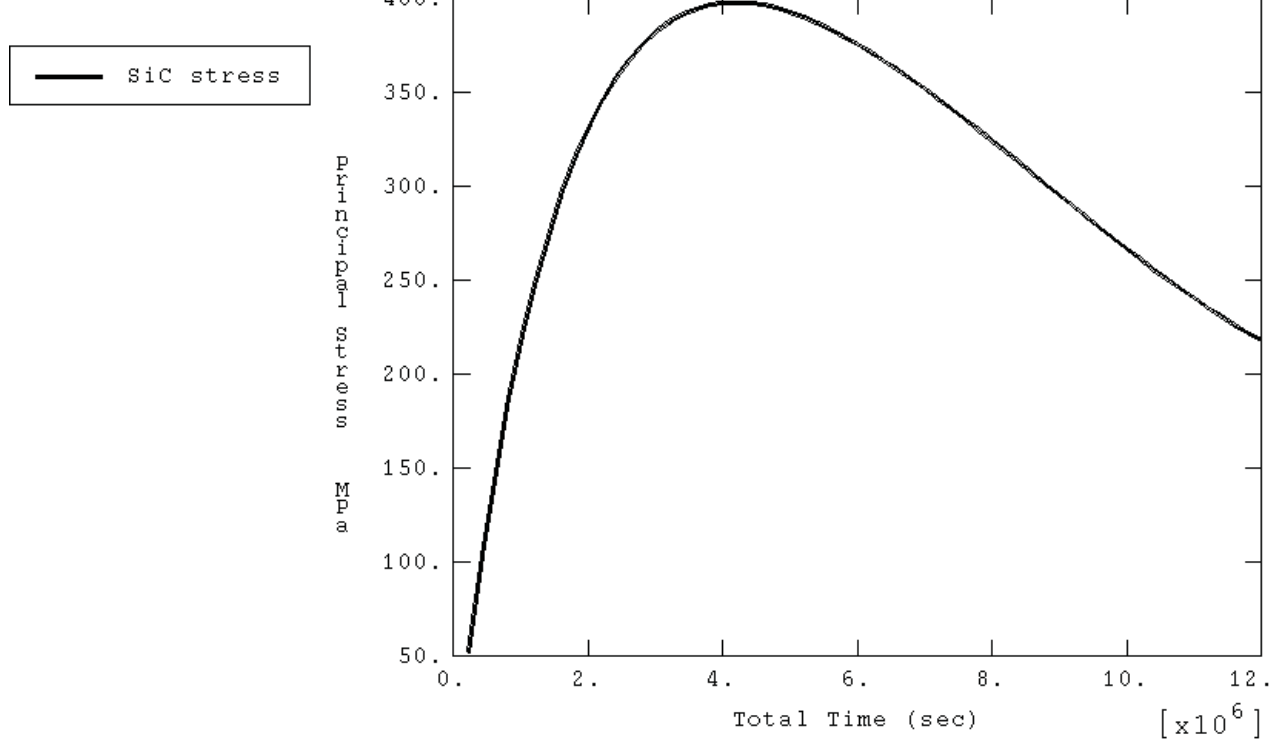

Figure 4-3. Stress history in $\mathrm{SiC}$ layer (near crack tip) for cracked particle.

at a specified distance ahead of the crack tip reaches the specified bond strength. A stress concentration occurs in the $\mathrm{SiC}$ layer at the tip of the debonded region, containing tensile stress components that could contribute to failure of the SiC. Figure 4-5 plots a time history for the maximum calculated principal stress at a point (point 1) on the inner surface of the SiC layer along the debonded path. The stress at this point rose to a peak as the tip of the debonded region passed through this location. With continued unzipping 
between layers, the stress at this location rapidly diminished. Also shown in Figure 4-5 is a stress history at a point further down the debonded path (point 2). As would be expected, the stress at this point peaked at a later time during irradiation. The evaluation of particles for potential failure due to a partially debonded $\mathrm{IPyC}$ is discussed in Section 5.3.2.2.

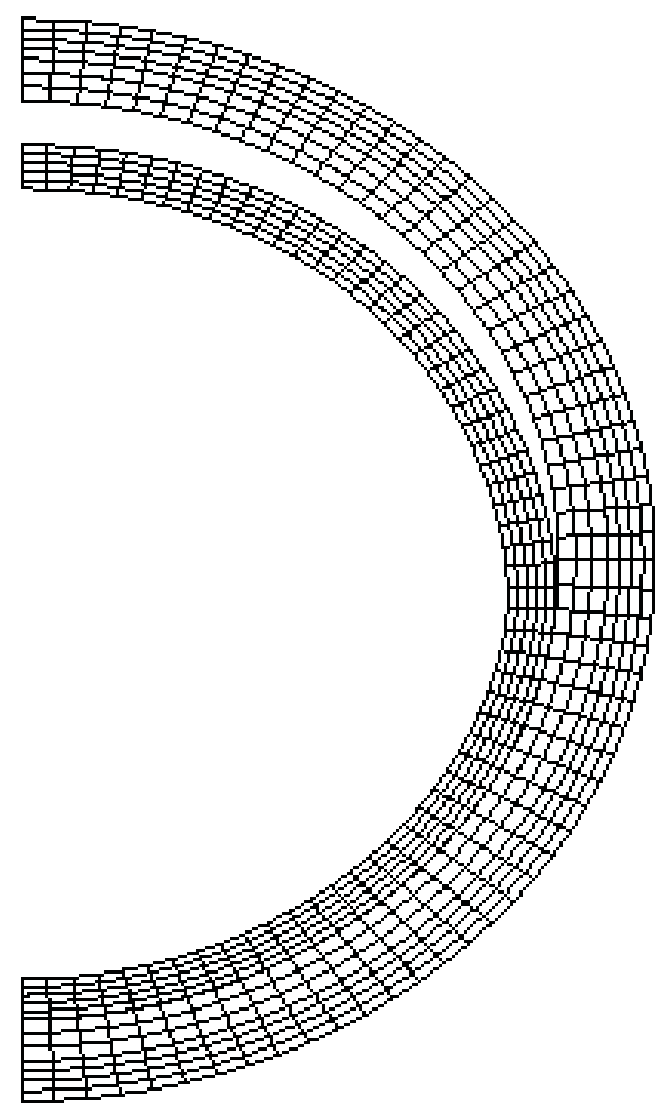

Figure 4-4. Finite element model for a partially debonded particle.

\subsubsection{Pressure Vessel Failure of an Aspherical Particle}

A third form of multi-dimensional behavior modeled in PARFUME is asphericity. The Code incorporates the effects of asphericity for particles that have a flat facet but that are otherwise spherical. Because the effects of an ellipsoidal shape are small in comparison, this type of asphericity is not included in the Code. During irradiation, the faceted portion of the particle acts as a flat plate that retains the internal gas pressure. If the pressure builds up high enough, this results in a local region of tensile stress in the central portion of the plate, which can contribute to particle failures. Unlike failures caused by cracking of the IPyC or partial debonding of the IPyC, which are governed by shrinkage of the pyrocarbons, failures caused by asphericity are controlled by the internal pressure. Therefore, while failures due to IPyC cracking and debonding tend to occur early during irradiation when shrinkage stresses are at their highest, fail- 


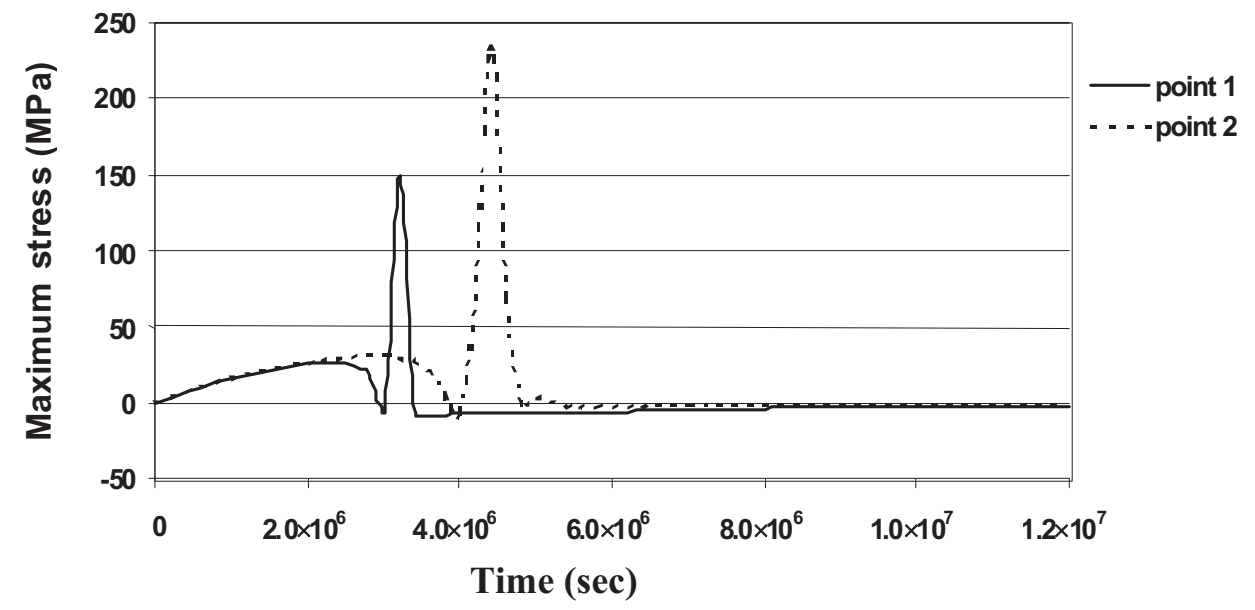

Figure 4-5. Inner $\mathrm{SiC}$ layer stress histories at two points that experience debonding.

ures due to asphericity are likely to occur later when the internal pressure is highest. An axisymmetric finite element model used to analyze for the effects of asphericity is shown in Figure 4-6.

The degree of asphericity for a particle is defined in terms of an aspect ratio, which is the ratio of the major diameter to minor diameter. A reason for defining this parameter is that it is a commonly used measure of the severity of deformity in a particle, and is thereby used as a criterion for particle acceptability. Using dimensions shown in Figure 4-6, the aspect ratio $(A)$ is

$A=\frac{2 R}{R+\sqrt{R^{2}-r^{2}}}$

where $R$ is the outer radius of the particle and $r$ is the radius of the facet. PARFUME allows the user to specify a statistical distribution for the aspect ratio when considering asphericity.

Figure 4-7 plots a time history for the principal stress in the $\mathrm{SiC}$ calculated at the center of the faceted portion of an aspherical (faceted) particle. Also plotted is the time history for the tangential stress at a location removed from the facet. A comparison of these stress histories shows how the facet intensifies the stress in that local region of the particle. Depending on its severity, this stress intensification could contribute to particle failures. The evaluation of particles for potential failure due to asphericity is discussed in Section 5.3.2.3.

\subsubsection{Amoeba Effect}

A final failure mechanism currently considered in the code is failure of the $\mathrm{SiC}$ due to the amoeba effect, which does not involve stress levels in the coating layer. Failure due to the amoeba effect is evaluated by determining the distance that the fuel kernel migrates as a function of the temperature gradient at the particle location. The migration distance is calculated according to the formulation of Reference 23. 


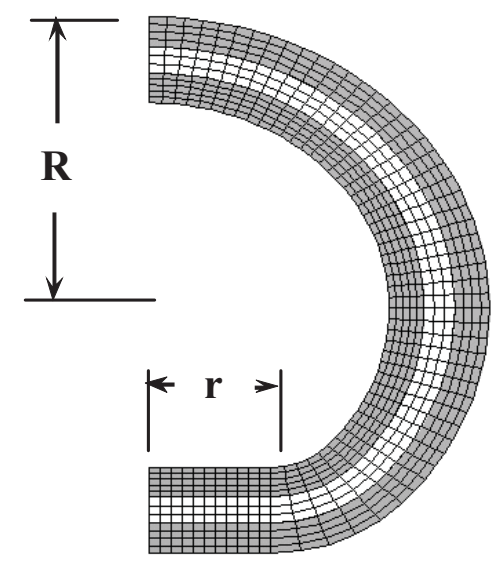

Figure 4-6. Finite element model for an aspherical fuel particle.

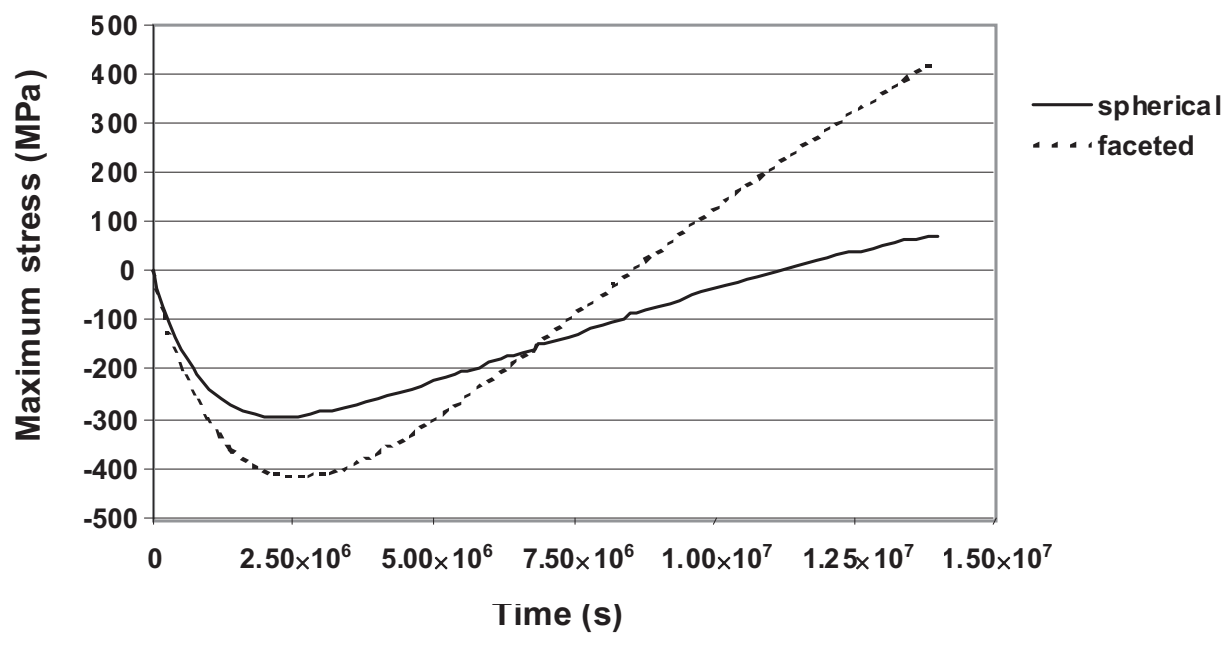

Figure 4-7. Stress histories for a faceted and spherical fuel particle.

Particle failure is assumed to occur when the kernel comes into contact with the $\mathrm{SiC}$ layer. The evaluation of particles for failure due to the amoeba effect is discussed in more detail in Section 5.3.2.4. 


\section{MULTI-DIMENSIONAL STRESS BEHAVIOR}

\subsection{Overview}

Based upon post-irradiation examination results of U.S. fuel particles, it was concluded that modeling one-dimensional pressure vessel failure alone was insufficient. For example, one-dimensional pressure vessel failure models could not predict particle failures due to irradiation-induced shrinkage cracks in the IPyC layer. Therefore, several models to predict multi-dimensional behavior were implemented in PARFUME. For example models which predict particle failure due to asphericity, debonding, and IPyC cracking have been incorporated into PARFUME. A general software development process was followed in order to incorporate these multi-dimensional effects into the code.

As depicted in Figure 5-1, the methodology used to model the effects of multi-dimensional behavior in PARFUME consists of a 3-step process which includes: finite element analyses, statistical analyses, and failure probability determinations. A detailed finite element (FE) analysis on cracked, debonded, or aspherical particles for a selected range of parametric variations is used, in conjunction with results from the solution described in Section 4 to make a statistical approximation of the stress levels in any particle. Using this approach, numerous parameters can be varied statistically (about a mean value) including thicknesses of the three coating layers, densities and Bacon Anisotropy Factor (BAF) values for the pyrocarbons, irradiation temperature, the creep coefficient for the pyrocarbons, kernel diameter, buffer thickness, and Poisson's ratio in creep for the pyrocarbons. In the statistical approach used, ${ }^{24} \mathrm{FE}$ analyses are performed (using the ABAQUS program ${ }^{22}$ ) on just enough cases that the effects of varying each parameter individually can be determined. The information obtained from the FE analyses is used in conjunction with one-dimensional stress analysis results obtained using the closed-form solution described in Section 4. Simple statistical fits are performed on the results of the analyses and a correlation is drawn between the stresses in the one-dimensional and multi-dimensional particles for the same parametric variations. Finally, particle failure probabilities are computed using Weibull statistics.

\subsection{Statistical Approach for Determining Stresses}

The statistical approach used to correlate one-dimensional and multi-dimensional stresses is developed in terms of variations about mean values for the parameters that describe a batch of fuel particles. Since a set of mean values,_and the statistical deviations from the mean for any particular batch of particles is generally unique to that batch, it is important that the method be flexible in addressing any set of mean values and statistical variations about the mean.

The fundamental equation used to determine multi-dimensional stress (i.e., $\sigma_{c}$ ) in particles by correlating mean stress values is shown in Equation (5-1) and consists of four quantities: $\sigma_{c v}, \sigma_{u v}, \sigma_{u}$, and $h$ (see Nomenclature for definitions). When all parameters in a cracked particle in a multi-dimensional stress state are at the mean values for a specified batch of particles, the maximum calculated stress in the SiC layer of the particle (as determined by FE analysis) assumes a value $\sigma_{c v}$. Similarly, when all parameters in a noncracked one-dimensional particle are at the same mean values, the maximum calculated stress in the $\mathrm{SiC}$ layer (as determined by the closed-form PARFUME solution) is $\sigma_{u \bar{v}}$. Upon varying a single parameter $v$ 
where there is a function $h_{i}$ for each statistically varying parameter.

Figure 5-2 demonstrates how the $h$ function for a parameter is produced from several data points. At the midpoint, where $\Delta v=0, h$ always has a value of 1 . The other data points are determined by performing FE analysis in which the parameter under consideration is varied approximately four standard deviations to each side of its mean value. All other parameters in these analyses are held at their mean values. The same cases are solved for a one-dimensional particle using the closed-form solution in PARFUME. The value for $h$ at these data points is the ratio between the stresses for multi-dimensional and one-dimensional particles, respectively. A polynomial curve fit is then made on the data points to generate the quadratic function $h$. Though Figure 5-2 uses five data points to produce the $h$ function, a suitable function can generally be produced from just the two outside data points in addition to the center point (i.e., $\Delta v=0$ )

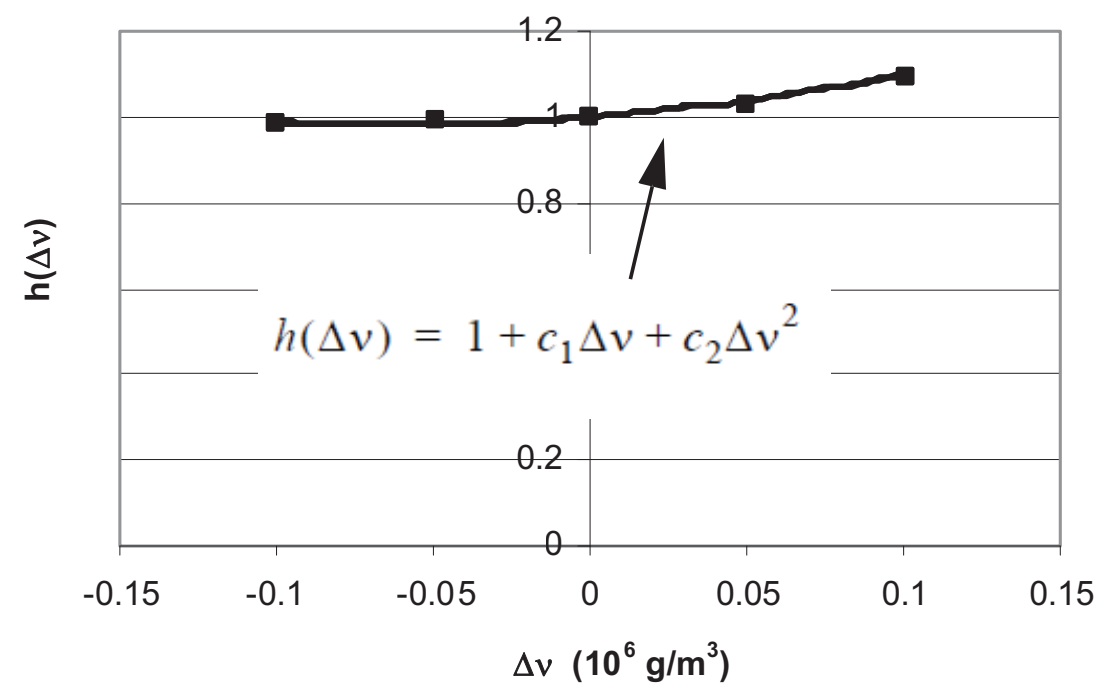

Figure 5-2. Generating an $h$ function for variations in IPyC density.

Determining a function $h$ for each statistically varying parameter in a batch of particles can in some cases involve more work than is desired to calculate a failure probability, since several FE analyses must be performed to generate a set of $h$ functions. For situations where some loss of accuracy in the failure probability is acceptable, it has been found that a reasonable estimate can be obtained by simply setting the correlation functions equal to one. With this approximation, the SiC stress in the multi-dimensional particle becomes:

$\sigma_{c}\left(v_{j}, v_{k}, v_{l}, \ldots\right) \cong \frac{\sigma_{c \bar{v}}}{\sigma_{u \bar{v}}} \sigma_{u}\left(v_{j}, v_{k}, v_{l}, \ldots\right)$

It is also shown in Reference 24 that a high level of accuracy may be attained by including selected $h$ functions in the equation above. Reference 24 shows that including $h$ functions for just the three layer thicknesses gave very accurate results when evaluating particles having a cracked IPyC. The user of PARFUME inputs values for coefficients $c_{1}$ and $c_{2}$ [in Equation (5-2)] for $h$ functions that correspond to the selected parameters, and sets $c_{1}$ and $c_{2}$ equal to zero for all other parameters. 


\subsection{Failure Probability Determination}

\subsubsection{Failure Probability Theory}

Once stresses have been determined, the next step is to use the stresses calculated to determine particle failure probabilities in the fuel performance model. As with other models, Weibull statistics are used to evaluate failure. The methodology is discussed in the context of SiC layer failure, but this process is applicable to any layer.

In this approach, a strength distribution for the $\mathrm{SiC}$ layer is characterized by a mean strength $\sigma_{m s}$ and a modulus $m$. The mean strength for these evaluations is determined from a characteristic strength $\sigma_{0}$ obtained from data of Reference ${ }^{25}$. The method used for determining the mean strength is summarized below.

In the Weibull theory, the failure probability for the $\mathrm{SiC}$ is as follows: ${ }^{26}$

$P_{f}=1-e^{-\int_{V}\left(\sigma / \sigma_{0}\right)^{m} d V}$

Once finite element results are obtained from the analysis of the spherical particle, the stress integration above can be performed using the principle of independent action (PIA) model for treating multiaxial stress states: ${ }^{26}$

$\int_{V} \sigma^{m} d V=\int_{V}\left(\sigma_{1}^{m}+\sigma_{2}^{m}+\sigma_{3}^{m}\right) d V$

Since only tensile stresses contribute to fracture of the material, compressive stresses are not included in the integration. Based on the stress distribution throughout the volume of the SiC layer, the integral above assumes a value that can be written as follows:

$$
\int_{\mathcal{V}} \sigma^{m} d V=\sigma_{c}^{m} \int_{V} f(V) d V=\sigma_{c}^{m} I
$$

where $\sigma_{c}$ is the maximum value calculated for a principal stress anywhere in the volume. The integral $I$ is a normalized integration of the stress distribution, where the maximum stress (taken to the $m$ power) has been factored out. The failure probability then becomes:

$$
P_{f}=1-e^{-\frac{\sigma_{c}^{m}(I)}{\sigma_{0}^{m}}}
$$

In the fuel performance model, the strength of the layer is sampled according to the following cumulative distribution function: ${ }^{27}$

$P_{f}=1-e^{-\left(\sigma_{c} / \sigma_{m s}\right)^{m}}$ 
The mean strength $\sigma_{m s}$ then is determined by applying the condition that the failure probability calculated by the fuel performance model per Equation (5-9) equals that of Equation (5-8). This is done by equating the exponents of the two equations and using stresses for a particle having nominal values for all parameters to determine the integral $I$. The effective mean strength for the layer is then defined to be:

$\sigma_{m s}=\sigma_{0} / I_{n}^{1 / m}$

where the subscript $n$ denotes a particle having nominal values for all parameters. Note that the integral $I_{n}$ for the IPyC layer and for the SiC layer of a one-dimensional spherical particle is calculated within PARFUME, for example when evaluating failure due to pressure stresses (one-dimensional analysis). The integral $I_{n}$ for the $\mathrm{SiC}$ layer of a multi-dimensional particle is based on the integration of the stress distribution in the $\mathrm{SiC}$ obtained from finite element analysis of a particle having nominal values for all parameters. The mean strength from Equation (5-10) for a batch of fuel particles is then input to the PARFUME code so that a failure probability can be determined. In the Monte Carlo routine of PARFUME, the maximum stress $\sigma_{c}$ in the $\mathrm{SiC}$ for each particle is compared to a strength that is sampled from a Weibull distribution having mean strength $\sigma_{m s}$ and modulus $m$ to determine whether or not particle failure occurs.

\subsubsection{Determining Failure Modes}

Understanding and determining failure modes is an important aspect of the internal logic structure in PARFUME. The various failure modes described below are assimilated into PARFUME such that failure of a particle in one mode excludes failure in any other mode for the same particle. The fundamental failure logic is described in Section 5.4.

As discussed in Section 4.6, the multi-dimensional failure mechanisms modeled in PARFUME are failures of the $\mathrm{SiC}$ due to 1) cracking of the IPyC layer, 2) partial debonding of the IPyC from the $\mathrm{SiC}$ layer, 3) aspherical geometry, and 4) the amoeba effect. The treatment of the cracked IPyC is published in References ${ }^{28}$ and ${ }^{29}$, while debonding and asphericity are published in Reference ${ }^{30}$. Details of these treatments are described below.

Another failure mechanism that has been investigated but not yet modeled in the code is failure due to thinning or degradation of the $\mathrm{SiC}$ layer. ${ }^{31}$

\subsubsection{Cracking of the IPyC}

To evaluate particles for potential failure due to a cracked IPyC, the Code first determines whether the IPyC cracks. This is done by calculating the tangential stress in the IPyC layer using the solution in the subroutine FSICFUEL, which is a subroutine that performs all structural mechanics calculations in PARFUME. In the solution schemes discussed in Section 5.4, it compares this tangential stress to a strength that is sampled from a Weibull distribution having mean strength $\sigma_{m s}$ and modulus $m$. The mean strength $\sigma_{m s}$ for the IPyC is calculated using Equation (5-10), where the integral $I_{n}$ accounts for the stress distribution throughout the volume of the IPyC, and $\sigma_{0}$ is a characteristic strength for the IPyC. Because of the symmetry of the stress distribution, one-dimensional theory is appropriate; therefore, this integration is performed internally in the PARFUME code instead of being determined from results of FE analysis. If the tangential stress exceeds the strength for a given particle, then the IPyC layer is assumed to fail. 
If the code determines that the IPyC cracks, it then evaluates the integrity of the SiC layer. Multidimensional behavior generally creates stress concentrations in the $\mathrm{SiC}$ material that can lead to particle failure. These stresses are estimated according to the statistical methodology described above and then used to determine particle failure probabilities in the fuel performance model. As has been historically done in fuel particle evaluations, Weibull statistics are used to evaluate failures of the SiC layer. The mean strength for these evaluations is determined from the characteristic strength $\sigma_{0}$ according to Equation (5$10)$.

To evaluate the structural integrity of the $\mathrm{SiC}$ layer, PARFUME calculates a stress for the $\mathrm{SiC}$ layer utilizing the statistical method described above in Equation (5-3) or Equation (5-4). It compares this stress to a strength that is sampled from a Weibull distribution having mean strength $\sigma_{m s}$ and modulus $m$. The mean strength is calculated from Equation (5-10), where the integral $I_{n}$ represents a stress distribution obtained from an ABAQUS ${ }^{22}$ analysis of a cracked particle having mean values for all parameters. Particle failure is indicated when the maximum stress exceeds the sampled strength.

The characteristic strength for the SiC layer is obtained from the General Atomics data. The integral $I_{n}$ is a normalized integration of the stress distribution over the volume $V$ of the $\mathrm{SiC}$ layer as follows:

$I_{n}=\frac{\int_{v}\left(\sigma_{1}{ }^{m}+\sigma_{2}{ }^{m}+\sigma_{3}{ }^{m}\right) d V}{\sigma_{c}{ }^{m}}$

where the subscript $n$ denotes a particle having mean values for all parameters. Since only tensile stresses contribute to fracture of the material, compressive stresses are not included in the integration. Equation (511) accounts for the stress distribution in the layer resulting from multi-dimensional behavior.

\subsubsection{Partial Debonding of the IPyC from the SiC}

To evaluate particles for potential failure due to debonding, the Code first determines whether debonding occurs. It calculates the radial stress at the interface between the IPyC and $\mathrm{SiC}$ layers using the closedform solution in the subroutine FSICFUEL. It compares this stress to the bond strength between layers. PARFUME currently treats the bond strength as a parameter having a Gaussian distribution with a specified mean value and standard deviation. In the Monte Carlo or integration solution schemes for calculating particle failures, the bond strength for a particle is obtained from this distribution. If the radial stress exceeds the bond strength for a given particle, then debonding is assumed to occur at the IPyC and $\mathrm{SiC}$ interface. If the code determines that debonding occurs, it then calculates a stress for the $\mathrm{SiC}$ layer utilizing the statistical method described above, i.e., Equation (5-3) or Equation (5-4). It compares this stress to a strength that is sampled from a Weibull distribution having mean strength $\sigma_{m s}$ and modulus $m$. The mean strength is calculated from Equation (5-10), where the integral $I_{n}$ [of Equation (5-11)] represents a stress distribution obtained from the ABAQUS analysis of a debonded particle having mean values for all parameters (referred to as a nominal particle). With the moving crack tip, significant stress intensifications may occur at many points along the debonded path. These stress concentrations are typically not as severe as those at the crack tip of a cracked IPyC, but they occur over a larger portion of the $\mathrm{SiC}$ volume. Because the peak stresses at these points do not occur simultaneously, the calculation of $I_{n}$ is based on a stress distribution reflecting the maximum stresses that occur at any time during irradiation.

Note that the radial stress must exceed the bond strength in order to determine the stress distribution in the nominal debonded particle. For example if the maximum radial stress for the nominal particle is less 
than the mean bond strength, then debonding would not occur in the nominal particle. Debonding could, however, still occur in some particles in the batch. In order to capture the debonding of these particles in the calculations, the bond strength (in the ABAQUS analysis of the nominal particle) should be set equal to the maximum radial stress minus a selected value $\varepsilon$, and $I_{n}$ should be calculated from the resulting stress distribution. Note that scoping studies have shown that $5 \mathrm{MPa}$ is a reasonable value to choose for $\varepsilon$.

\subsubsection{Asphericity}

In evaluating asphericity, the Code calculates a maximum stress for the $\mathrm{SiC}$ layer utilizing the statistical method described above [Equation (5-3) or Equation (5-4)]. However, a second term is added to the right hand side of these equations to correctly estimate the maximum stress $\sigma_{c}$ for an aspherical particle ${ }^{30}$, as follows for Equation (5-4):

$$
\sigma_{c}\left(v_{j}, v_{k}, v_{l}, \ldots\right) \cong \frac{\sigma_{c \bar{v}}}{\sigma_{u \bar{v}}} \sigma_{u}\left(v_{j}, v_{k}, v_{l}, \ldots\right)+\frac{\Delta \sigma_{c \bar{v}}}{\Delta \sigma_{u \bar{v}}} \Delta \sigma_{u}\left(v_{j}, v_{k}, v_{l}, \ldots\right)
$$

where $\Delta \sigma_{c v}, \Delta \sigma_{u \bar{v}}$, and $\Delta \sigma_{u}$ are changes in the stresses $\sigma_{c \bar{v}}, \sigma_{u \bar{v}}$, and $\sigma_{u}$ in going from the first extremum (or minimum) to the end of irradiation in each respective stress time history. Refer to Figure 4-7 for a typical time history of the $\mathrm{SiC}$ principal stress in a faceted particle. If a second extremum (or maximum) occurs before the end of irradiation is reached, then $\Delta \sigma_{c v}, \Delta \sigma_{u v}$, and $\Delta \sigma_{u}$ are taken as changes in these stresses in going from the minimum to the maximum. This ensures calculation of the largest value of stress that occurs anytime during the irradiation history. In Equation (5-12), $\sigma_{c \bar{v}}, \sigma_{u \bar{v}}$, and $\sigma_{u}$ are stress values occurring at the time of the minimum in each time history. The first term then takes the solution from time zero to the time of the minimum in the stress history, while the second term takes the solution from the minimum to the end of irradiation or to a maximum, whichever occurs first. The additional term is needed for asphericity evaluations because failures due to asphericity occur after the first extremum for $\sigma_{u}$ has been reached, when shrinkage effects from the pyrocarbons are diminishing.

With the addition of a second term, Equation (5-3) becomes:

$$
\begin{aligned}
& \sigma_{c}\left(v_{j}, v_{k}, v_{l}\right) \cong \frac{\sigma_{c \bar{v}}}{\sigma_{u \bar{v}}} \sigma_{u}\left(v_{j}, v_{k}, v_{l}, \ldots\right) h_{1 j}\left(\Delta v_{j}\right) h_{1 k}\left(\Delta v_{k}\right) h_{1 l}\left(\Delta v_{l}\right) \ldots \\
& +\frac{\Delta \sigma_{c \bar{v}}}{\Delta \sigma_{u \bar{v}}} \Delta \sigma_{u}\left(v_{j}, v_{k}, v_{l}, \ldots\right) h_{2 j}\left(\Delta v_{j}\right) h_{2 k}\left(\Delta v_{k}\right) h_{2 l}\left(\Delta v_{l}\right) \ldots
\end{aligned}
$$

The second set of correlation functions $h_{2 i}$ is determined in the same way as the first set $h_{1 i}$ (Section 5.1), using output from the same ABAQUS finite element analyses.

PARFUME compares the stress from Equation (5-12) or (5-13) to a strength that is sampled from a Weibull distribution having mean strength $\sigma_{m s}$ and modulus $m$. If the stress exceeds the strength for a given particle, then the particle is assumed to have failed. Note that the mean strength is calculated from Equation (5-10), where the integral $I_{n}$ represents a stress distribution obtained from the ABAQUS analysis of a faceted particle having mean values for all parameters (referred to as a nominal particle). If there is no asphericity, then the solution reverts to the traditional pressure vessel failure of a perfectly spherical particle, with an SiC strength appropriate for this symmetrical stress distribution. 


\subsubsection{Amoeba Effect}

Kernel migration (amoeba effect) occurs within the presence of a macroscopic temperature gradient and is calculated according to a standard formulation. ${ }^{23}$ This algorithm utilizes kernel migration coefficients derived from experimental data. For UCO fuel where kernel migration is expected to be miniscule, a General Atomics derived correlation ${ }^{23}$ is used. For $\mathrm{UO}_{2}$ fuel, where kernel migration can be significant,

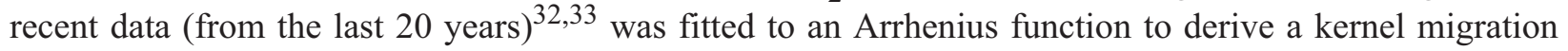
coefficient correlation. Particle failure is assumed to occur when the kernel comes into contact with the SiC layer.

Determining failure of the $\mathrm{SiC}$ due to the amoeba effect is a straightforward process. The distance that the kernel has migrated is calculated at each time step in a particle solution using a kernel migration coefficient (KMC) appropriate for the fuel type. The $\mathrm{KMC}$ is described in Equation (5-14), where $T$ is the kernel temperature $(\mathrm{K}), d y / d t$ is the observed kernel migration rate $(\mathrm{m} / \mathrm{s})$, and $d T / d x(\mathrm{~K} / \mathrm{m})$ is the temperature gradient across the fuel matrix. ${ }^{34}$

$K M C=T^{2} \frac{d y}{d t}\left(\frac{d T}{d x}\right)^{-1}$

Once the kernel has migrated far enough (as determined from integrating $\frac{d y}{d t}$ over time) to make contact with the $\mathrm{SiC}$, the $\mathrm{SiC}$ is assumed to fail.

\subsubsection{Thinning of the SiC Layer}

As stated above, thinning of the $\mathrm{SiC}$ is an additional failure mechanism that is currently under development. Results of a study into the effects of SiC layer thinning are described in Reference ${ }^{31}$. Thinning of the $\mathrm{SiC}$ in local areas makes a negligible contribution to the particle failure probability when considering failures of the $\mathrm{SiC}$ due to stresses in the layer. However, complete penetration through the $\mathrm{SiC}$ provides an open path for fission product transport, and, therefore, constitutes a functional failure of the particle. For thinning over global areas of the $\mathrm{SiC}$, failures can occur due to stress concentrations at the edges of the thinned regions induced by shrinkage of the pyrocarbons. If the thinning occurs over a significant portion (or all) of the $\mathrm{SiC}$ surface, the detachment of the IPyC from the $\mathrm{SiC}$ over the thinned region also enhances the probability that the IPyC will fail due to internal pressure loading. If the IPyC fails, then the internal pressure loading is directly transmitted to a portion or all of the $\mathrm{SiC}$ surface area, which can lead to failure of the $\mathrm{SiC}$ layer. The criterion used in PARFUME for the failure of thinned particles due to internal pressure loading, therefore, will include an evaluation for failure of the IPyC layer.

As part of the $\mathrm{SiC}$ thinning mechanism, a $\mathrm{Pd}-\mathrm{SiC}$ interaction model is nearing completion. To date, all available in-reactor data for Pd penetration in $\mathrm{SiC}^{35,36,37,38}$ have been fitted to an Arrhenius function. The resulting penetration depth correlation will be coupled with finite element stress analyses of corroded, or thinned, $\mathrm{SiC}$ to develop a failure algorithm. 


\subsection{Solution Schemes for Predicting Failure}

PARFUME provides two basic solution schemes for predicting particle failure: Monte Carlo and numerical integration. An advantage of having two solutions schemes is that the integration scheme can be used to check the results of the Monte Carlo scheme and vice versa. The integration scheme includes both a full integration solution scheme and a fast integration solution scheme. Using the fast integration scheme can substantially reduce the time required to perform the integration with only a minimal loss of accuracy. Therefore, an added advantage of the integration method is that it can greatly enhance efficiency of the failure probability calculation. The Monte Carlo and integration failure probability schemes are discussed below.

\subsubsection{Monte Carlo}

In the Monte Carlo routine of PARFUME, stresses are analyzed for a statistically sampled batch of particles, where the parameters sampled may include any of those identified in Section 5.1 together with the strength for each of the coating layers. The stresses in each sampled particle are solved incrementally from the beginning to the end of irradiation. At each time increment, the particle is checked for debonding between the IPyC and SiC layers, for cracking of the IPyC layer, and for failure of the SiC layer. If debonding occurs, the particle is checked for failure of the $\mathrm{SiC}$ in that mode throughout the remainder of irradiation. If the particle does not fail due to debonding, then the particle is checked for failure of the $\mathrm{SiC}$ due to the buildup of internal gas pressure. If cracking of the IPyC occurs, then the particle is checked for failure of the $\mathrm{SiC}$ due to the cracked $\mathrm{IPyC}$ at each increment thereafter. If the particle does not fail due to IPyC cracking, then the particle is checked for failure of the $\mathrm{SiC}$ due to buildup of internal pressure. If neither cracking nor debonding occurs, then the $\mathrm{SiC}$ layer is evaluated for failure due to buildup of internal gas pressure. In addition to these potential failure modes, a particle is evaluated at each time increment for failure due to the amoeba effect.

If the particle is aspherical, then the stress in the $\mathrm{SiC}$ layer due to internal pressure is adjusted to account for the asphericity. Also, the strength of the $\mathrm{SiC}$ layer is based on the stress distribution for an aspherical particle. Failure of the $\mathrm{SiC}$ layer is then measured in terms of this adjusted stress and $\mathrm{SiC}$ strength. If there is no asphericity, then the solution reverts to the traditional pressure vessel failure of a perfectly spherical particle, with an $\mathrm{SiC}$ strength appropriate for this symmetrical stress distribution. PARFUME allows the user to specify statistical distributions for the bond strength and the aspect ratio when considering debonding and asphericity. It also allows the user to opt out from considering debonding or asphericity.

In the failure evaluations, the Weibull strength of the $\mathrm{SiC}$ layer is based on a stress distribution corresponding to a cracked IPyC, a debonded IPyC, or an intact IPyC, whichever is appropriate. In the evaluation for failure due to gas pressure, the effect of any cracking or debonding of the IPyC layer is neglected. This is because the crack or debond has only a minor effect on the overall $\mathrm{SiC}$ stress distribution once the hoop stress in the $\mathrm{SiC}$ becomes tensile.

PARFUME retains the time and location at which each failure occurs in the Monte Carlo sampling process. This information is used to construct a time evolution of the failure probability for a batch of particles. It is also used as input to the fission product diffusion calculations. 


\subsubsection{Integral Formulation}

In the integration approach, the failure probability $P_{f}$ for a particle batch will be formulated in terms of an integral. Because the integral typically involves Gaussian statistical distributions for particle design parameters, it generally cannot be integrated in closed-form. The development of the failure probability integral and methods for numerically evaluating the integral are described below.

\subsubsection{Full Integration}

Failure of a particle is assumed to occur when the maximum stress $\sigma$ in the $\mathrm{SiC}$ layer exceeds the strength $\lambda$. Initially assuming that the strength levels $\lambda$ for the particles in a batch are distributed normally according to a Gaussian distribution, the particles in a batch have a mean strength $\lambda_{s}$ with a standard deviation $D_{s}$. The stress $\sigma$ is a function of a parameter $v_{k}$ that also is assumed to vary according to some statistical distribution. Thus, the stress in a particle can be expressed as follows:

$\sigma=g\left(v_{k}\right)$

Assuming momentarily that the parameter $v_{k}$ is fixed for a batch of particles, then the probability that the stress in a particle exceeds its strength is: ${ }^{39}$

$P_{f}\left(v_{k}\right)=\int_{-\infty}^{g\left(v_{k}\right)} \frac{1}{\sqrt{2 \pi} D_{s}} e^{\frac{-\left(s-\lambda_{s}\right)^{2}}{2 D_{s}^{2}}} d s$

This is the failure probability associated with a specific value for the parameter $v_{k}$. We next consider variations in this parameter. If $a_{i}$ is the fraction of particles within the batch that have a parameter $v_{k}$ equal to $v_{k i}$, then the total failure probability for the batch is:

$P_{f}=a_{1} P_{f}\left(v_{k 1}\right)+a_{2} P_{f}\left(v_{k 2}\right)+a_{3} P_{f}\left(v_{k 3}\right)+\ldots$

This summation is converted to an integral by writing the $a_{i}$ as a density function for the parameter $v_{k}$.

$P_{f}=\int_{-\infty}^{\infty} a\left(v_{k}\right) P_{f}\left(v_{k}\right) d v_{k}$

If the parameter $v_{k}$ is assumed to be distributed normally with a mean value $\mu_{k}$ and a standard deviation of $D_{k}$, then

$P_{f}=\int_{-\infty}^{\infty} \int_{-\infty}^{g\left(v_{k}\right)} \frac{1}{\sqrt{2 \pi} D_{k}} e^{\frac{-\left(v_{k}-\mu_{k}\right)^{2}}{2 D_{k}{ }^{2}}} \frac{1}{\sqrt{2 \pi} D_{s}} e^{\frac{-\left(s-\lambda_{s}\right)^{2}}{2 D_{s}{ }^{2}}} d s d v_{k}$

Determining the failure probability for this batch of particles is reduced to performing the integration above. Because Equation (5-19) must be solved numerically, its accuracy depends on the level of precision in the numerical integration performed. This is discussed later in more detail. 
The formulation above can be extended to situations where the stress $\sigma$ is a function of several parameters that vary according to some statistical distribution. Consider the case of a batch of particles where the stress is a function of two such parameters, $v_{j}$ and $v_{k}$ :

$$
\sigma=g\left(v_{j}, v_{k}\right)
$$

The probability of failure associated with specific values $v_{j}$ and $v_{k}$ for the two parameters is:

$$
P_{f}\left(v_{j}, v_{k}\right)=\int_{-\infty}^{g\left(v_{k}\right)} \frac{1}{\sqrt{2 \pi} D_{s}} e^{\frac{-\left(s-\lambda_{s}\right)^{2}}{2 D_{s}^{2}}} d s
$$

If $a_{m}$ is the fraction of particles having parameter $v_{j}$ equal to $v_{j m}$, and $a_{n}$ is the fraction of particles having parameter $v_{k}$ equal to $v_{k n}$, then the total batch failure probability is:

$P_{f}=\sum_{m} \sum_{n} a_{m} a_{n} P_{f}\left(v_{j m}, v_{k n}\right)$

Again, this is converted to an integral by writing $a_{m}$ and $a_{n}$ as density functions for the parameters $v_{j}$ and $v_{k}$. Then,

$$
P_{f}=\int_{-\infty}^{\infty} \int_{-\infty}^{\infty} a\left(v_{j}\right) a\left(v_{k}\right) P_{f}\left(v_{j,} v_{k}\right) d v_{k} d v_{j}
$$

Assuming that the parameters $v_{j}$ and $v_{k}$ are both normally distributed, then the failure probability becomes:

$P_{f}=\int_{-\infty}^{\infty} \int_{-\infty}^{\infty} \int_{-\infty}^{g\left(v_{j}, v_{k}\right)} \frac{1}{\sqrt{2 \pi} D_{j}} e^{\frac{-\left(v_{j}-\mu_{j}\right)^{2}}{2 D_{j}^{2}}} \frac{1}{\sqrt{2 \pi} D_{k}} e^{\frac{-\left(v_{k}-\mu_{k}\right)^{2}}{2 D_{k}^{2}}} \frac{1}{\sqrt{2 \pi} D_{s}} e^{\frac{-\left(s-\lambda_{s}\right)^{2}}{2 D_{s}^{2}}} d s d v_{k} d v_{j}$

A pattern is established by comparing Equations (5-19) and (5-24). Each parameter considered adds a density function to the integrand and increases the level of integration by one. Any number of parameters can be accommodated in the formulation, but each adds to the computational time required to perform the integration.

In what is labeled the "full integration" method, the limits of integration in the mathematical expression above extend to infinity. In reality, of course, these parameters do not physically cover this range. It is found that a full value for the integral can be attained by integrating over a range of four standard deviations to each side of the mean value for each parameter. An integral for $n$ parameters then appears as follows:

$P_{f}=\frac{1}{\sqrt{2 \pi}^{n+1} D_{j} D_{k} \ldots D_{s}} \int_{\mu_{j}-4 D_{j}}^{\mu_{j}+4 D_{j}} \int_{\mu_{k}-4 D_{k}}^{\mu_{k}+4 D_{k}} \ldots \int_{\lambda_{s}-4 D_{s}}^{g\left(v_{j}, v_{k} \cdots\right)} e^{\frac{-\left(v_{j}-\mu_{j}\right)^{2}-\frac{\left(v_{k}-\mu_{k}\right)^{2}}{2 D_{j}^{2}}}{2 D_{k}{ }^{2}} \ldots \frac{-\left(s-\lambda_{s}\right)^{2}}{2 D_{s}^{2}}} d s d v_{k} d v_{j} \ldots$

Finally, a variation in strengths using Weibull statistics must be considered. Recall that in the Weibull theory, the probability of failure for the particles in a batch is expressed as follows: 
$P_{f}=1-e^{-\left(\frac{\sigma_{\max }}{\sigma_{m s}}\right)^{m}}$

where $\sigma_{\max }$ is the maximum principal stress in the $\mathrm{SiC}$ layer.

We now consider the case where the maximum stress is a function of a parameter $\left(v_{k}\right)$ that varies with some statistical distribution.

$\sigma_{\max }=g\left(v_{k}\right)$

The failure probability associated with a specific value $v_{k}$ for this parameter is:

$P_{f}\left(V_{k}\right)=1-e^{-\left(\frac{g\left(v_{k}\right)}{\sigma_{m s}}\right)^{m}}$

The total failure probability for a batch of particles is:

$P_{f}=\int_{-\infty}^{\infty} a\left(v_{k}\right) P_{f}\left(v_{k}\right) d v_{k}$

where $a\left(v_{k}\right)$ is a density function for the parameter $v_{k}$.

In the situation where the stress is a function of multiple parameters having normal statistical distributions, the total failure probability becomes:

$P_{f}=\frac{1}{\sqrt{2 \pi}^{n} D_{j} D_{k} \ldots} \int_{\mu_{j}-4 D_{j}}^{\mu_{j}+4 D_{j}} \int_{\mu_{k}-4 D_{k}}^{\mu_{k}+4 D_{k}} \ldots e^{-\left(v_{j}-\mu_{j}\right)^{2} / 2 D_{j}{ }^{2}-\left(v_{k}-\mu_{k}\right)^{2} / 2 D_{k}{ }^{2} \ldots}\left[1-e^{-\left(\frac{g\left(v_{j}, v_{k} \ldots\right)}{\sigma_{m s}}\right)^{m}}\right] d v_{k} d v_{j} \ldots$

Unfortunately, Equation (5-30) cannot be integrated in closed form. The traditional method of determining the failure probability with Monte Carlo statistical sampling of the integration variables is a means of evaluating this integral. The other approach exercised in PARFUME is a direct numerical integration of Equation (5-30). The integration is generally performed at eleven Gaussian quadrature points over the range of $\mu_{i}-4 D_{i}$ to $\mu_{i}+4 D_{i}$ for each parameter $v_{l}$. An odd number of points is used so that an integration is always performed at the mean value $\mu_{i}$ for each parameter, which is essential to achieving maximum accuracy in the calculation.

An exception to the use of a Gaussian distribution to characterize the statistical variation in a parameter is in the temperature distribution among particles. The temperature of a fuel pebble, for example, is dependent on its radial position in the pebble. The distribution used to represent the radial density of particles in a pebble is $3 r^{2} / R^{3}$, where $r$ is any radial location in the pebble and $R$ is the outside radius of the pebble. When integrated over the range of 0 to $R$, the density sums to 1 . 
The full integration method is executed in the code with a set of nested DO loops, one for each statistically varying parameter. Each parameter is cycled through its full range of values within its respective loop.

\subsubsection{Fast Integration}

The time required to execute the probability calculation of Equation (5-30) depends on how many parameters are given a statistical variation. For example, the execution time is just a fraction of a second when only one parameter is statistically varied, no matter how small the failure probability. The computation time can, though, become inordinately large when a large number of parameters are considered. Therefore, an approximation is developed below that can substantially reduce the time required to perform the integration with only a minimal loss of accuracy.

Considering for the moment statistical variations in four parameters, Equation (5-30) can be expressed as the following integral:

$$
\begin{aligned}
I_{1,2,3,4} & =\frac{1}{\sqrt{2 \pi}^{4} D_{1} D_{2} D_{3} D_{4}} \int_{-4 D_{4}}^{4 D_{4}} \int_{-4 D_{3}}^{4 D_{3}} \int_{-4 D_{2}}^{4 D_{2}} \int_{-4 D_{1}}^{4 D_{1}} e^{-\Delta v_{1}^{2} / 2 D^{2}-\Delta v_{2}{ }^{2} / 2 D^{2}-\Delta v_{3}^{2} / 2 D^{3}-\Delta v_{4}{ }^{2} / 2 D^{2}} \\
& \times\left[1-e^{-\left(\frac{g\left(\Delta v_{1}, \Delta v_{2}, \Delta v_{3}, \Delta v_{4}\right)}{\sigma_{m s}}\right)^{m}}\right] d \Delta v_{1} d \Delta v_{2} d \Delta v_{3} d \Delta v_{4}
\end{aligned}
$$

or in the basic form:

$I_{1,2,3,4}=\int_{-4 D_{4}}^{4 D_{4}} \int_{-4 D_{3}}^{4 D_{3}} \int_{-4 D_{2}}^{4 D_{2}} \int_{-4 D_{1}}^{4 D_{1}} f\left(\Delta v_{1}, \Delta v_{2}, \Delta v_{3}, \Delta v_{4}\right) d \Delta v_{1} d \Delta v_{2} d \Delta v_{3} d \Delta v_{4}$

where $\Delta v_{i}$ is the variation in parameter $i$ from its mean value $\mu_{i}$. Additionally, the subscript 1,2,3,4 on the integral $I$ indicates that the integral entails variations in the parameters $1,2,3$, and 4 .

The integrand $f\left(\Delta v_{1}, \Delta v_{2}, \Delta v_{3}, \Delta v_{4}\right)$ can be expanded into a Taylor series having the following form:

$$
\begin{aligned}
& f\left(\Delta v_{1}, \Delta v_{2}, \Delta v_{3}, \Delta v_{4}\right)=f(0,0,0,0)\left(1+\sum_{i=1}^{\infty} \sum_{p=1}^{4} a_{i p} \Delta v_{p}{ }^{i}+\sum_{i=1}^{\infty} \sum_{j=1}^{\infty} \sum_{p=1}^{3} \sum_{q=2}^{4} b_{i j p q} \Delta v_{p}{ }^{i} \Delta v_{q}{ }^{j}\right. \\
& +\sum_{i=1}^{\infty} \sum_{j=1}^{\infty} \sum_{k=1}^{\infty} \sum_{p=1}^{2} \sum_{q=2}^{3} \sum_{r=3}^{4} c_{i j k p q r} \Delta v_{p}^{i} \Delta v_{q}^{j} \Delta v_{r}^{k} \\
& \left.+\sum_{i=1}^{\infty} \sum_{j=1}^{\infty} \sum_{k=1}^{\infty} \sum_{l=1}^{\infty} d_{i j k l} \Delta v_{1}^{i} \Delta v_{2}{ }^{j} \Delta v_{3}{ }^{k} \Delta v_{4}^{l}\right) \quad(p<q<r)
\end{aligned}
$$

The coefficients in this series $\left(a_{i p}, b_{i j p q}, c_{i j k p q r}\right.$, and $\left.d_{i j k l}\right)$ are derivatives of the function $f$ with respect to the parameters $v_{i}$. The error that would be incurred in dropping some of the summations in this series may be small, depending on the degree of coupling between the parameters $v_{i}$. The maximum stress 
$g\left(\Delta_{1}, \Delta v_{2}, \Delta v_{3}, \Delta v_{4}\right)$ can similarly be expanded into a Taylor series. A study of the stresses in the coating layers has revealed that $g$ can be well approximated by carrying only terms involving variations in two or fewer parameters. In this case, summations in the series involving variations in three or more parameters can reasonably be neglected. Because the integrand $f$ is strongly a function of the stress $g$, it can be expected that summations in its series involving variations in three or more parameters can be neglected as well.

Prior to eliminating the summations, the following relations can be established from Equations (5-32) and (5-33):

$$
\begin{aligned}
& I=f(0,0,0,0)=1-e^{-\left(\frac{g(0,0,0,0)}{\sigma_{m s}}\right)^{m}} \\
& I_{1}=I\left(1+\int_{-4 D_{1}}^{4 D_{1}} \sum_{i=1}^{\infty} a_{i 1} \Delta v_{1}{ }^{i} d \Delta v_{1}\right)
\end{aligned}
$$

$I_{1,2}=I\left(1+\sum_{p=1}^{2} \int_{-4 D_{p}}^{4 D_{p}} \sum_{i=1}^{\infty} a_{i p} \Delta v_{p}{ }^{i} d \Delta v_{p}+\int_{-4 D_{2}}^{4 D_{2}} \int_{-4 D_{1}}^{4 D_{1}} \sum_{i=1 j=1}^{\infty} \sum_{i j 12}^{\infty} \Delta v_{1}{ }^{i} \Delta v_{2}{ }^{j} d \Delta v_{1} d \Delta v_{2}\right)$

or

$I_{1,2}=I_{1}+I_{2}-I+I \int_{-4 D_{2}}^{4 D_{2}} \int_{-4 D_{1}}^{4 D_{1}} \sum_{i=1 j=1}^{\infty} \sum_{i j 12}^{\infty} b_{i j} \Delta v_{1}^{i} \Delta v_{2}^{j} d \Delta v_{1} d \Delta v_{2}$

Substituting Equation (5-33) into Equation (5-32), neglecting all summations involving more than two parametric variations, and utilizing the relations of Equations (5-34), (5-35), and (5-37), the failure probability integral becomes:

$$
I_{1,2,3,4} \cong I+\sum_{p=1}^{4} I_{p}-4 I+\sum_{p=1}^{3} \sum_{q=2}^{4}\left[I_{p, q}-I_{p}-I_{q}+I\right]
$$

where $p<q$ and only those parameters having a $\Delta v$ that deviates from zero are shown in subscripts. On simplification, 


$$
I_{1,2,3,4} \cong 3 I-2 \sum_{p=1}^{4} I_{p}+\sum_{p=1}^{3} \sum_{q=2}^{4} I_{p, q}
$$

For the more general case of $N$ variables of integration, Equation (5-39) becomes:

$$
I_{1,2, \ldots N} \cong I+\sum_{p=1}^{N} I_{p}-N I+\sum_{p=1}^{N-1} \sum_{q=2}^{N}\left[I_{p, q}-I_{p}-I_{q}+I\right]
$$

which is simplified to

$$
I_{1,2, \ldots N} \cong \frac{(N-2)(N-1)}{2} I-(N-2) \sum_{p=1}^{N} I_{p}+\sum_{p=1}^{N-1} \sum_{q=2}^{N} I_{p, q}
$$

where $p<q$. This resolves the multiple integral (over $N$ variables) into a number of subintegrals that each require integration over two or fewer variables.

There may be some concern that the error associated with neglecting terms involving three or more parametric variations in the Taylor series for $f$ may become significant if a large number of statistically varying parameters are considered. If such error becomes significant, Equation (5-41) can be modified to include the three-level summations [those involving three parametric variations in Equation (5-33)]. To accomplish this, the following relation is needed to complement Equations (5-34) through (5-37):

$$
\begin{aligned}
& I_{1,2,3}=I-\sum_{p=1}^{3} I_{p}+\sum_{p=1}^{2} \sum_{q=2}^{3} I_{p, q} \\
& \quad+\int_{-4 D_{3}}^{4 D_{3}} \int_{-4 D_{2}}^{4 D_{2}} \int_{-4 D_{a}}^{4 D_{a}} \sum_{i=1}^{\infty} \sum_{j=1}^{\infty} \sum_{k=1}^{\infty} c_{i j k 123} \Delta v_{1}{ }^{i} \Delta v_{2}{ }^{j} \Delta v_{3}{ }^{k} d \Delta v_{1} d \Delta v_{2} d \Delta v_{3}
\end{aligned}
$$

Following the same process as before, this leads to the following expression for the multiple integral involving $N$ variables:

$$
\begin{aligned}
I_{1,2, \ldots N} & \cong-\frac{(N-3)(N-2)(N-1)}{6} I+\frac{(N-3)(N-2)}{2} \sum_{p=1}^{N} I_{p} \\
& -(N-3) \sum_{p=1}^{N-1} \sum_{q=2}^{N} I_{p, q}+\sum_{p=1}^{N-2} \sum_{q=2}^{N-1} \sum_{r=3}^{N} I_{p, q, r} \quad(p<q<r)
\end{aligned}
$$

In this case, the full integral is broken into subintegrals that each require integration over three or fewer variables. It is noted that the approximation equations, Equations (5-41) and (5-43), would apply to essentially any multiple integration, not just the failure probability integral for TRISO-coated fuel particles. The accuracy in any case is dependent upon the degree of coupling among the integration variables. The time required to perform the integration using either of Equations (5-41) or (5-43) relative to the full integration can be measured in terms of the number of integration points required for each. It has been found that the integrations for a fuel particle can generally be carried out to a high degree of accuracy in PARFUME 
using 11 integration points over the range of each statistically varying parameter. The number of integration points $n$ needed to perform the full integration of Equation (5-30), then, is:

$n=11^{N}$

Resolving the integral into subintegrals up to level two, as in Equation (5-41), reduces the number of integration points to

$n=1+N(11)+\frac{N(N-1)}{1(2)}(11)^{2}$

whereas the use of Equation (5-43) requires the following number of integration points:

$n=1+N(11)+\frac{N(N-1)}{1(2)}(11)^{2}+\frac{N(N-1)(N-2)}{1(2)(3)}(11)^{3}$

The full integration method is executed in the code with a set of nested DO loops, one for each statistically varying parameter. Each parameter is cycled through its full range of values within its respective loop. The two-level method is executed with two nested loops. These two loops execute subintegrals involving all possible combinations of parameters taken two at a time [the third term on the right-hand side of Equation (5-41)]. They are also used to execute all subintegrals involving variations in one or no parameters [the second and first terms of Equation (5-41) respectively].

Table 5-1 summarizes the number of integration points required for the full, 2-level, and 3-level integration methods for values of $N$ up to 12. It also shows ratios between the number of integration points required for each method, which are a direct measure of the time saved in using the 2-level and 3-level methods. For example, PARFUME would execute a 2-level integration in approximately $1 /\left(4.67 \times 10^{6}\right)$ the time required to execute the full integration when 10 variables are considered. While the 3-level integration method could be implemented if needed, it is not currently employed in PARFUME.

Table 5-1. Number of integration points required for the three integration methods.

\begin{tabular}{|c|c|c|c|c|c|c|}
\hline \multirow{2}{*}{ Number of variables } & \multicolumn{3}{|c|}{ Number of integration points } & \multicolumn{3}{c|}{ Ratios between integration points } \\
\cline { 2 - 7 } & full & two-level & three-level & full/two & full/three & three/two \\
\hline 2 & 121 & 121 & 121 & 1 & 1 & 1 \\
\hline 3 & 1331 & 397 & 1331 & 3.353 & 1 & 3.353 \\
\hline 4 & 14641 & 771 & 6095 & 18.99 & 2.402 & 7.905 \\
\hline 5 & 161051 & 1266 & 14576 & 127.2 & 11.05 & 11.51 \\
\hline 6 & $1.772 \times 10^{6}$ & 1882 & 28502 & 941.3 & 62.16 & 15.14 \\
\hline 7 & $1.949 \times 10^{7}$ & 2619 & 49204 & 7441 & 396.0 & 18.79 \\
\hline 8 & $2.144 \times 10^{8}$ & 3477 & 78013 & 61651 & 2748 & 22.44 \\
\hline 9 & $2.358 \times 10^{9}$ & 4456 & 116260 & 529162 & 20282 & 26.09 \\
\hline
\end{tabular}


Table 5-1. Number of integration points required for the three integration methods. (continued)

\begin{tabular}{|c|c|c|c|c|c|c|}
\hline \multirow{2}{*}{ Number of variables } & \multicolumn{3}{|c|}{ Number of integration points } & \multicolumn{3}{c|}{ Ratios between integration points } \\
\cline { 2 - 7 } & full & two-level & three-level & full/two & full/three & three/two \\
\hline 10 & $2.594 \times 10^{10}$ & 5556 & 165276 & $4.67 \times 10^{6}$ & 156934 & 29.75 \\
\hline 11 & $2.853 \times 10^{11}$ & 6777 & 226392 & $4.21 \times 10^{7}$ & $1.26 \times 10^{6}$ & 33.41 \\
\hline 12 & $3.138 \times 10^{12}$ & 8119 & 300939 & $3.87 \times 10^{8}$ & $1.04 \times 10^{7}$ & 37.07 \\
\hline
\end{tabular}

\subsection{Resolution of Failure Probabilities in the Integration Method}

\subsubsection{Resolution Process}

The integration method is implemented in PARFUME in such a manner that in principle it produces the same failure probability as calculated by the Monte Carlo method. This is done by integrating over a parameter space consisting of those parameters $v$ that exhibit a statistical variation about a mean value. For each differential volume element (of parameter space), PARFUME solves incrementally for stresses in a particle from the beginning to the end of irradiation. It then determines a differential particle failure probability for each of several failure modes (IPyC cracking, partial debonding, internal pressure, and the amoeba effect) and adds this to a cumulative probability for each mode. If a failure probability at the end of a time increment exceeds the accumulated probability at the beginning of the increment, the cumulative probability for the volume element is increased accordingly.

To determine the probability that particles will fail due to IPyC cracking, the code first determines the probability that cracking of the IPyC will occur. It then determines the probability that the SiC layer of a cracked particle will fail due to the presence of the crack. The product of these probabilities then gives a probability for particle failure due to IPyC cracking. The probability that particles will fail due to partial debonding of the IPyC layer is likewise determined by first calculating the probability debonding occurs, then determining the probability that a debonded particle will fail. A complication in these calculations is that there are times when IPyC cracking or IPyC debonding would occur in the same particle, introducing overlap in the probabilities of IPyC cracking and IPyC debonding. To develop an appropriate split between the probabilities of IPyC cracking and IPyC debonding, the code uses the basic process described below. It determines maximum values for the IPyC hoop stress and the radial stress at the interface between IPyC and $\mathrm{SiC}$ layers occurring throughout irradiation. It uses the maximum IPyC hoop stress in Equation (5-9) to determine the probability (within a differential volume element of the parameter space) that the IPyC would crack, where $\sigma_{\max }, \sigma_{m s}$, and $m$ are now maximum stress, Weibull mean strength, and Weibull modulus values for the IPyC layer. Because the bond strength is currently treated in PARFUME as having a normal statistical distribution, the code uses Equation (5-24) to determine the probability that the IPyC layer would debond from the $\mathrm{SiC}$, where $g$ is now the maximum radial stress at the interface and $\lambda_{s}$ is the mean bond strength.

Having determined probabilities that a particle within the differential volume element would crack $\left(P_{a}\right)$ or debond $\left(P_{b}\right)$, the program now determines what fraction of particles within the volume element will crack $(a)$, debond $(b)$, or do neither $(c)$. Two conditions that $a, b$, and $c$ must meet are: 
$a+b+c=1$

$a=\frac{P_{a}}{P_{b}} b$

A third condition comes from recognizing that the probability that a particle neither cracks nor debonds $\left(P_{c}\right)$ is:

$P_{c}=\left(1-P_{a}\right)\left(1-P_{b}\right)$

A particle that would crack may also be a particle that would debond. However, a particle that would do neither has no such overlap. Hence,

$c=P_{c}$

Substituting Equations (5-48), (5-49) and (5-50) into Equation (5-47) gives:

$b=P_{b}\left(1-\frac{P_{a} P_{b}}{P_{a}+P_{b}}\right)$

The fractions $a$ and $b$ are calculated above as if the values for the quantities are developed in a single time step. Since PARFUME solves through irradiation in time increments, the process described above has to be modified accordingly. Within a time increment, the probabilities for cracking and debonding increase incrementally by $\Delta P_{a}$ and $\Delta P_{b}$, respectively, resulting in corresponding increases in the fractions $a$ and $b$ of $\Delta a$ and $\Delta b$. Then Equations (5-47)and (5-48) are modified as follows:

$a+\Delta a+b+\Delta b+c=1$

$\Delta a=\frac{\Delta P_{a}}{\Delta P_{b}} \Delta b$

where $a$ and $b$ are the crack and debond fractions at the beginning of the increment. Equations (5-49) and (5-50) remain unchanged, and the probabilities in these equations are those occurring at the end of the time increment. Substituting Equations (5-49), (5-50), and (5-52) into Equation(5-53) results in:

$\Delta b=\frac{\Delta P_{a}\left(P_{a}+P_{b}-P_{a} P_{b}-a-b\right)}{\Delta P_{a}+\Delta P_{b}}$

The incremental fractions $\Delta a$ and $\Delta b$ are then added to $a$ and $b$, respectively, to produce new failure fractions at the end of the increment.

Once the fractions $a, b$, and $c$ are determined for the volume element, the program computes a differential failure probability for each of three failure mechanisms, i.e. IPyC cracking, IPyC debonding, and internal gas pressure loading. To do this it first calculates the maximum stress in the SiC layer for each of these 
mechanisms in a particle corresponding to the volume element. For IPyC cracking or debonding, this is done using the statistical methodology described in Section 5.2. For internal pressure loading, the maximum stress in the $\mathrm{SiC}$ layer for a spherical particle is calculated using the solution of Section 4. However, if asphericity in the particles is considered, then the maximum SiC stress due to internal pressure is calculated using the methodology described in Section 5.3.2.3. Asphericity can be treated as a statistical parameter, in which case the aspect ratio becomes one of the parameters in Equations (5-12) or (5-13).

For each of the three failure mechanisms, the program then applies the maximum $\mathrm{SiC}$ stress in the integrand of Equation (5-30) to calculate a differential failure probability. It applies the factors $a$ and $b$ to the probabilities for IPyC cracking and debonding, respectively. It applies the following factor to the failure probability for internal pressure loading:

$c+a e^{-\left(\frac{\sigma_{\operatorname{maxa}}}{\sigma_{m s a}}\right)^{m}}+b e^{-\left(\frac{\sigma_{\operatorname{maxb}}}{\sigma_{m s b}}\right)^{m}}$

where,

$\sigma_{\text {maxa }}=$ maximum principal stress in the $\mathrm{SiC}$ layer of a particle having a cracked IPyC (MPa)

$\sigma_{m s a}=$ mean strength for the $\mathrm{SiC}$ layer of a particle having a cracked IPyC $(\mathrm{MPa})$

$\sigma_{\max b}=$ maximum principal stress in the $\mathrm{SiC}$ layer of a particle having a partially debonded IPyC $(\mathrm{MPa})$

$\sigma_{m s b}=$ mean strength for the $\mathrm{SiC}$ layer of a particle having a partially debonded IPyC (MPa)

$m=$ Weibull modulus for the $\mathrm{SiC}$

The second and third terms in this factor allow a particle that survives either IPyC cracking or debonding to subsequently fail due to internal pressure loading. Finally, PARFUME accumulates total failure probabilities for each of the three failure modes utilizing Equation (5-30).

If failure due to the amoeba effect is found to occur in a differential volume element during any time increment, then $100 \%$ of the differential failure probability for the element is attributed to the amoeba effect for that increment.

\subsubsection{Accumulation of Failure Probabilities Through Time}

In executing the integration method, PARFUME accumulates a failure probability for each type of failure mechanism considered (IPyC cracking, failure of the $\mathrm{SiC}$ due to IPyC cracking, etc.). In doing this, it calculates an incremental failure probability for each time increment in the analysis of each particle. The incremental probability for a time increment in a particle is determined by subtracting the probability for that failure mechanism at the beginning of the increment from the probability at the end of the increment. The incremental probability for that time increment for that particle is weighted by the appropriate probability density functions for that particle, then is added to a cumulative probability for that time increment. At the end of the integration, then, each time increment has a cumulative failure probability for that failure mechanism. The cumulative failure probabilities for all increments up to a time $t_{i}$ are then summed to produce a total failure probability at time $t_{i}$. 
If failure due to the amoeba effect is found to occur in the analysis of a particle during any time increment, then the incremental probability for that increment is set to one and is attributed fully to the amoeba effect. This incremental probability is weighted by the probability density functions for that particle, then is added to the cumulative failure probability due to the amoeba effect for that time increment.

Figure 5-3 shows a time history calculated for a representative experiment. The failure probability reaches $2.5 \times 10^{-5}$ early during irradiation due to cracking of the IPyC, then levels off for a period of time. After the neutron fluence reaches $3 \times 10^{25} \mathrm{n} / \mathrm{m}^{2}(\mathrm{E}>0.18 \mathrm{MeV})$, particles progressively fail due to the buildup of internal pressure until the failure probability reaches $1.8 \times 10^{-2}$ at the end of irradiation.

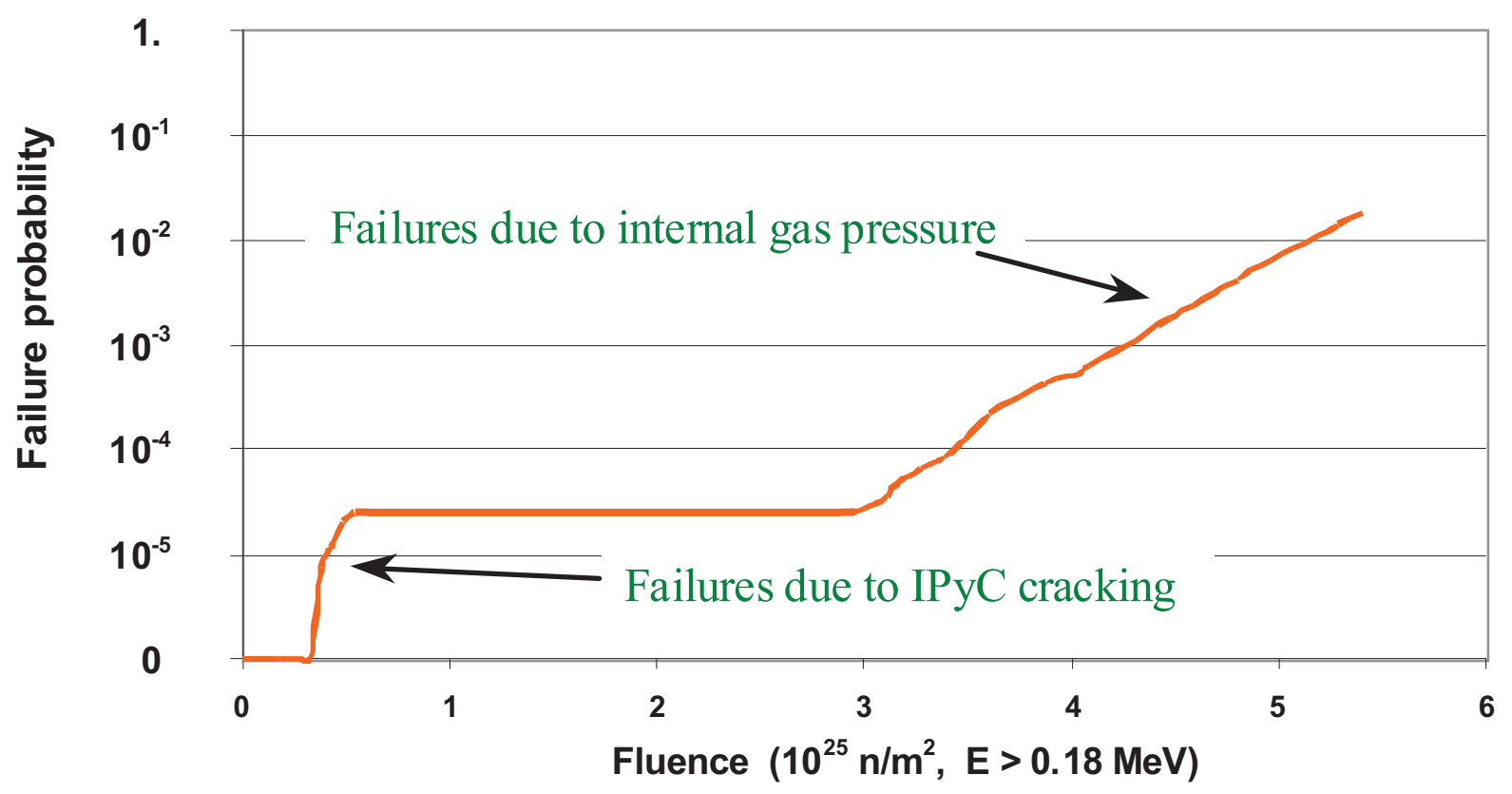

Figure 5-3. Calculated failure probability time history.

\subsection{Linking Particle Failures with the Diffusion Calculations}

\subsubsection{Monte Carlo}

When the Monte Carlo approach is used in the PARFUME program, it is a rather straightforward process to link particle failures to the diffusion calculations. For any sampled particle, normal diffusion is assumed to occur through intact particle layers unless the failure of a coating layer occurs. The strengths of the three coating layers are randomly sampled for each particle, so these are known quantities for each particle analyzed. Additionally, the specific location of the particle within the fuel matrix is known, since this is also determined in the sampling process. The cracking of a pyrocarbon layer or failure of the SiC layer of a particle occurs if the stress in that layer exceeds its strength. Failures are then determined according to the process described in Section 5.4.1. Since the location and timing of any coating layer failure becomes known at the moment it occurs during the solution, diffusivities for the coating layers are immediately 
adjusted and the source term at that location in the fuel matrix is also modified to reflect the failure. The calculated diffusion for all sampled particles is accumulated into a sum that represents the entire fuel matrix.

\subsubsection{Full Numerical Integration}

When the numerical integration method is used to calculate failure probabilities, Equation (5-30) must be modified to enable linking particle failures to the diffusion calculations. With each evaluation of the integrand in Equation (5-30), values are established for all of the integration parameters. This set of parameters is used to calculate a stress history for a particle having this set of parameters. While the integration process according to Equation (5-30) produces an incremental failure probability associated with that set of parameters and the resulting stress history, it does not delineate the time at which failures occur. This timing is needed, though, to correctly link the failures to the diffusion calculation. To make this link, specific values must be established for the strengths of the coating layers so that the time at which the stress reaches the layer strength (i.e., failure occurs) can be determined. This is achieved by differentiating the expression for failure probability [within brackets on the right-hand side of Equation (5-30)] with respect to the stress $s$ and integrating from 0 to the stress level $g\left(v_{j}, v_{k}, \ldots\right)$. This adds another variable to the integral as follows:

$=A \int_{\mu_{j}-4 D_{j}}^{\mu_{j}+4 D_{j}} \int_{\mu_{k}-4 D_{k}}^{\mu_{k}+4 D_{k}} \ldots \int_{0}^{g\left(v_{j}, v_{k} \ldots\right)} e^{-\frac{\left(v_{j}-\mu_{j}\right)^{2}}{2 D_{j}^{2}}-\frac{\left(v_{k}-\mu_{k}\right)^{2}}{2 D_{k}^{2}} \ldots}\left(\frac{m}{\left(\sigma_{m s}\right)^{m}} s^{m-1} e^{-\left(\frac{s}{\sigma_{m s}}\right)^{m}}\right) d s \ldots d v_{k} d v_{j}$

where

$$
A=\frac{1}{\sqrt{2 \pi}^{n} D_{j} D_{k} \cdots}
$$

As with Equation (5-24) the stress $s$ within the integral of Equation (5-56) becomes the strength variable for the coating layer, except that the strength has a Weibull distribution rather than a Gauss distribution. Integrating the strength variable over the range of 0 to $g$ requires dividing this integration region into intervals. The strength value occurring at the midpoint of an interval represents the strength for that interval. Thus, in addition to the set of values for all other parameters we now have a specific value for the coating layer strength for each evaluation of the integrand of Equation (5-56). This enables a determination of the time at which a coating layer failure occurs. The failure probability density associated with an interval is obtained by antidifferentiation of the bracketed quantity in the integrand of Equation (5-56) and application of integration limits at the left $(L)$ and right $(R)$ ends of the interval, which gives:

$P_{D}=e^{-\left(\frac{\sigma_{L}}{\sigma_{m s}}\right)^{m}}-e^{-\left(\frac{\sigma_{R}}{\sigma_{m s}}\right)^{m}}$

This density must be applied to the fission product diffusion calculation for the particle represented by the set of parameters under consideration (including coating layer strength) to properly weight the diffusion calculation. If the coating layer strength for a particle is greater than $g\left(v_{j}, v_{k}, \ldots\right)$, then the density

$P_{D}=e^{-\left(\frac{g\left(v_{j}, v_{k}, \ldots\right)}{\sigma_{m s}}\right)^{m}}$ 
must be applied to the diffusion calculation for a particle where the coating layer does not fail. The density of Equation (5-59) is the probability of survival for the coating layer. These densities are applied in addition to the appropriate densities for the other integration variables.

The discussion above demonstrates the process of resolving the Weibull expression for failure probability into a strength distribution for a coating layer that can then be used to establish the timing of coating layer failures. Since fission product diffusion is affected by failure in any of the three coating layers, this process must be exercised for all three layers. Thus, the analysis of fission product diffusion using the integration scheme adds three integration variables to the overall integral.

\subsubsection{Fast Numerical Integration}

As stated above, incorporating particle failures into the diffusion calculations with the full integration method entails the addition of three integration loops, which significantly increases the computational time required to perform the integration. Therefore, the fast integration approach has also been adapted to calculating failure probabilities when diffusion calculations are performed. The method currently used in PARFUME when diffusion calculations are performed with fast integration is to add two DO loops that are devoted specifically to IPyC and $\mathrm{SiC}$ strength variables. This assures that subintegrals are evaluated that entail variations in two non-strength parameters in conjunction with these strength variables. The OPyC strength variable is treated in the original two loops along with the other statistically varying parameters. OPyC failures generally occur much less frequently than IPyC failures, so need not be captured to the same level of accuracy. With this approach, subintegrals are evaluated that entail variations in one non-strength parameter in conjunction with the $\mathrm{OPyC}$ strength variable.

A fourth strength variable considered in PARFUME is the IPyC/SiC bond strength. Unlike the IPyC, $\mathrm{SiC}$, and $\mathrm{OPyC}$ strength variables, the bond strength is treated with a Gaussian distribution rather than a Weibull distribution. This is done because there is not sufficient bond strength data to identify the parameters of a Weibull distribution or to demonstrate that a Weibull distribution is applicable. In fact, users may often elect to give the same of value of bond strength to all particles in a batch rather than treat it with a statistical distribution. When the bond strength is given a statistical distribution, PARFUME treats it as an integration variable whether or not a diffusion calculation is performed. This is because a Gaussian distribution, unlike a Weibull distribution, has to be integrated numerically.

As with the other strength variables, the integration region for bond strength is divided into uniformly spaced intervals up to the stress level $g\left(\Delta v_{j}, \Delta v_{k} \ldots\right)$. In this case $g\left(\Delta v_{j}, \Delta v_{k}, \ldots\right)$ is the maximum radial (contact) stress between the IPyC and SiC layers that occurs anytime in the irradiation history. The bond will fail for all of these intervals at some time during irradiation. The final integration interval extends from $g\left(\Delta v_{j}, \Delta v_{k} \ldots\right)$ to infinity, and a particle having a bond strength anywhere in this interval will not debond during irradiation. As with the OPyC strength, the bond strength is treated along with the nonstrength variables in the first two DO loops of the fast integration method. This saves significantly in the execution time for the code, and has been shown to produce accurate results in determining probabilities of debonding and failure of the $\mathrm{SiC}$ due to debonding. 


\section{FISSION PRODUCT DIFFUSION MODELS}

Predicting fission product transport by diffusion is necessary for reactor safety and source term assessments. PARFUME allows simulation of fission product (FP) transport from individual TRISO-coated fuel particles to the surrounding graphite and from the surrounding graphite to a release from the fuel element. These capabilities are based on portions of existing coding extracted from the Tritium Migration Analysis Program, TMAP $4^{40,41}$ and adapted to run within PARFUME. Additionally, development is underway on an analytical diffusion model.

A model accounting for release of short-lived fission product gases from failed particles and from uranium contamination in the fuel matrix material is incorporated into PARFUME. This correlation calculates release rate to birth rate $(\mathrm{R} / \mathrm{B})$ ratios for several prominent fission product nuclides. Also based upon the Booth equivalent sphere gas release model ${ }^{42}$, this correlation uses different reduced diffusion coefficients for release from failed particles ${ }^{43}$ and from uranium contamination. ${ }^{45}$

\subsection{TMAP}

TMAP4, a finite difference based code, was originally developed to assist in evaluation of tritium losses from fusion reactor systems during normal and accident conditions. However, it became evident that TMAP4 has application to a much wider variety of problems, including fuel particle analysis. TMAP4 incorporates a one-dimensional diffusion capability that predicts the thermal response of structures (i.e., fuel particles), and solves equations for gas movement through surfaces (layer interfaces) and in bulk materials (i.e., layers). Specifically TMAP4 calculates:

- Movement of species across structure surfaces, governed by dissociation/recombination or by a solution law, such as Sieverts' or Henry's Law.

- Movement by Fick's diffusion law in the bulk of a structure.

- Thermal response of structures to applied heat or boundary temperature loading conditions.

- Chemical reactions within volumes.

- Convective flow between volumes.

Note that TMAP4 was developed for a plate geometry. The corresponding models to simulate movement of FP species across material layer interfaces and through bulk materials was extracted from TMAP4 and modified for use in PARFUME. In addition, those models were modified within PARFUME to treat diffusion within cylindrical and spherical coordinate systems (associated with prismatic and pebblebed reactor geometries).

Similar to previously discussed thermal analyses see Section 3, both "micro" and "macro" diffusion analyses are performed. In this case, FP transport first involves diffusion calculations from individual fuel particles (or the "micro" analysis). Results from each fuel particle then serve as time- and position-dependent FP "sources" for the subsequent fuel element transport analysis (or the "macro" analysis). Details regarding the implementation of fuel particle and fuel element FP transport models in PARFUME are provided in Sections 6.2 and 6.3, respectively. 


\subsection{Micro Solution}

FP transport for fuel particles, or the micro solution, is based on a model with five different materials (the kernel, buffer, IPyC, SiC, and OPyC). It is assumed that FPs flow without resistance through the buffer-to-IPyC gap, if one develops during irradiation. Nodalization of the five remaining materials is handled automatically within the code. Specifically, a node is placed on the inner and outer surface of each material layer. Each layer is then divided into a number of elements of uniform thickness where the thickness is as close to $5 \mu \mathrm{m}$ as possible. Interior nodes are then placed at the center of each element. This nodalization scheme is established at time $=0$ for each particle calculation and dimensions are based on user input plus the statistical variations that may be considered for each particle. There are no changes in nodalization corresponding with changes in particle geometry that may develop during irradiation.

The model for FP diffusion in plane geometry was derived from the general equation for the conservation of atoms. Specifically, the change in the atom concentration (of any specie of interest) can be determined through the application of an atom balance (for the specie). The corresponding equation is given by

$\frac{\partial C}{\partial t}=-\nabla J+S$

The diffusive atom flux (for any specie of interest) is

$J=-D\left(\nabla C+\frac{Q^{\prime} C}{R T^{2}} \nabla T\right)$

The required derivatives for the numerical solution of Equation (6-1) are based on a space-centered, implicit difference approximation for the nodalization scheme shown in Figure 6-1. Specifically, Equations (6-1) and (6-2) can be combined to give

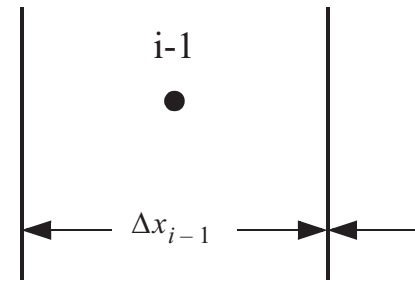

a

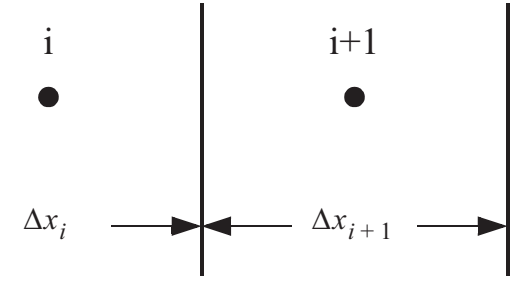

b

Figure 6-1. Finite difference notation used in the numerical diffusion model.

$$
\begin{aligned}
& \frac{C_{i}-C_{i}^{o}}{\Delta t}=\frac{1}{\Delta x}\left[\left\{\left(\frac{D_{i+1}+D_{i}}{\Delta x_{i+1}+\Delta x_{i}}\right)+\left(\frac{\left(Q_{i+1}^{\prime}+Q_{i}^{\prime}\right)\left(T_{i+1}-T_{i}\right)}{R\left(T_{i+1}+T_{i}\right)^{2}}\right)\right\}\left(C_{i+1}-C_{i}\right)\right. \\
& \left.+\left\{\left(\frac{D_{i-1}+D_{i}}{\Delta x_{i-1}+\Delta x_{i}}\right)+\left(\frac{\left(Q_{i-1}^{\prime}+Q_{i}^{\prime}\right)\left(T_{i-1}-T_{i}\right)}{R\left(T_{i-1}+T_{i}\right)^{2}}\right)\right\}\left(C_{i-1}-C_{i}\right)\right]+S
\end{aligned}
$$


where

$x=$ the spatial coordinate $(\mathrm{m})$,

and the ' $O$ ' superscript refers to results from the previous time step.

It should be noted that current time step concentrations $\left(C_{i-1}, C_{i}\right.$, and $\left.C_{i+1}\right)$ are the only unknowns in Equation (6-3). As such, the equation can easily be rewritten in tridiagonal form by collecting terms relative to the unknown concentrations. A series of such equations leads to a tridiagonal matrix, which is solved via Gaussian elimination within PARFUME.

Extensions of the existing numerical model were needed to also allow simulation of fission product diffusion in both cylindrical and spherical coordinates. The spherical coordinate extension should increase fidelity when modeling TRISO-coated fuel particles and pebble bed reactors. The cylindrical coordinate extension should increase fidelity when modeling prismatic reactors.

In cylindrical coordinates, Equations (6-1) and (6-3) become

$\frac{\partial C}{\partial t}=-\frac{1}{r} \nabla(r J)+S$

and

$$
\begin{aligned}
& \frac{C_{i}-C_{i}^{o}}{\Delta t}=\frac{1}{r_{i} \Delta r}\left[\left(\frac{r_{i+1}+r_{i}}{2}\right)\left\{\left(\frac{D_{i+1}+D_{i}}{\Delta x_{i+1}+\Delta x_{i}}\right)+\left(\frac{\left(Q_{i+1}^{\prime}+Q_{i}^{\prime}\right)\left(T_{i+1}-T_{i}\right)}{R\left(T_{i+1}+T_{i}\right)^{2}}\right)\right\}\left(C_{i+1}-C_{i}\right)\right. \\
& \left.+\left(\frac{r_{i-1}+r_{i}}{2}\right)\left\{\left(\frac{D_{i-1}+D_{i}}{\Delta x_{i-1}+\Delta x_{i}}\right)+\left(\frac{\left(Q_{i-1}^{\prime}+Q_{i}^{\prime}\right)\left(T_{i-1}-T_{i}\right)}{R\left(T_{i-1}+T_{i}\right)^{2}}\right)\right\}\left(C_{i-1}-C_{i}\right)\right]+S
\end{aligned}
$$

where $r=$ the spatial coordinate $(\mathrm{m})$.

In spherical coordinates, Equations (6-1) and (6-3) become

$$
\frac{\partial C}{\partial t}=-\frac{1}{r^{2}} \nabla\left(r^{2} J\right)+S
$$

and

$$
\begin{aligned}
& \frac{C_{i}-C_{i}^{o}}{\Delta t}=\frac{1}{r_{i}^{2} \Delta r}\left[\left(\frac{r_{i+1}+r_{i}}{2}\right)^{2}\left\{\left(\frac{D_{i+1}+D_{i}}{\Delta x_{i+1}+\Delta x_{i}}\right)+\left(\frac{\left(Q_{i+1}^{\prime}+Q_{i}^{\prime}\right)\left(T_{i+1}-T_{i}\right)}{R\left(T_{i+1}+T_{i}\right)^{2}}\right)\right\}\left(C_{i+1}-C_{i}\right)\right. \\
& \left.+\left(\frac{r_{i-1}+r_{i}}{2}\right)^{2}\left\{\left(\frac{D_{i-1}+D_{i}}{\Delta x_{i-1}+\Delta x_{i}}\right)+\left(\frac{\left(Q_{i-1}^{\prime}+Q_{i}^{\prime}\right)\left(T_{i-1}-T_{i}\right)}{R\left(T_{i-1}+T_{i}\right)^{2}}\right)\right\}\left(C_{i-1}-C_{i}\right)\right]+S
\end{aligned}
$$

A simplification is evident through examination of Equations (6-3), (6-5), and (6-7). Specifically, if $x$ is taken to be a general spatial variable, the equations can be combined to give 


$$
\begin{aligned}
& \frac{C_{i}-C_{i}^{o}}{\Delta t}=\frac{1}{x_{i}^{n} \Delta x}\left[\left(\frac{x_{i+1}+x_{i}}{2}\right)^{n}\left\{\left(\frac{D_{i+1}+D_{i}}{\Delta x_{i+1}+\Delta x_{i}}\right)+\left(\frac{\left(Q_{i+1}^{\prime}+Q_{i}^{\prime}\right)\left(T_{i+1}-T_{i}\right)}{R\left(T_{i+1}+T_{i}\right)^{2}}\right)\right\}\left(C_{i+1}-C_{i}\right)\right. \\
& \left.+\left(\frac{x_{i-1}+x_{i}}{2}\right)^{n}\left\{\left(\frac{D_{i-1}+D_{i}}{\Delta x_{i-1}+\Delta x_{i}}\right)+\left(\frac{\left(Q_{i-1}^{\prime}+Q_{i}^{\prime}\right)\left(T_{i-1}-T_{i}\right)}{R\left(T_{i-1}+T_{i}\right)^{2}}\right)\right\}\left(C_{i-1}-C_{i}\right)\right]+S
\end{aligned}
$$

where

$$
\begin{aligned}
n \text { (the spatial exponent }) & =0 \text { for plane geometry, } \\
& =1 \text { for cylindrical geometry, } \\
& =2 \text { for spherical geometry. }
\end{aligned}
$$

In PARFUME, user input provides all of the information needed to automatically assign values for the spatial exponent $n$. Specifically, $n$ is always set equal to 2 when simulating diffusion through TRISOcoated fuel particles. Diffusion through the fuel matrix corresponds with the reactor type, which is a required user input. Currently supported options include 'PEBBLEBED', resulting in $n=2$; 'PRISMATIC', resulting in $n=1$; and 'PLANEGEOM', resulting in $n=0$.

The radial position (within the fuel element) of the fuel particle being analyzed is determined at the beginning of each particle FP transport simulation. This ultimately allows development of a positiondependent FP "source" to be used in fuel element transport analyses. During each time step throughout the particle simulation, calculated release fractions are used with the incremental FP yield to assign the initial deposition of FPs from the analyzed particle into the fuel element. FP contamination is assumed to begin diffusion from the outer edge of the buffer.

FP transport calculations assume a "non-flow" condition at the fuel particle center (due to symmetry) and a fixed concentration of FPs of " 0 " on the fuel particle outer surface (i.e., the OPyC outer surface). Use of a fixed outer surface concentration (of essentially any arbitrary value) is recommended to determine the net integrated flow of FPs into or out of a material segment. Calculation of the net integrated flow of FPs is needed as the "source" input for subsequent fuel element transport analyses. Although trapping models, used to account for species trapping due to microstructure irregularities, are implemented in PARFUME, current calculations use diffusivities that effectively combine all transport processes for analysis using a simplified diffusion approach.

FP transport calculations are first completed for "nominal" fuel particles (i.e., those fuel particles that normally remain intact) to establish a "baseline" for the FP "source" used in fuel element transport analyses. Subsequent calculations for all other fuel particles are then completed to add the effects of particle failures to the "baseline". Specifically, provisions are included to allow incremental failures of the 3 outer most layers (i.e., the IPyC, SiC, and $\mathrm{OPyC}$ ). Diffusivities are increased to an arbitrarily large value (currently $1 \times 10^{-6}$ ) in any layer that has been predicted to fail. FP transport from each failed particle that is above the "baseline" is then added to complete the time- and position-dependent "source" for FP transport from fuel elements. 


\subsection{Macro Solution}

Fuel element FP transport, or the macro solution, is based on a model with two different materials: the graphite containing fuel particles and the surrounding graphite without fuel particles. User input defines the dimensions and the number of nodes in each. As previously indicated, PARFUME thermal models calculate time-dependent temperatures for the nodes based on user-specified boundary conditions and the total particle heat load.

The time- and position-dependent FP "source" established during fuel particle analyses is augmented with FP contributions due to uranium contamination. Specifically, uranium contamination (as specified through user input) is initially assumed to be uniformly distributed on the outer surface of OPyC layer throughout the fueled region of the fuel element model. Addition of FPs to the fuel particle FP "source" are based on the level of contamination, the FP yield, and the calculated release fraction.

Using Equation (6-8), FP transport calculations then proceed assuming a "non-flow" condition of FPs at the fuel element centerline (due to symmetry) and a fixed concentration of FPs of " 0 " on the fuel element outer surface (i.e., the outer surface of the unfueled graphite). Use of a fixed outer surface concentration (of essentially any arbitrary value) is the approach recommended to determine the net integrated flow of FPs into or out of a material segment. Calculation of the net integrated flow of FPs is needed to quantify the fuel element FP release. The calculations use diffusivities that effectively combine all transport processes for analysis using a simplified diffusion approach. 


\section{MATERIAL PROPERTIES}

Material properties are needed to support the three primary models contained in the PARFUME code, i.e. the mechanical, thermal, and diffusion models. A discussion of each model including capabilities and limitations is included below.

\subsection{Mechanical}

The mechanical properties used in the stress analysis of fuel particles in PARFUME were obtained from a report compiled by the CEGA Corporation ${ }^{25}$. These properties include elastic moduli and Poisson's ratios, the creep coefficient and Poisson's ratio in creep for the PyC layers, swelling strain rate data for the PyC layers, Weibull statistical parameters, and coefficients of thermal expansion. With the exception of the swelling strain data, the material properties are incorporated into PARFUME with a straightforward application of equations given in Reference 25 .

A linear viscoelastic material model and Weibull statistical strength theory are used to predict material behavior and failure strengths for the buffer layer, PyC layers, and SiC layer. The strain components modeled include thermal, elastic, irradiation-induced swelling, and irradiation-induced steady-state creep.

\subsubsection{Assumptions and Approximations}

Due to uncertainties or incompleteness in the material data, several assumptions and approximations were required to develop the material properties ${ }^{25}$. Additionally, the majority of the material properties were obtained from strip samples (i.e., flat geometry). Though the coating layers in the fuel particle have spherical geometry, it is assumed that these material properties of $\mathrm{PyC}$ and $\mathrm{SiC}$ are representative. Additional details, related to mechanical properties, are presented here:

- In general, application of the material models is limited to the range of $4 \times 10^{25} \mathrm{n} / \mathrm{m}^{2}, \mathrm{E}>0.18$ $\mathrm{MeV}$ ) for fast neutron fluence and $600^{\circ}$ to $1300^{\circ} \mathrm{C}$ for irradiation temperature.

- The densities of the dense structural PyC and the porous PyC are limited to 1.8 to $2.0 \mathrm{Mg} / \mathrm{m}^{3}$ and approximately 0.9 to $1.1 \mathrm{Mg} / \mathrm{m} 3$, respectively; the density of $\mathrm{SiC}$ is at least $3.18 \mathrm{Mg} / \mathrm{m}^{3}$.

- Due to the scarcity of material data, it is assumed in the material models that the effects of several parameters (i.e., temperature, fluence, density) are mutually independent and their combined effect can be obtained by multiplying them together. For example, the modulus of elasticity for dense $\mathrm{PyC}$ is approximated as a product of several effects as shown in Equation (7-1).

- Irradiation-induced creep in the pyrocarbon layers is treated as secondary creep, i.e. the creep strain rate is proportional to the level of stress in the pyrocarbon. The creep coefficient increases significantly with increases in the irradiation temperature.

- Thermal expansion of the pyrocarbons is anisotropic, and is a function of the Bacon Anisotropy Factor (BAF) and temperature.

- Swelling in the pyrocarbon layers is anisotropic and a function of four variables, i.e. fluence level, pyrocarbon density, degree of anisotropy (as measured by BAF), and irradiation temperature. The magnitude of the shrinkage increases as BAF increases or as the irradiation temperature increases. 
- The SiC layer has an elastic modulus (stiffness) that is an order of magnitude higher than that for the pyrocarbons.

- The dependence of Poisson ratio, v, (for the PyC layers) on density, crystallite size, temperature and fluence is largely unknown; therefore, it is assumed that Poisson's ratio is independent of these parameters.

- Most available mechanical property test data for PyC correspond to densities of $1.5 \mathrm{Mg} / \mathrm{m}^{3}$ or higher. For the buffer layer (porous PyC), which has a density of approximately $1.0 \mathrm{Mg} / \mathrm{m}^{3}$, a large extrapolation of the data is required, which may significantly affect its accuracy.

\subsubsection{Modulus of Elasticity}

\subsubsection{Pyrocarbons (IPyC and OPyC layers)}

As shown in Equation (7-1), the elastic modulus for the PyC layers is applied as a function of four variables, i.e. fluence level $(\phi)$, pyrocarbon density $(\rho)$, degree of anisotropy as measured by the BAF, and irradiation temperature $(T)$.

$E\left(\rho, B A F_{o}, L_{c}, \phi, T\right)=E_{o} \cdot E(\rho) \cdot E\left(B A F_{o}\right) \cdot E\left(L_{c}\right) \cdot E(\phi) \cdot E(T)$

The final equations used in PARFUME for elastic modulus in the tangential and radial directions, respectively, are as follows:

$$
\begin{aligned}
& E_{t}=E_{01}(0.384+0.324 \rho)(0.481+0.519 B A F)\left(2.985-0.0662 L_{c}\right)(1+0.23 \phi)(1+0.00015 T) \\
& E_{r}=E_{03}(0.384+0.324 \rho)(1.463-0.463 B A F)\left(2.985-0.0662 L_{c}\right)(1+0.23 \phi)(1+0.00015 T)
\end{aligned}
$$

where $\rho=$ density $\left(\mathrm{Mg} / \mathrm{m}^{3}\right), \mathrm{BAF}=$ degree of anisotropy, $\phi=$ fluence $\left(10^{25} \mathrm{n} / \mathrm{m}^{2}\right), \mathrm{T}=$ temperature -20 $\left({ }^{\circ} \mathrm{C}\right), \mathrm{E}_{01}=\mathrm{E}_{03}=25.5 \mathrm{GPa}$.

In PARFUME, the value for crystallite diameter size, $L_{c}$, is set equal to 30 Angstroms, resulting in the fourth factor of Equations (7-2) and (7-3) taking on a value equal to 1.

\subsubsection{Pyrocarbon (buffer layer)}

PyC exhibits isotropic characteristics at low density, i.e. densities less than $1.8 \mathrm{Mg} / \mathrm{m}^{3}$; this density corresponds to a porosity, $p$, of $20 \%$. In PARFUME, a density of $1 \mathrm{Mg} / \mathrm{m}^{3}$ is assumed for the buffer layer, which corresponds to a porosity of $56 \%$. The following relation is used to compute modulus of elasticity:

$E=5 \times 10^{6} \exp (-2.03 \cdot p)(1+0.23 \phi)(1+0.00015 T)$

where $E$ is in units of psi, and $\phi$ and $T$ are as above. 


\subsubsection{Silicon Carbide (SiC Layer)}

The elastic modulus for $\mathrm{SiC}$ is a function of temperature as shown in Table 7-1.

Table 7-1. SiC Modulus of Elasticity as a Function of Temperature

\begin{tabular}{c|c}
\hline $\mathrm{T}\left({ }^{\circ} \mathrm{C}\right)$ & $\mathrm{E}(\mathrm{MPa})$ \\
\hline 25 & $4.276 \times 10^{5}$ \\
940 & $3.752 \times 10^{5}$ \\
1215 & $3.400 \times 10^{5}$ \\
1600 & $1.979 \times 10^{5}$ \\
\hline
\end{tabular}

The elastic moduli at temperatures between those listed are determined by linearly interpolating between the values given.

\subsubsection{Poisson's Ratio}

\subsubsection{Pyrocarbons (IPyC and OPyC layers)}

Since the dependency of Poisson's ratio $(v)$ on the various factors which effect material properties is unknown, it is assumed that the value for $v$ is a constant 0.23 , which is the value prescribed in Reference 25 for isotropic pyrocarbon.

\subsubsection{Pyrocarbon (buffer layer)}

Since the buffer layer behaves as an isotropic material at low density, a value of 0.23 for Poisson's ratio is used in PARFUME.

\subsubsection{Silicon Carbide (SiC Layer)}

In accordance with recommendations of Reference 25, the Poisson's ratio for the $\mathrm{SiC}$ is set equal to 0.13 in PARFUME.

\subsubsection{Irradiation-induced Dimensional Change}

\subsubsection{Pyrocarbons (IPyC and OPyC layers)}

Incorporating the swelling strain physics requires some detailed interpolation to treat the dependence on temperature, density, and BAF. This process starts with fourth-order polynomials that define swelling 
strains as a function of fluence at given values of BAF (ranging from 1.0 to 1.3333) and temperature $\left(600^{\circ} \mathrm{C}, 1032^{\circ} \mathrm{C}\right.$, or $\left.1350^{\circ} \mathrm{C}\right)$. The general form of the polynomial is shown in Equation (7-5).

$\varepsilon_{i}=a_{1} \phi+a_{2} \phi^{2}+a_{3} \phi^{3}+a_{4} \phi^{4}$

where the subscript $i$ represents either the radial (r) or tangential ( $\mathrm{t}$ ) component of strain; the isotropic component of strain (i.e., $\varepsilon_{t}=\varepsilon_{r}$ ) is represented as $\varepsilon_{T}$. These polynomials are differentiated to give thirdorder polynomials for swelling strain rates. Polynomial coefficients are given in Table 7-2 for both the radial and tangential directions.

Table 7-2. Polynomial Coefficients for PyC with $\rho=1.96 \mathrm{Mg} / \mathrm{m}^{3}$

\begin{tabular}{c|c|c|c|c|c|c|c|c}
\hline $\mathrm{T}(\mathrm{C})$ & Component & $\mathrm{R}($ note 1$)$ & $\mathrm{BAF}_{\mathrm{o}}$ & $\mathrm{a}_{1}$ & $\mathrm{a}_{2}$ & $\mathrm{a}_{3}$ & $\mathrm{a}_{4}$ & $\mathrm{r}$ (note 2$)$ \\
\hline 1350 & radial & 0.61 & 1.2787 & 3.7162 & -2.7042 & 1.1799 & -0.13910 & 1.0000 \\
1350 & radial & 0.63 & 1.1746 & 1.2093 & -0.53861 & 0.43114 & -0.05590 & 1.000 \\
1350 & radial & 0.65 & 1.0769 & -0.89522 & 0.80331 & -0.09009 & 0.00467 & 0.999 \\
1350 & radial & 0.656 & 1.0488 & -1.4964 & 1.16621 & -0.30106 & 0.03475 & 0.998 \\
1350 & radial & 0.662 & 1.0212 & -1.5433 & 0.59804 & -0.09997 & 0.00978 & 0.999 \\
1350 & isotropic & $2 / 3$ & 1.000 & -1.4284 & -0.19563 & 0.18991 & -0.02591 & 0.998 \\
1350 & tangential & 0.67 & 1.0303 & -2.2468 & 0.48243 & -0.07687 & 0.00464 & 1.000 \\
1350 & tangential & 0.675 & 1.0769 & -2.8293 & 0.76088 & -0.22314 & 0.02431 & 1.000 \\
1350 & tangential & 0.68 & 1.125 & -3.2555 & 0.90423 & -0.33175 & 0.04329 & 0.999 \\
1350 & tangential & 0.69 & 1.2258 & -4.4478 & 1.6032 & -0.58683 & 0.07458 & 0.999 \\
1350 & tangential & 0.70 & 1.3333 & -5.6714 & 2.4192 & -0.86155 & 0.10668 & 0.999 \\
1032 & radial & 0.61 & 1.2787 & -0.45900 & 0.51172 & -0.03245 & -0.00142 & 0.949 \\
1032 & radial & 0.63 & 1.1746 & -1.1854 & 0.64995 & 0.01380 & -0.01284 & 0.988 \\
1032 & radial & 0.65 & 1.0769 & -1.8169 & 1.1085 & -0.23868 & 0.02484 & 0.997 \\
1032 & radial & 0.656 & 1.0488 & -2.0047 & 1.3038 & -0.3728 & 0.04538 & 1.000 \\
1032 & radial & 0.662 & 1.0212 & -2.0752 & 1.37845 & -0.48993 & 0.06602 & 1.000 \\
1032 & isotropic & $2 / 3$ & 1.000 & -1.5239 & 0.13048 & 0.06299 & -0.01072 & 1.000 \\
1032 & tangential & 0.67 & 1.0303 & -1.5759 & 0.09019 & 0.05306 & -0.00815 & 1.000 \\
1032 & tangential & 0.675 & 1.0769 & -1.3220 & -0.51928 & 0.27603 & -0.03465 & 1.000 \\
1032 & tangential & 0.68 & 1.125 & -1.1870 & -0.90635 & 0.41046 & -0.05067 & 1.000 \\
1032 & tangential & 0.69 & 1.2258 & -0.96963 & -1.5911 & 0.64689 & -0.07682 & 1.000 \\
1032 & tangential & 0.70 & 1.3333 & -0.81239 & -2.20760 & 0.88496 & -0.10457 & 0.999 \\
600 & radial & 0.61 & 1.2787 & 0.40265 & -0.16501 & 0.03676 & 0.00706 & 0.999 \\
600 & radial & 0.63 & 1.1746 & -0.15714 & -0.14889 & 0.07546 & -0.00293 & 0.985 \\
\hline & & & & & & & & \\
1309
\end{tabular}


Table 7-2. Polynomial Coefficients for PyC with $\rho=1.96 \mathrm{Mg} / \mathrm{m}^{3}$ (continued)

\begin{tabular}{c|c|c|c|c|c|c|c|c}
\hline $\mathrm{T}(\mathrm{C})$ & Component & $\mathrm{R}($ note 1) & $\mathrm{BAF}_{\mathrm{o}}$ & $\mathrm{a}_{1}$ & $\mathrm{a}_{2}$ & $\mathrm{a}_{3}$ & $\mathrm{a}_{4}$ & $\mathrm{r}$ (note 2) \\
\hline 600 & radial & 0.65 & 1.0769 & -0.78045 & -0.02975 & 0.06655 & -0.00626 & 0.994 \\
600 & radial & 0.656 & 1.0488 & -0.94333 & -0.03589 & 0.08184 & -0.00958 & 0.996 \\
600 & radial & 0.662 & 1.0212 & -1.1064 & -0.03128 & 0.09184 & -0.01220 & 0.998 \\
600 & isotropic & $2 / 3$ & 1.000 & -1.2408 & 0.00175 & 0.08533 & -0.01253 & 0.998 \\
600 & tangential & 0.67 & 1.0303 & -1.3855 & 0.05307 & 0.07620 & -0.01245 & 0.999 \\
600 & tangential & 0.675 & 1.0769 & -1.4679 & -0.02836 & 0.12139 & -0.01948 & 0.999 \\
600 & tangential & 0.68 & 1.125 & -1.6466 & 0.03928 & 0.10067 & -0.01764 & 0.999 \\
600 & tangential & 0.69 & 1.2258 & -1.8499 & -0.09358 & 0.18119 & -0.03036 & 0.999 \\
600 & tangential & 0.70 & 1.3333 & -2.1919 & 0.02675 & 0.15352 & -.02972 & 0.999 \\
\hline
\end{tabular}

Notes:

(1) $\mathrm{R}=$ orientation parameter giving a measure of anisotropy

(2) $r=$ statistical coefficient indicating closeness of curve fit to data

Isotropic swelling strain computed using Equation (7-5) is adjusted for other densities by applying a scaling factor, $u_{\rho}$, defined in Equation (7-6). The scaling factor is the ratio of the isotropic strain at a given density to the isotropic strain at a density of $1.96 \mathrm{Mg} / \mathrm{m}^{3}$. The values for $\varepsilon_{T}(\rho)$ are obtained from Table 73 .

$u_{\rho}=\varepsilon_{T}(\rho) / \varepsilon_{T}\left(\rho=1.96 \mathrm{Mg} / \mathrm{m}^{3}\right)$

Table 7-3. $\varepsilon_{T}$ at $1100{ }^{\circ} \mathrm{C}$ Irradiation Temperature to $3.7 \times 10^{25} \mathrm{n} / \mathrm{m}^{2}$

\begin{tabular}{c|c|c|c|c|c|c|c|c|c}
\hline$\rho, \mathrm{g} / \mathrm{cm}^{3}$ & 1.0 & 1.2 & 1.4 & 1.5 & 1.6 & 1.8 & 1.9 & 1.96 & 2.0 \\
$\varepsilon_{T}, \%$ & -16.15 & -13.11 & -9.98 & -8.93 & -6.97 & -4.42 & -3.41 & -2.75 & -2.33 \\
\hline
\end{tabular}

Adjustments for density at other BAF values are made by applying a scaling factor $v_{\rho}$ to the difference between the radial and tangential strain components; as defined in Equation (7-7), the scaling factor is the ratio of the strain difference at a given density to the strain difference at a density of $1.96 \mathrm{Mg} / \mathrm{m}^{3}$. The values for $\left(\varepsilon_{r}-\varepsilon_{t}\right)(\rho)$ are obtained from Table 7-4. 
$v_{\rho}=\left(\varepsilon_{r}-\varepsilon_{t}\right)(\rho) /\left(\varepsilon_{r}-\varepsilon_{t}\right)\left(\rho=1.96 \mathrm{Mg} / \mathrm{m}^{3}\right)$

Table 7-4. $\varepsilon_{r}-\varepsilon_{t}$ at $1100{ }^{\circ} \mathrm{C}$ Irradiation Temperature to $3.7 \times 10^{25} \mathrm{n} / \mathrm{m}^{2}$

\begin{tabular}{c|c|c|c|c|c|c|c|c|c}
\hline$\rho, \mathrm{g} / \mathrm{cm}^{3}$ & 1 & 1.2 & 1.4 & 1.5 & 1.6 & 1.8 & 1.9 & 1.96 & 2.0 \\
$\varepsilon_{r}-\varepsilon_{t}, \%$ & 0 & 0.5 & 1.1 & 1.65 & 2.45 & 6.305 & 7.9 & 9.6 & 11.1 \\
\hline
\end{tabular}

Using Equations (7-8) and (7-9), the general process used to compute strain is summarized below.

$\varepsilon_{T \rho}=u_{\rho} \varepsilon_{T}$

$\varepsilon_{r \rho}-\varepsilon_{t \rho}=v_{\rho}\left(\varepsilon_{r}-\varepsilon_{t}\right)$

As shown in Figure 7-1, which was extracted from Reference 25, the radial and tangential components of swelling strain at any density straddle the isotropic swelling strain at that density.

For the density of $1.96 \mathrm{Mg} / \mathrm{m}^{3}$, this relationship can be approximated as follows:

$\varepsilon_{T}=\varepsilon_{t}+k\left(\varepsilon_{r}-\varepsilon_{t}\right)$

where $\varepsilon_{r}>\varepsilon_{t}$. The quantity $k$ is determined by solving Equation (7-10) using the polynomial data for the standard density, $\rho=1.96 \mathrm{Mg} / \mathrm{m}^{3}$. The same value of $k$ is then used to make this interpolation at other densities. This results in the following expressions for the swelling strain rates at any density:

$\varepsilon_{r p}=u_{p} \varepsilon_{T}+v_{p}\left(\varepsilon_{r}-\varepsilon_{T}\right)$

$\varepsilon_{t p}=u_{p} \varepsilon_{T}+v_{p}\left(\varepsilon_{t}-\varepsilon_{T}\right)$

These density interpolations are made at the two temperature values that straddle the temperature under consideration. Then, the final swelling strain rates are determined by interpolating between these two temperature values. 


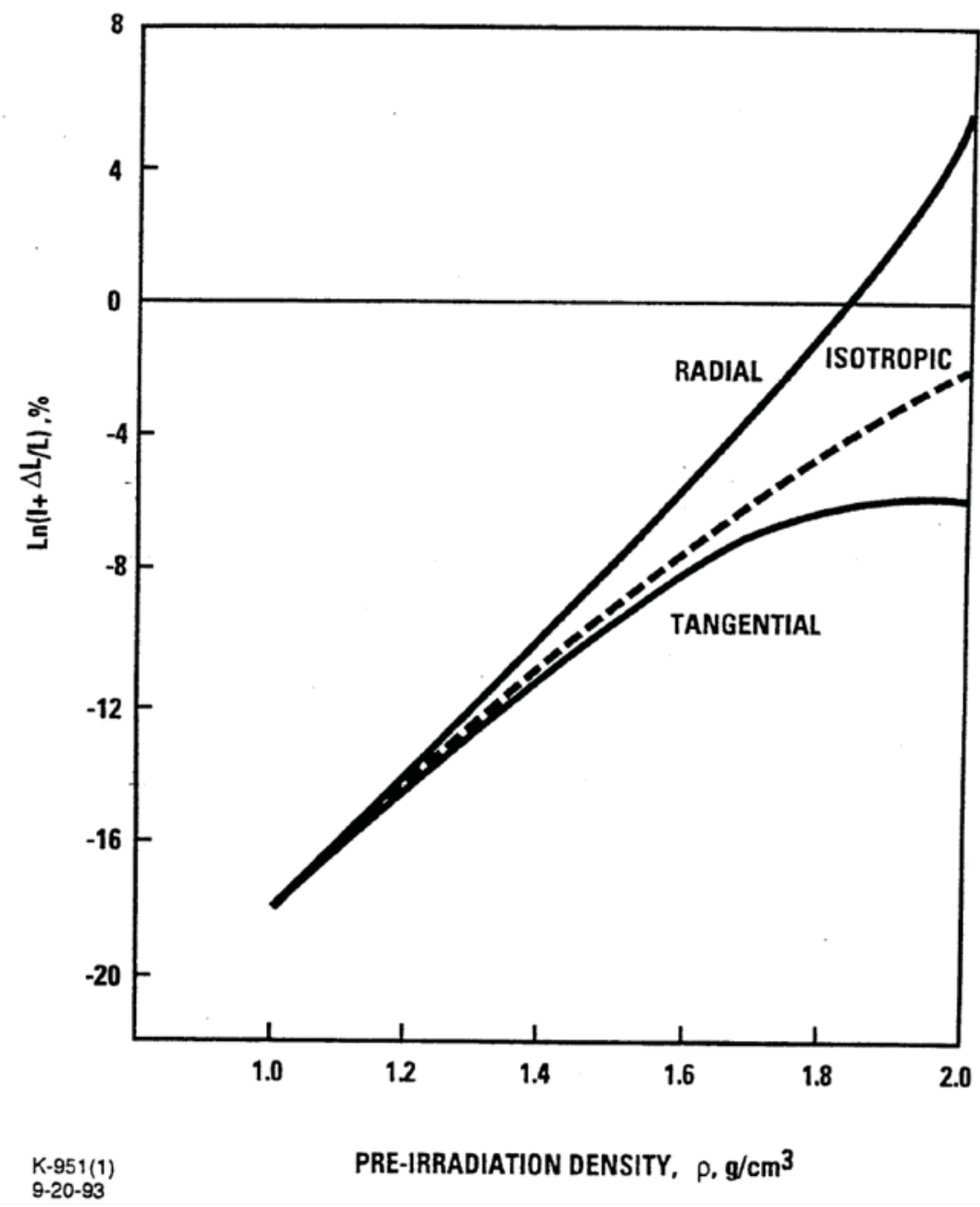

Figure 7-1. Irradiation Strain vs. Preirradiation Density for PyC irradiated at $1100{ }^{\circ} \mathrm{C}$ to $3.7 \times 10^{25} \mathrm{n} / \mathrm{m}^{2}$

Reference 25 presents a relationship between BAF and fluence, which indicates that BAF increases steadily with fluence. The relationship (shown in Table 7-5) is the ratio between BAF at fluence $\phi$ and $\mathrm{BAF}$ at zero fluence and is programmed into the PARFUME code.

Table 7-5. BAF as a function of fluence

\begin{tabular}{c|c|c|c|c|c|c|c|c}
\hline $\begin{array}{c}\phi, 10^{25} \mathrm{n} / \mathrm{m}^{2} \\
\text { BAF }\end{array}$ & 0 & 1 & 2 & 3 & 3.5 & 4 & 4.5 & $\geq 5$ \\
& 1.0000 & 1.0019 & 1.0114 & 1.0219 & 1.0786 & 1.0324 & 1.0362 & 1.0381 \\
\hline
\end{tabular}

\subsubsection{Pyrocarbon (buffer layer)}

Similar to the PyC layer, dimensional changes such as swelling are computed using Equation (7-5). The coefficients for the equation are obtained from Table 7-2. Since the buffer layer is assumed to behave 
as an isotropic material, the coefficients used correspond to $\mathrm{BAF}_{\mathrm{o}}=1$ associated with the temperatures of $1350{ }^{\circ} \mathrm{C}, 1032^{\circ} \mathrm{C}$, or $600^{\circ} \mathrm{C}$. Deformation at other temperatures is determined by interpolating between these temperatures.

\subsubsection{Silicon Carbide (SiC Layer)}

Irradiation-induced dimensional change for the $\mathrm{SiC}$ layer is assumed to be negligible in accordance with the recommendation of Reference 25 . This has historically not been included in performance models, though Reference 25 indicates that there can be situations where it is significant, such as "when either or both of the IPyC and OPyC peel away from the SiC layer." Irradiation-induced swelling of the SiC could be readily added to the PARFUME model using the methodology presented in Section 4 if an analysis of data, such as in References 25 and 44, indicate a need to do so.

\subsubsection{Weibull Strength Parameters}

The Weibull characteristic strength $\left(\sigma_{o}\right)$ for the PyC layers is a function of BAF, temperature, and fluence. The Weibull modulus for the IPyC is held constant throughout irradiation. Reference 25 indicates that the strength of the SiC may be a function of temperature, but that this dependence needs further study. The Weibull characteristic strength and modulus for the SiC are therefore currently held constant throughout irradiation.

\subsubsection{Pyrocarbons (IPyC and OPyC layers)}

The Weibull statistical strength theory is applied in the code according to the following formula:

$P_{f}=1-\exp \left[-\int_{V}\left(\sigma / \sigma_{0}\right)^{m} d V\right]$

It is noted that, since compressive stresses do not contribute to failure of a coating layer, only tensile stresses are included in the integral above.

In keeping with recommendations of Reference 25, the Weibull modulus for the pyrocarbon layers is not treated as a function of fluence or temperature. A value of 9.5 , which corresponds to a density of $1.9 \mathrm{Mg} / \mathrm{m}^{3}$, is assumed for the modulus.

The second Weibull parameter, the characteristic strength of the pyrocarbons, is a function of anisotropy and is determined from the following equation for room temperature:

$\sigma_{0}=154.46 B A F_{0}{ }^{2}-141.1 B A F_{0}$

where $B A F_{o}$ is the unirradiated value of $B A F$. The characteristic strength has units of MPa-m $\mathrm{m}^{3 / 9.5}$.

At other temperatures and non-zero fluence, Equation (7-14) is multiplied by the factor $\left(\mathrm{E}_{0 \mathrm{i}} / \mathrm{E}_{0}\right)^{1 / 2}$ as follows: 
$\left(\frac{E_{0 i}}{E_{0}}\right)^{1 / 2}=\left[\frac{(1+0.23 \phi)(1+0.00015 T)}{1+0.00015 T_{r}}\right]^{1 / 2}$

where

$$
\begin{aligned}
& \phi=\text { fluence }\left(10^{25} \mathrm{n} / \mathrm{m}^{2}\right) \\
& T=\text { temperature }-20\left({ }^{\circ} \mathrm{C}\right) \text { and } T_{r}=\text { room temperature }-20\left({ }^{\circ} \mathrm{C}\right)
\end{aligned}
$$

\subsubsection{Pyrocarbon (buffer layer)}

Currently, the evaluation of buffer layer failure is not included in PARFUME.

\subsubsection{Silicon Carbide (SiC Layer)}

The following Weibull parameters are used for the SiC layer in PARFUME:

$$
m=6 \text { and } \sigma_{o}=9.64 \mathrm{MPa}-\mathrm{m}^{3 / 6}
$$

\subsubsection{Irradiation-induced Creep}

\subsubsection{Pyrocarbons (IPyC and OPyC layers)}

Irradiation-induced steady state creep is treated as secondary creep with a coefficient that is a function of pyrocarbon density and irradiation temperature. Though transient creep could have an effect early in irradiation, the parameters needed to model it are not well established. Furthermore, a study of transient creep $^{46}$ showed it to have at most a nominal effect on particle stresses. The steady state creep coefficient is obtained from the following equation:

$K_{S}=[1+2.38(1.9-\rho)]\left(2.193 \times 10^{-4}-4.85 \times 10^{-7} T+4.0147 \times 10^{-10} T^{2}\right)$

where $T=$ temperature $\left({ }^{\circ} \mathrm{C}\right)$ and $K_{S}$ has units of $10^{-25}\left(\mathrm{MPa}-\mathrm{n} / \mathrm{m}^{2}\right)^{-1}$. Note that due to a lack of available data, $K_{S}$ is assumed to be independent of fast fluence. Note that the code provides the user with an input parameter called CREEPAMP, which is a factor applied to $K_{S}$ that can be used to amplify (or reduce) the creep coefficient if desired.

\subsubsection{Pyrocarbon (buffer layer)}

Using a buffer density equal to $1 \mathrm{Mg} / \mathrm{m}^{3}$, Equation (7-16) is used to compute the steady state creep coefficient for the buffer layer.

\subsubsection{Silicon Carbide (SiC Layer)}

Irradiation-induced creep in the SiC layer is not computed in PARFUME. 


\subsection{Thermal}

\subsubsection{Thermal Conductivity}

Functional relationships for the thermal conductivity for $\mathrm{PyC}$ and $\mathrm{UO}_{2}$ were obtained from Reference 47. The relationships are presented below. Due to the lack of published UCO material properties and respective correlations, functional relationships for $\mathrm{UCO}$ are assumed to be the same as $\mathrm{UO}_{2}$.

\subsubsection{Pyrocarbons (IPyC and OPyC layers)}

$\mathrm{k}=4 \mathrm{~W} / \mathrm{m} / \mathrm{K}$

\subsubsection{Pyrocarbon (buffer layer)}

$\mathrm{k}=0.5 \mathrm{~W} / \mathrm{m} / \mathrm{K}$

\subsubsection{3. $\mathrm{UO}_{2}$ Fuel}

$k(T)=0.0132 e^{1.88 E-3 T}+\frac{4040}{464+T}$ in $\mathrm{W} / \mathrm{m} / \mathrm{K}$ and $\mathrm{T}$ in ${ }^{\circ} \mathrm{C}$

for $T<1650{ }^{\circ} \mathrm{C}$

$k(T)=0.0132 e^{1.88 E-3 T}+1.9$

for $T \geq 1650{ }^{\circ} \mathrm{C}$

\subsubsection{SiC Layer}

$k(T)=\frac{17885}{T+273}+2$ in $\mathrm{W} / \mathrm{m} / \mathrm{K}$ and $\mathrm{T}$ in ${ }^{\circ} \mathrm{C}$

\subsubsection{Matrix}

The correlation used in PARFUME for the matrix thermal conductivity was obtained from Reference 48 where:

$k(T)=f($ unirradiated $) \cdot f($ fluence $) \cdot f($ packing $) \cdot f($ matrix density $)$

$f($ unirradiated $)=k_{100}\left[1-\alpha T^{\prime} e^{\delta T}\right]$

$f($ fluence $)=1-\gamma\left[1-e^{-\beta T}\right]-\varepsilon \Gamma$ 
$f($ packing $)=\frac{1-P}{1+\frac{P}{2}}$

$f($ matrix density $)=\frac{\text { matrix density }}{1.7 \mathrm{mg} / \mathrm{m}^{3}}$

and

$\mathrm{T}=$ temperature in ${ }^{\circ} \mathrm{C}$ and $T^{\prime}=\mathrm{T}-100{ }^{\circ} \mathrm{C}$

$\Gamma=\frac{\text { fast neutron fluence in } \mathrm{n} / \mathrm{m}^{2} \text { for } \mathrm{E}>0.18 \mathrm{MeV}}{1.52 \times 10^{25} \mathrm{n} / \mathrm{m}^{2}}$

$\gamma=0.940-0.604\left(\frac{T}{1000}\right)$

$\beta=2.960-1.955\left(\frac{T}{1000}\right)$

$\varepsilon=0.043\left(\frac{T}{1000}\right)-0.008\left(\frac{T}{1000}\right)^{8}$

$P=\frac{\text { (number particles per compact })(\text { volume per particle })}{\text { (volume per compact) }}$

Note that for A3-27 material (i.e., carbon) heat treated at $1800 \mathrm{C}$ :

$\mathrm{k}_{100}=47.4 \mathrm{~W} / \mathrm{mK}$

$\alpha=9.7556 \times 10^{-4}$

$\delta=-6.0360 \times 10^{-4}$

\subsubsection{Specific Heat Capacity}

\subsubsection{Pyrocarbons (IPyC and OPyC layers)}

The specific heat capacity for the IPyC and OPyC is not required in PARFUME since a quasi-steady approach is utilized in the heat transfer calculations for fuel particles.

\subsubsection{Pyrocarbon (buffer layer)}

The specific heat capacity for the buffer layer is not required in PARFUME since a quasi-steady approach is utilized in the heat transfer calculations for fuel particles. 


\subsubsection{Fuel}

The specific heat capacity for fuel is not required in PARFUME since a quasi-steady approach is utilized in the heat transfer calculations for fuel particles.

\subsubsection{Matrix}

The specific heat capacity correlation for the fuel element matrix, shown in Equation (7-24) for carbon brick was taken from Reference 49.

$c_{p}=1.75 \times 10^{6}\left(6.45+3.14 T-2.809 T^{2}+0.959 T^{3}\right)$

The units for specific heat capacity are $\mathrm{J} /\left(\mathrm{m}^{3} \mathrm{~K}\right)$ and

$$
T=\frac{{ }^{\circ} \mathrm{C}}{1000}
$$

The minimum allowed value for specific heat capacity is $3.54 \times 10^{-6} \mathrm{~J} /\left(\mathrm{m}^{3}-\mathrm{K}\right)$.

\subsubsection{Thermal Expansion}

\subsubsection{Pyrocarbons (IPyC and OPyC layers)}

The coefficients of thermal expansion in the radial and tangential directions for the pyrocarbons are functions of an orientation parameter $R$ as follows:

$\alpha_{r}=A\left(-37.5 R_{r}+30\right)$

$\alpha_{t}=A\left[36\left(R_{t}-1\right)^{2}+1\right]$

in units of $10^{-6} /{ }^{\circ} \mathrm{C}$, where $R_{t} \leq 2 / 3$ and $R_{r} \leq 2 / 3$, and the orientation parameters in the radial and tangential directions are related to the BAF as follows:

$R_{r}=\frac{2}{2+B A F}$

$R_{t}=1-\frac{R_{r}}{2}$

and $A$ is a temperature adjustment factor given by

$A=1+0.11 \frac{(T-400)}{700}$

and $T$ is the temperature of the pyrocarbon layer in ${ }^{\circ} \mathrm{C}$. 


\subsubsection{Pyrocarbon (buffer layer)}

The buffer layer utilizes the equations presented in 7.2.3.1. Due to its isotropic behavior, $R_{r}=R_{t}=R=2 / 3$.

\subsubsection{Silicon Carbide (SiC Layer)}

The thermal expansivity of $\mathrm{SiC}$ has a mean value of $4.9 \times 10^{-6}$ per ${ }^{\circ} \mathrm{C}$ for a temperature range between $22{ }^{\circ} \mathrm{C}$ and $1000{ }^{\circ} \mathrm{C}$; furthermore, it is assumed that thermal expansivity remains constant between $900{ }^{\circ} \mathrm{C}$ and $1300^{\circ} \mathrm{C}$. Therefore, a constant value of $4.9 \times 10^{-6}$ per ${ }^{\circ} \mathrm{C}$ is used in PARFUME. Note that the presence of free carbon in the $\mathrm{SiC}$ layer could reduce the thermal expansivity by $40 \%$.

\subsection{Diffusion Coefficients}

Currently PARFUME uses models available in TMAP (The Tritium Migration Analysis Program), a finite difference based code, to predict fission product diffusion. TMAP incorporates a one-dimensional diffusion capability that predicts the thermal response of structures (i.e., fuel particles), and solves equations for gas movement through surfaces (layer interfaces) and in bulk materials (i.e., layers). In order to predict the migration of fission products, diffusion coefficients must be determined.

Diffusion coefficients for all nuclides considered within the PARFUME FP transport models are listed in Table 7-6. ${ }^{46}$ The corresponding diffusivities can be calculated using these coefficients in an Arrheniustype equation given by

$$
D(T)=\sum_{i} D_{0, i} \exp \left(-\frac{Q_{i}}{R T}\right)
$$

where

$D_{0}=$ the pre-exponential factor $\left(\mathrm{m}^{2} / \mathrm{s}\right)$,

$Q=$ the activation energy $(\mathrm{J} / \mathrm{mol})$,

$R=$ the gas constant $(8.3142 \mathrm{~J} / \mathrm{mol} \mathrm{K})$, and

$T=$ temperature $(\mathrm{K})$. 
Table 7-6. PARFUME diffusion coefficients.

\begin{tabular}{|c|c|c|c|c|c|}
\hline Material & Nuclide & $\mathrm{D}_{0,1}\left(\mathrm{~m}^{2} / \mathrm{s}\right)$ & $\mathrm{Q}_{1}(\mathrm{~kJ} / \mathrm{mol})$ & $\mathrm{D}_{0,2}\left(\mathrm{~m}^{2} / \mathrm{s}\right)$ & $\mathrm{Q}_{2}(\mathrm{~kJ} / \mathrm{mol})$ \\
\hline \multirow{5}{*}{$\begin{array}{c}\text { fuel kernel } \\
\text { (either } \mathrm{UO}_{2} \text { or } \mathrm{UCO} \text { ) }\end{array}$} & $\mathrm{Ag}$ & $6.7 e-9$ & 165 & & \\
\hline & $\mathrm{Cs}$ & $5.6 e-8$ & 209 & $5.2 \mathrm{e}-4$ & 362 \\
\hline & $\begin{array}{c}\mathrm{Kr} \\
\text { (normal operation) }\end{array}$ & $1.3 e-12$ & 126 & & \\
\hline & $\begin{array}{c}\mathrm{Kr} \\
\text { (accident conditions) }\end{array}$ & $8.8 \mathrm{e}-15$ & 54 & $6.0 \mathrm{e}-1$ & 480 \\
\hline & $\mathrm{Sr}$ & $2.2 \mathrm{e}-3$ & 488 & & \\
\hline \multirow{4}{*}{ Buffer } & $\mathrm{Ag}$ & $1.0 \mathrm{e}-8$ & 0 & & \\
\hline & Cs & $1.0 \mathrm{e}-8$ & 0 & & \\
\hline & $\mathrm{Kr}$ & $1.0 \mathrm{e}-8$ & 0 & & \\
\hline & $\mathrm{Sr}$ & $1.0 \mathrm{e}-8$ & 0 & & \\
\hline \multirow{4}{*}{ PyC } & $\mathrm{Ag}$ & $5.3 e-9$ & 154 & & \\
\hline & Cs & $6.3 e-8$ & 222 & & \\
\hline & $\mathrm{Kr}$ & $2.9 \mathrm{e}-8$ & 291 & $2.0 \mathrm{e} 5$ & 923 \\
\hline & $\mathrm{Sr}$ & $2.3 e-6$ & 197 & & \\
\hline \multirow{5}{*}{$\mathrm{SiC}$} & $\mathrm{Ag}$ & $3.6 \mathrm{e}-9$ & 215 & & \\
\hline & $\mathrm{Cs}^{\mathrm{a}}$ & $5.5 e-14 * e^{\Gamma / 4.5}$ & 125 & $1.6 \mathrm{E}-2$ & 514 \\
\hline & $\begin{array}{c}\mathrm{Kr} \\
(\text { temperature }<1626 \mathrm{~K})\end{array}$ & $8.6 e-10$ & 326 & & \\
\hline & $\begin{array}{c}\mathrm{Kr} \\
\text { (temperature }>1626 \mathrm{~K} \text { ) }\end{array}$ & $3.7 \mathrm{e} 1$ & 657 & & \\
\hline & $\mathrm{Sr}$ & $1.2 \mathrm{e}-9$ & 205 & $1.8 \mathrm{e} 6$ & 791 \\
\hline \multirow{4}{*}{$\begin{array}{c}\text { matrix graphite } \\
\text { (either A3-3 or A3-27) }\end{array}$} & $\mathrm{Ag}$ & $1.6 \mathrm{e} 0$ & 258 & & \\
\hline & Cs & $3.6 \mathrm{e}-4$ & 189 & & \\
\hline & $\mathrm{Kr}$ & $6.0 \mathrm{e}-6$ & 0 & & \\
\hline & $\mathrm{Sr}$ & $1.0 \mathrm{e}-2$ & 303 & & \\
\hline
\end{tabular}

a. Note that $\Gamma=$ fast neutron fluence $\left(\mathrm{e} 25 \mathrm{n} / \mathrm{m}^{2}, \mathrm{E}>0.18 \mathrm{MeV}\right)$. 


\section{REFERENCES}

1. H.S. Carslaw and J.C. Jaeger, Conduction of Heat in Solids, Second Edition, Oxford University, 1959.

2. $\quad$ "SCDAP/RELAP5/MOD3.2 Code Manual, MATPRO - A Library of Materials Properties for LightWater-Reactor Accident Analysis," NUREG/CR-6150, Vol.4, Rev. 1, INEL-96/0422, July 1998.

3. G. K. Miller and R. G. Bennett, Analytical Solution for Stresses in TRISO-coated Particles, J. Nucl. Mat., 206, 1993, p. 35.

4. G. K. Miller, Updated Solution for Stresses and Displacements in TRISO-coated Fuel Particles, J. Nucl. Mat., 374, 2008, p. 129.

5. J. L. Kaae, A Mathematical Model for Calculating Stresses win a Four-Layer Carbon-Silicon-Carbide-Coated Fuel Particle, J. Nucl. Mat. 32, 1969, p. 322.

6. H. Walther, On Mathematical Models for calculating the Mechanical Behavior of Coated Fuel Particles, Nucl. Eng. Design, 18, 1971, p. 11.

7. K. Bongartz, Status of the Fuel Stress and Failure Rate Calculations at KFA, JUL-1686, KFA Julich, $\mathrm{GmbH}, 1980$.

8. J. L. Kaae, Behavior of Pyrolytic Carbons Irradiated Under Mechanical Restraint, Carbon 12, 1974, p. 577.

9. I. S. Sokolnikoff, Mathematical Theory of Elasticity, 2nd ed., McGraw-Hill, New York, 1956, p. 84.

10. G. K. Miller, Stresses in a Spherical Pressure Vessel Undergoing Creep and Dimensional Changes, Int. J. of Solids and Struct., 32, 14, 1995, p. 2077.

11. K.O. Redlich and J.N.S. Kwong, On the Thermodynamics of Solutions. V. An Equation of State. Fugacities of Gaseous Solutions, Chem. Rev., 44, 1949, p. 233.

12. R.C. Reid, J.M. Prausnitz and B.E. Poling, The Properties of Gases and Liquids, Fourth Ed., McGraw-Hill, 1987.

13. U. Littmark and J. F. Ziegler, Handbook of Range Distributions for Energetic Ions in All Elements, Pergamon Press, 1980.

14. Booth, A Method of Calculating Fission Gas Release from UO2 Fuel and Its Implication to the X-2-f Loop Test, Atomic Energy of Canada Limited Report - 496, 1957.

15. J.T.Turnbull et al., The Diffusion Coefficient of Gaseous and Volatile Species During the Irradiation of Uranium Dioxide, J. Nucl. Mater., 107, 1982, p. 168.

16. W.J. Kovacs, K. Bongartz and D. T. Goodin, High-Temperature Gas-Cooled Reactor Fuel Pressure Vessel Performance Models, Nuc. Tech., 68, 1985, p. 344. 
17. E.Proksch, A Strigl, and H. Nabielek, Production of carbon monoxide during burn-up of $\mathrm{UO}_{2}$ kerneled from HTR fuel particles, J. Nucl. Mat., 107, 1982, p.280.

18. HSC Chemistry User's Guide, Version 5.0, Outokumpu Research Oy, Finland, 2002.

19. Moore, B.G. Schnitzler, C.A. Wemple, R.S. Babcock and D.E. Wessol, MOCUP: MCNP-ORIGEN2 Coupled Utility Program, INEL-95/0523, 1995.

20. J.F. Briesmeister, et al., MCNP - A General Monte Carlo N-Particle Transport Code, Los Alamos National Laboratory, Los Alamos, NM, 1997.

21. G. Croff, A User's Manual For The ORIGEN2 Computer Code, Oak Ridge National Laboratory, Oak Ridge, TN, 1980.

22. ABAQUS User's Manual, Version 6.7, 2007.

23. R.C. Martin, Compilation of Fuel Performance and Fission Product Transport Models and Database for MHTGR Design, ORNL/NPR-91/6, October 1993.

24. G. K. Miller et al., Statistical Approach and Benchmarking for Modeling of Multi-dimensional Behavior in TRISO-coated Fuel Particles, J. Nucl. Mat., 317, 2003, p. 69.

25. CEGA Corporation, NP-MHTGR Material Models of Pyrocarbon and Pyrolytic Silicon Carbide, CEGA-002820, Rev. 1, July 1993.

26. N.N. Nemeth, J.M Mandershield, J.P. Gyekenyesi, Ceramics Analysis and Reliability Evaluation of Structures (CARES) User's and Programmer's Manual, NASA Technical Paper 2916, 1989.

27. A.E. Green, A.J. Bourne, Reliability Technology, John Wiley and Sons, New York, 1972, p.244.

28. G. K. Miller et al., Consideration of the Effects on Fuel Particle Behavior from Shrinkage Cracks in the Inner Pyrocarbon Layer, J. Nucl. Mat., 295, 2001, p.205.

29. G. K. Miller, D. A. Petti, J. T. Maki, Development of an Integrated Performance Model for TRISOcoated Gas Reactor Particle Fuel, $1^{\text {st }}$ International Topical Meeting on High Temperature Reactor Technology (HTR), Petten, The Netherlands, April 2002.

30. G. K. Miller, D. A. Petti, J. T. Maki, Consideration of the Effects of Partial Debonding of the IPyC and Particle Asphericity on TRISO-coated Fuel Behavior, J. Nucl. Mat., 334, 2004, p. 79.

31. G. K. Miller, D. A. Petti, J. T. Maki, D. L. Knudson, An Evaluation of the Effects of SiC Layer Thinning on Failure of TRISO-coated Fuel Particles, J. Nucl. Mat., in publication.uring the Irradiation of Uranium Dioxide.

32. J.W. Ketterer and B.F. Myers, Capsule HRB-16 Postirradiation Examination Report, HTGR-85-053, GA-908012 N/c, September 1985.

33. Projektleitung Hochtemperaturreaktor-Brennstoffkreislauf (HBK), Projektbericht 1984, 1984. 
34. T.B. Lindemer, R.L. Pearson, Kernal Migration for HTGR Fuels from the System Th-U-Pu-C-O-N, Journal of the American Ceramic Society, 60, 1977, p. 5.

35. R.J. Lauf, T.B. Lindemer and R.L. Pearson, Out-of-Reactor Studies of Fission Product - Silicon Carbide Interactions in HTGR Fuel Particles, J. Nuc. Mater. 120, 1984, pp. 6-30.

36. K. Minato, et al., Fission Product Palladium - Silicon Carbide Interaction in HTGR Fuel Particles, $J$. Nuc. Mater. 172, 1990, p. 184.

37. F. Montgomery, Fission - Product SiC Reaction in HTGR Fuel, GA-905837/1, July 1981.

38. N. Tiegs, Fission Product Pd-SiC Interaction in Irradiated Coated - Particle Fuels, Nuc. Tech., 57, 1982, p. 389.

39. A.H. Bourker and G.J.Lieberman, Engineering Statistics, Prentice-Hall, Inc., 1965.

40. Longhurst, G. R., D. F. Holland, J. L. Jones, and B. J. Merrill, “TMAP4 User’s Manual,” EGG-FSP10315, Idaho National Laboratory, June 1992.

41. Longhurst, G. R., S. L. Harms, E. S. Marwil, and B. G. Miller, "Verification and Validation of TMAP4,” EGG-FSP-10347, Rev. 01, Idaho National Laboratory, March 1994.

42. D.R. Olander, Fundamental Aspects of Nuclear Reactor Fuel Elements, ERDA, TID-26711-P1, 1976.

43. H. Nabielek, e-mail communication, November 13, 2002, discusses unpublished document: H. Nabielek, Partikeln und Brennelemente für den HTR, KFA-HTA, January 24, 1991.

44. L.L. Snead, T. Nozawa, Y. Katoh, T. S. Byun, S. Kondo, and D. Petti. "Handbook of SiC Properties for Fuel Performance Modeling.” J. Nuclear Materials. 371, 2007, pp. 329-337.

45. K. Röllig, Release of Noble Gases and Halogens from the PBMR Core, Westinghouse Reaktor GmbH, GBRA 052 336, 2001.

46. G.K. Miller, Considerations of Irradiation-Induced Transient Creep in Fuel Particle Modeling, J. of Nucl. Sci. and Tech., 32, 10, Oct 1995, p. 989.

47. H. Nabielek, K. Fukuda, K. Minato, T. Ogawa, "Calculation of Particle Temperatures in NSRR Tests,” JAERI, unpublished draft from March 13, 1992.

48. R. Gontard and H. Nabielek, "Performance Evaluation of Modern HTR TRISO Fuels," HTA-IB05/90, Julich, July 31, 1990.

49. H. Niessen and S. Ball "Heat Transport and Afterheat Removal for Gas Cooled Reactors Under Accident Conditions,” IAEA-TECDOC-1163, International Atomic Energy Agency.

50. IAEA, "Fuel Performance and Fission Product Behavior in Gas Cooled Reactors," IAEA-TECDOC978, International Atomic Energy Agency, November 1997. 


\section{Appendix A}

Equations for quantities contained in the derivations of Section 4 are:

$K_{1}=-\frac{2 r^{3} r_{a}{ }^{3}(1-2 \mu)+r_{a}{ }^{3} r_{b}{ }^{3}(1+\mu)}{2 E r^{2}\left(r_{b}{ }^{3}-r_{a}{ }^{3}\right)}$

$K_{2}=\frac{2 r^{3} r_{b}{ }^{3}(1-2 \mu)+r_{a}{ }^{3} r_{b}^{3}(1+\mu)}{2 E r^{2}\left(r_{b}{ }^{3}-r_{a}{ }^{3}\right)}$

$K_{3}=-\frac{(1+v) r_{a}{ }^{3} r_{b}{ }^{3}+2(1-2 v) r^{3} r_{a}{ }^{3}}{2 r^{2}\left(r_{b}{ }^{3}-r_{a}{ }^{3}\right)}$

$K_{4}=\frac{(1+v) r_{a}{ }^{3} r_{b}^{3}+2(1-2 v) r^{3} r_{b}^{3}}{2 r^{2}\left(r_{b}^{3}-r_{a}^{3}\right)}$

A-4

$K_{5}=\frac{r_{a}{ }^{3} r_{b}{ }^{3} \ln \frac{r_{a}}{r_{b}}}{r^{2}\left(r_{b}{ }^{3}-r_{a}{ }^{3}\right)}+\frac{r}{3}$

$K_{6}=-\frac{r_{a}^{3} r_{b}^{3} \ln \frac{r_{a}}{r_{b}}}{r^{2}\left(r_{b}^{3}-r_{a}^{3}\right)}+\frac{2 r}{3}$

A-6

$K_{7}=\frac{2(v-\mu)}{3 E(v-1)}\left[\frac{r_{b}{ }^{3}\left(r^{3}-r_{a}{ }^{3}\right) \ln r_{b}-r_{a}{ }^{3}\left(r^{3}-r_{b}{ }^{3}\right) \ln r_{a}}{r^{2}\left(r_{b}{ }^{3}-r_{a}{ }^{3}\right)}-r \ln r\right]$

$Z=b_{2} c_{1}-\left(c_{2}-d_{1}\right)\left(b_{1}-a_{2}\right)$

A-8 
$B_{1}=\frac{a_{4} c_{I} c_{1}}{Z}$

$B_{2}=-\frac{d_{3} c_{O}\left(b_{1}-a_{2}\right)}{Z}$

$B_{3}=-\frac{a_{4} c_{I}\left(c_{2}-d_{1}\right)}{Z}$

$B_{4}=\frac{d_{3} c_{O} b_{2}}{Z}$

$x_{0}=\frac{1}{Z}\left\{\left[-c_{1} r_{3}+\left(b_{1}-a_{2}\right) r_{4}\right] \overline{\alpha_{S}} \frac{\Delta T_{S}}{\Delta t}+a_{1} c_{1} \frac{\Delta p}{\Delta t}-d_{2}\left(b_{1}-a_{2}\right) \frac{\Delta q}{\Delta t}+c_{1} a_{3} c_{I}\left(p_{n-1}-\frac{\Delta p}{\Delta t} t_{n-1}\right)\right.$

$-d_{4}\left(b_{1}-a_{2}\right) c_{O}\left(q_{n-1}-\frac{\Delta q}{\Delta t} t_{n-1}\right)+a_{5} c_{1}\left(\overline{S_{r I}}+\overline{\alpha_{r I}} \frac{\Delta T_{I}}{\Delta t}\right)+a_{6} c_{1}\left(\overline{S_{t I}}+\overline{\alpha_{t I}} \frac{\Delta T_{I}}{\Delta t}\right)$

$\left.-d_{5}\left(b_{1}-a_{2}\right)\left(\overline{S_{r O}}+\overline{\alpha_{r O}} \frac{\Delta T_{O}}{\Delta t}\right)-d_{6}\left(b_{1}-a_{2}\right)\left(\overline{S_{t O}}+\overline{\alpha_{t O}} \frac{\Delta T_{O}}{\Delta t}\right)\right\}$

$x_{1}=\frac{1}{Z}\left[c_{1} a_{3} c_{I} \frac{\Delta p}{\Delta t}-d_{4}\left(b_{1}-a_{2}\right) c_{O} \frac{\Delta q}{\Delta t}\right]$

A-14

$y_{0}=-\frac{1}{Z}\left\{\left[b_{2} r_{4}-r_{3}\left(c_{2}-d_{1}\right)\right] \overline{\alpha_{S}} \frac{\Delta T_{S}}{\Delta t}+a_{1}\left(c_{2}-d_{1}\right) \frac{\Delta p}{\Delta t}-b_{2} d_{2} \frac{\Delta q}{\Delta t}+a_{3}\left(c_{2}-d_{1}\right) c_{I}\left(p_{n-1}-\frac{\Delta p}{\Delta t} t_{n-1}\right)\right.$

$-b_{2} d_{4} c_{O}\left(q_{n-1}-\frac{\Delta q}{\Delta t} t_{n-1}\right)+a_{5}\left(c_{2}-d_{1}\right)\left(\overline{S_{r I}}+\overline{\alpha_{r I}} \frac{\Delta T_{I}}{\Delta t}\right)+a_{6}\left(c_{2}-d_{1}\right)\left(\overline{S_{t I}}+\overline{\alpha_{t I}} \frac{\Delta T_{I}}{\Delta t}\right)$

$\left.-b_{2} d_{5}\left(\overline{S_{r O}}+\overline{\alpha_{r O}} \frac{\Delta T_{O}}{\Delta t}\right)-b_{2} d_{6}\left(\overline{S_{t O}}+\overline{\alpha_{t O}} \frac{\Delta T_{O}}{\Delta t}\right)\right\}$ 
$y_{1}=-\frac{1}{Z}\left[a_{3}\left(c_{2}-d_{1}\right) c_{I} \frac{\Delta p}{\Delta t}-b_{2} d_{4} c_{O} \frac{\Delta q}{\Delta t}\right]$

A-16

$m_{1}, m_{2}=\frac{1}{2}\left\{B_{2}+B_{3} \pm \sqrt{\left(B_{2}+B_{3}\right)^{2}-4\left(B_{2} B_{3}-B_{1} B_{4}\right)}\right\}$

$v_{1}=\frac{B_{1} y_{1}-B_{3} x_{1}}{B_{2} B_{3}-B_{1} B_{4}}$

A-18

$v_{0}=\frac{\left(B_{2}+B_{3}\right) v_{1}+x_{1}-B_{3} x_{0}+B_{1} y_{0}}{B_{2} B_{3}-B_{1} B_{4}}$

$w_{0}=\frac{1}{B_{1}}\left(v_{1}-B_{2} v_{0}-x_{0}\right)$

$w_{1}=-\frac{B_{2} v_{1}+x_{1}}{B_{1}}$

$x_{0}{ }^{\prime}=\frac{1}{c_{2}-d_{1}}\left[-\left(c_{3}+c_{4}\right) \overline{\alpha_{S}} \frac{\Delta T_{S}}{\Delta t}+d_{2} \frac{\Delta q}{\Delta t}+d_{4} c_{O}\left(q-\frac{\Delta q}{\Delta t} t_{n-1}\right)+d_{5}\left(\overline{S_{r O}}+\alpha_{r O} \frac{\Delta T_{O}}{\Delta t}\right)\right.$

$\left.+d_{6}\left(\overline{S_{t O}}+\overline{\alpha_{t O}} \frac{\Delta T_{O}}{\Delta t}\right)-c_{1} \frac{\Delta p}{\Delta t}\right]$

$x_{1}{ }^{\prime}=\frac{1}{c_{2}-d_{1}} d_{4} c_{O} \frac{\Delta q}{\Delta t}$ 


$$
\begin{aligned}
& y_{0}^{\prime}=\frac{1}{b_{1}-a_{2}}\left[-\left(b_{3}+b_{4}\right) \overline{\alpha_{S}} \frac{\Delta T_{S}}{\Delta t}+a_{1} \frac{\Delta p}{\Delta t}+a_{3} c_{I}\left(p-\frac{\Delta p}{\Delta t} t_{n-1}\right)+a_{5}\left(\overline{S_{r I}}+\overline{\alpha_{r I}} \frac{\Delta T_{I}}{\Delta t}\right)\right. \\
& \left.+a_{6}\left(\overline{S_{t I}}+\overline{\alpha_{t I}} \frac{\Delta T_{I}}{\Delta t}\right)-b_{2} \frac{\Delta q}{\Delta t}\right] \\
& y_{1}^{\prime}=\frac{1}{b_{1}-a_{2}} a_{3} c_{I} \frac{\Delta p}{\Delta t} \\
& m_{1}^{\prime}=\frac{d_{3} c_{O}}{c_{2}-d_{1}} \\
& D_{2}^{\prime}=\left[\sigma_{r I}\left(t_{n-1}\right)+\frac{y_{0}{ }^{\prime}+y_{1}{ }^{\prime}}{m_{2}{ }^{\prime}}+\frac{y_{1}{ }^{\prime}}{m_{2}{ }^{\prime}} t_{n-1}\right] e^{-m_{2} t_{n-1}} \\
& m_{2}{ }^{\prime}=\frac{a_{4} c_{I}}{b_{1}-a_{2}} \\
& D_{1}^{\prime}=\left[\sigma_{r O}\left(t_{n-1}\right)+\frac{x_{0}{ }^{\prime}+x_{1}{ }^{\prime}}{m_{1}{ }^{\prime}}+\frac{x_{1}{ }^{\prime}}{m_{1}{ }^{\prime}} t_{n-1}\right] e^{-m_{1}{ }^{\prime} t_{n-1}} \\
& { }^{\prime}
\end{aligned}
$$

Thermal expansion in the $\mathrm{SiC}$ layer, represented by $\alpha_{S} \Delta T_{S}$, is assumed to be isotropic in this Appendix. 